\title{
Infection by string links and new structure in the knot concordance group
}

\author{
JOHN R BURKE
}

This paper highlights the importance of string link concordance in the understanding of knot concordance in general. The results of this paper show that there are infinitely many nontrivial knots in the groups $G_{n}$ of $n$-solvable knots modulo $n .5$-solvable knots, for $n$ greater than or equal to 2 , which are not concordant to any knot that is obtained by two or more iterated infections of an Arf invariant zero knot by knots. This latter class accounts for nearly all previously known examples of knots in $G_{n}, n$ greater than or equal to 2 .

In this paper we will generalize the concept of when a rational Laurent polynomial is strongly coprime to another, first introduced by Cochran, Harvey and Leidy, to include multivariable polynomials. We also prove the existence of multivariable polynomials which are strongly coprime to all single variable Laurent polynomials. From this definition of coprimality we define the derived series localized at $\mathcal{M}$ for a given sequence of multivariable polynomials $\mathcal{M}$. From such series we obtain refinements of the $n$-solvable filtration. The operation of infection by a string link is then used to demonstrate that for particular $\mathcal{M}$, certain quotients of successive terms of these refined filtrations have infinite rank.

57M25; 20J05

\section{Introduction}

A (classical) knot is a smooth embedding, $K: S^{1} \rightarrow S^{3}$ of an oriented circle into $S^{3}$. We say that two knots, $K_{0}$ and $K_{1}$, are equivalent if they are ambient isotopic. A (classical) link is defined to be a smooth embedding of $n$ disjoint oriented circles in $S^{3}$ with equivalence defined via ambient isotopy as above. We will refer to the set of knots up to ambient isotopy as $\mathcal{K}$. Under the connected sum operation, $\mathcal{K}$ forms a monoid with the unknot as the identity element. Unfortunately, there are no inverses under connected sum.

Two knots, $K_{0}: S^{1} \hookrightarrow S^{3} \cong S^{3} \times 0$ and $K_{1}: S^{1} \hookrightarrow S^{3} \cong S^{3} \times 1$, are said to be concordant if there exists a proper smooth embedding of an annulus into $S^{3} \times I$ that 


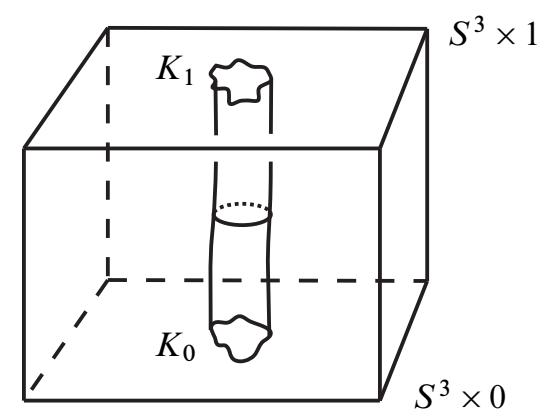

Figure 1: A schematic representation of a concordance cylinder

restricts to the knots on $S^{3} \times\{0,1\}$. A schematic representation of an embedded annulus in $S^{3} \times I$ is shown in Figure 1 .

Concordance is in fact an equivalence relation. We denote the set of concordance classes as $\mathcal{C}$. Concordance is a weaker relation than knot equivalence. Therefore, there is a well-defined surjection from $\mathcal{K}$ to $\mathcal{C}$. It can be shown that the connected sum operation is well defined on the concordance classes. It is known that the connected sum of any knot, $K$, with the reverse of its mirror image, $r \bar{K}$, is concordant to the unknot. (Here $r \bar{K}$ is obtained from $K$ by reversing the orientation of the knot along with changing all over-crossings to under-crossings.) Therefore, the class of $r \bar{K}$ is the inverse of the class represented by $K$. Hence, the set of concordance classes of knots forms an abelian group under the connected sum operation. We will also refer to this group as $\mathcal{C}$.

A slice knot in $S^{3}=\partial B^{4}$ is the boundary of a proper smooth embedding of a 2-disk in $B^{4}$. A knot is a slice knot if and only if it is concordant to the unknot. Therefore, the set of slice knots is the trivial class in $\mathcal{C}$. We can thus reinterpret concordance in the following way: Two knots, $K_{1}$ and $K_{2}$, are concordant if the connected sum of $K_{1}$ with $r \bar{K}_{2}$ is a slice knot.

The equivalence relation of concordance was first developed by Fox, Kervaire and Milnor in the 1960s while studying isolated singularities of 2-spheres in 4-manifolds [14; 17]. Certain concordance problems are known to be equivalent to whether higherdimensional surgery techniques are applicable to topological 4-manifolds. Therefore, being able to determine whether a knot is slice or not is a central problem in efforts toward the classification of 4-manifolds.

In the late 1960s Levine [22] defined an epimorphism from $\mathcal{C}$ onto $\mathbb{Z}^{\infty} \oplus \mathbb{Z}_{2}^{\infty} \oplus \mathbb{Z}_{4}^{\infty}$. In the early 1970s, Casson and Gordon [1; 2] showed that the kernel of this map 
is nontrivial. Using the higher-order signature invariants developed by Casson and Gordon, Gilmer constructed examples of knots in the kernel of Levine's epimorphism resulting from satellite operations on slice knots (see [2, Appendix]). This work was later expanded on by B Jiang [16] to show that the kernel, in fact, has infinite rank.

In 1997, Cochran, Orr and Teichner [11] defined the $n$-solvable filtration of $\mathcal{C}$ :

$$
\cdots \subseteq \mathcal{F}_{n .5} \subseteq \mathcal{F}_{n} \subseteq \cdots \subseteq \mathcal{F}_{1} \subseteq \mathcal{F}_{0.5} \subseteq \mathcal{F}_{0} \subseteq \mathcal{C}
$$

This filtration is important due to its strong connection to the work of Casson and Freedman on the topological classification problem for 4-manifolds. Also, the filtration encapsulates the classical concordance invariants in the lowest terms with the previously mentioned kernel being composed with all knots in $\mathcal{F}_{0.5}$. Thus, the complexity of the $n$-solvable filtration is related to the complexity of the kernel of Levine's epimorphism. We are able to study the complexity of $\mathcal{C}$ by examining the structure of the graded abelian groups $\mathcal{F}_{n} / \mathcal{F}_{n .5}$ for $n \in \mathbb{N}$. (Almost nothing is known about the other "half" of the filtration, $\mathcal{F}_{n .5} / \mathcal{F}_{n+1}$.)

The filtration has been shown to be nontrivial. In [12], Cochran, Orr and Teichner demonstrated that $\mathcal{F}_{2} / \mathcal{F}_{2.5}$ has infinite rank. It was then shown by Cochran and Teichner [13] that each of the groups $\mathcal{F}_{n} / \mathcal{F}_{n .5}$ has rank at least 1 . This work culminated with the work of Cochran, Harvey and Leidy [9], wherein it was shown that, for all $n \in \mathbb{N}$, the group $\mathcal{F}_{n} / \mathcal{F}_{n .5}$ has infinite rank.

In the 1960s and 1970s, Milnor, Levine and Stoltzfus found a classification of $\mathcal{F}_{0} / \mathcal{F}_{0.5}$ modulo its torsion subgroup (Levine [24], Hillman [15], Cha [3], Stoltzfus [27]). They showed that

$$
\mathbb{Z}^{\infty} \cong \bigoplus_{p(t)} \mathbb{Z}^{r_{p}} \cong \mathcal{F}_{0} / \mathcal{F}_{0.5} / \text { torsion }
$$

where the sum is over all irreducible $p(t) \in \mathbb{Z}\left[t, t^{-1}\right]$ with $p(1)= \pm 1, p\left(t^{-1}\right) \doteq p(t)$, and where $r_{p}$ is the number of distinct pairs $(z, \bar{z})$ of unit-norm complex roots of $p(t)$. In this decomposition, a given knot has a nonzero $p(t)$-component only if $p(t)$ is a factor of its Alexander polynomial. It is important to note that the Alexander polynomial of a knot is not itself a concordance invariant, but there are concordance invariants given by signatures that are associated to its roots.

More recently, the work of Se-Goo Kim [18] and joint work of Se-Goo Kim and Taehee Kim [19] proved that

$$
\mathbb{Z}^{\infty} \cong \bigoplus_{p(t)} \mathbb{Z} \subset \mathcal{F}_{1} / \mathcal{F}_{1.5}
$$

Recent work of Cochran, Harvey and Leidy [10] aimed to generalize these results, and give evidence for an analogous but much more intricate decomposition for each 
$\mathcal{F}_{n} / \mathcal{F}_{n .5}$. For each sequence of polynomials $\mathcal{P}=\left(p_{1}(t), \ldots, p_{n}(t)\right)$, they were able to define a new filtration of $\mathcal{C}$, denoted $\left\{\mathcal{F}_{n}^{\mathcal{P}}\right\}$, such that $\mathcal{F}_{n} \subset \mathcal{F}_{n}^{\mathcal{P}}$ for all $n \in \frac{1}{2} \mathbb{Z}$. In this analysis, $p_{1}(t)$ could be thought of as a prime factor of the Alexander polynomial of the knot and the other $p_{i}(t)$ as being related to the higher-order Alexander polynomials.

All the examples they constructed have a $\mathcal{P}=\left(p_{1}(t), \ldots, p_{n}(t)\right)$ associated to them and these examples are nontrivial in $\mathcal{F}_{n} / \mathcal{F}_{n .5}^{\mathcal{P}}$, but if there is a knot associated to $\mathcal{Q}=\left(q_{1}(t), \ldots, q_{n}(t)\right)$, where $q_{i}(t)$ is strongly coprime to $p_{i}(t)$ (see Section 4.1) for some $i$, then it can be shown that this knot is trivial in $\mathcal{F}_{n} / \mathcal{F}_{n .5}^{\mathcal{P}}$. Therefore the quotient groups $\mathcal{F}_{n} / \mathcal{F}_{n .5}^{\mathcal{P}}$ act like sieves. This also justifies thinking of $\mathcal{F}_{n} / \mathcal{F}_{n .5}^{\mathcal{P}}$ as localizing $\mathcal{F}_{n} / \mathcal{F}_{n .5}$ at $\mathcal{P}$. The main result of [10] was that for $n \geq 1$,

$$
\bigoplus_{\mathbb{P}_{n}} \mathbb{Z}^{\infty} \subset \mathcal{F}_{n} / \mathcal{F}_{n .5}
$$

where $\mathbb{P}_{n}$ is an infinite set of pairwise strongly coprime $n$-tuples $\mathcal{P}=\left(p_{1}(t), \ldots, p_{n}(t)\right)$ of single-variable polynomials where $p_{i}(1)= \pm 1$. The knots constructed in [10] to prove these results will be referred to as CHL knots.

Most of the mentioned results, including all results of Cochran, Harvey and Leidy, involve the construction of knots via iterated satellite operations. The primary goal of the present work, stated in most generality, is to investigate if more can be uncovered about the $n$-solvable filtration by studying more general constructions of knots. The satellite operations used in $[9 ; 10]$ are referred to as infections by knots. In this paper we will be concerned with more general operations known as infections by string links.

Definition 1.1 Let $\left\{x_{1}, x_{2}, \ldots, x_{m}\right\}$ be $m$ fixed points in the interior of $D^{2}$. An $m$ component string link is a smooth proper embedding $L: \coprod_{i=1}^{m} I_{i} \rightarrow D^{2} \times I$ such that $L\left(0_{i}\right)=\left(x_{i}, 0\right)$ and $L\left(1_{i}\right)=\left(x_{i}, 1\right)$.

As with knots and links we often consider the string link to be the image of the embedding. Two string links are equivalent if there is an ambient isotopy from one to the other that fixes $\partial\left(D^{2} \times I\right)$. That is, if there is a smooth map $h:\left(D^{2} \times I\right) \times J \rightarrow\left(D^{2} \times I\right)$, $J \cong[0,1]$, such that for each $t \in J, h(x, t):\left(D^{2} \times I\right) \rightarrow\left(D^{2} \times I\right)$ is a diffeomorphism that fixes the boundary, $h(x, 0)=x$, for all $x \in\left(D^{2} \times I\right)$, and $h\left(I_{i}^{0}(s), 1\right)=I_{i}^{1}(s)$ for all $s \in I$. The trivial string link of $m$ components is given by $g: \coprod_{i=1}^{m} I_{i} \rightarrow D^{2} \times I$ such that $g\left(I_{i}(t)\right)=\left(x_{i}, t\right)$. Since cutting open a knot creates a string link of one component that does not depend on the choice of cutpoint, $\mathcal{K}$ is equivalent to the monoid of 1-component string links under the operation of stacking.

We define the complement of a string link to be $D^{2} \times I$ where a tubular neighborhood of each embedded arc is removed. In Figure 2 an example of such a string link is 
shown with its complement to the right. The meridian of a component of a string link is the simple closed curve, up to ambient isotopy, on the boundary of the tubular neighborhood of the component that has linking number +1 with that component. We call the set of such meridians the meridians of the string link. Note the set of meridians of a string link lie in the boundary of the complement of that string link. The longitude of a component of a string link is the simple closed curve, up to ambient isotopy, on the boundary of the complement of the string link that has +1 intersection number with the meridian of that component and has zero linking number with the core of the component. We call the set of such longitudes the longitudes of the string link. In Figure 2 the meridians, $\mu_{i}$, and longitudes, $\ell_{i}$, are shown on the boundary of the complement.
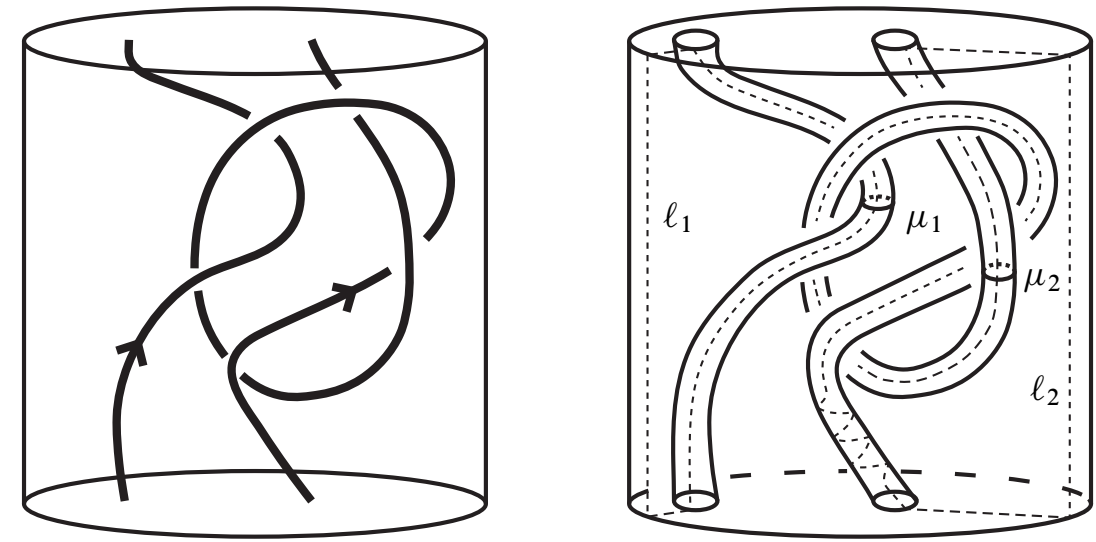

Figure 2: A string link and its complement

We now define infection by a string link. See Cochran, Friedl and Teichner [6, Section 2.2] for a thorough reference. By an $r$-multidisk $\mathbb{D}$, we mean the oriented disk $D^{2}$ together with $r$ ordered embedded open disks $D_{1}, \ldots, D_{r}$ (see Figure 3). Given a link $L \subset S^{3}$ we say that a map $\varphi: \mathbb{D} \rightarrow S^{3}$ of an $r$-multidisk into $S^{3}$ is proper if it is an embedding such that the image of the multidisk, denoted by $\mathcal{D}$, intersects the link components transversely and only in the images of the disks $D_{1}, \ldots, D_{r}$ as in Figure 3. We will refer to the image of the boundary curves of $\varphi\left(D_{1}\right), \ldots, \varphi\left(D_{r}\right)$ by $\eta_{1}, \ldots, \eta_{r}$.

If $\mathcal{R} \subset S^{3}$ is a knot, link or string link, $\mathcal{D} \subset S^{3}$ is the image of a properly embedded $r$-multidisk and $L$ is an $r$ component string link, then $\mathcal{R}_{\mathcal{D}}(L)$ is the knot, link or string link (respectively) obtained by tying the $r$ collections of strands of $\mathcal{R}$ that intersect the disks $\varphi\left(D_{1}\right), \ldots, \varphi\left(D_{r}\right)$ into the pattern of the string link $L$, where the strands 

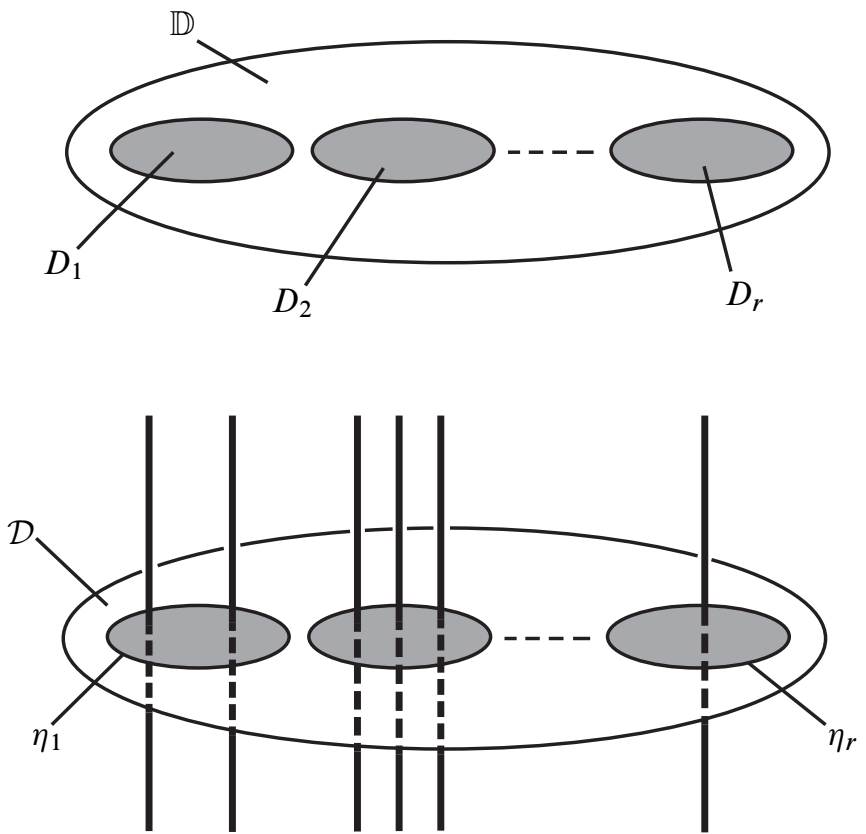

Figure 3: An $r$-multidisk and a properly embedded multidisk

linked by $\eta_{i}$ are identified with the $i^{\text {th }}$ component of $L$, such that the $i^{\text {th }}$ collection of strands are parallel copies of the $i^{\text {th }}$ component of $L$. In Figure 4, the result of this operation is shown for a particular $\mathcal{R}, \mathcal{D}$ and $L$.

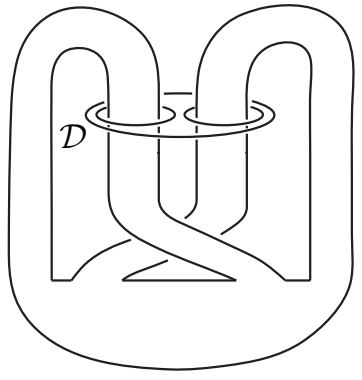

$\mathcal{R}$

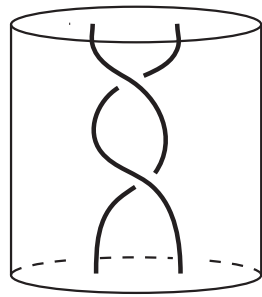

$L$

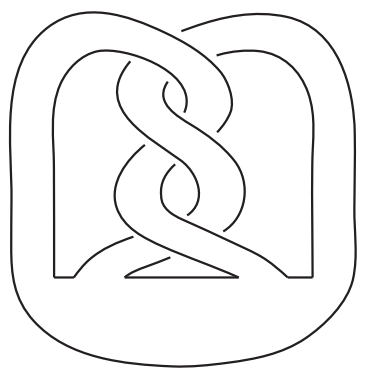

$\mathcal{R}_{\mathcal{D}}(L)$

Figure 4: Infection by a string link

It is important to note that while $L$ is infecting $\mathcal{R}$ at $\mathcal{D}$, the result is written as $\mathcal{R}_{\mathcal{D}}(L)$. We can and will view this as $\mathcal{R}_{\mathcal{D}}$ acting on $L$. 
Definition 1.2 Two $r$ component string links $L_{0}$ and $L_{1}$ are concordant if there exists a smooth embedding $H:\left(\bigsqcup_{r} I\right) \times I \rightarrow\left(D^{2} \times I\right) \times I$ that is transverse to the boundary such that $\left.H\right|_{\bigsqcup_{r} I \times 0}=L_{0},\left.H\right|_{\bigsqcup_{r} I \times 1}=L_{1}$ and for all $t \in I, H\left(0_{i}, t\right)=\left(\left(x_{i}, 0\right), t\right)$ and $H\left(1_{i}, t\right)=\left(\left(x_{i}, 1\right), t\right)$.

String link concordance defines an equivalence relation on the set of $r$ component string links. There is a binary operation defined on the set of string links called stacking. Given two string links $L_{0} \subset\left(D^{2} \times I\right)_{L_{0}}$ and $L_{1} \subset\left(D^{2} \times I\right)_{L_{1}}$, each with $r$ components, the result of stacking $L_{1}$ over $L_{0}$ is obtained by identifying $\left(D^{2} \times\{1\}\right)_{L_{0}}$ with $\left(D^{2} \times\{0\}\right)_{L_{1}}$. Note this operation is not in general commutative. The identity element is the trivial string link of $r$ components. The stacked product is well-defined on the string link concordance classes. String links concordant to the trivial string link of $r$ components are called slice string links with $r$ components. The inverse of the class of a string link $L,[L]$, is $[r \bar{L}]$, where $r \bar{L}$ is the mirror image of $L$ about $D^{2} \times\left\{\frac{1}{2}\right\}$ with reversed orientation. Therefore for each $r \geq 1$, the collection of concordance classes of string links with $r$ components with stacking form the $r$ component string link concordance group, $\mathcal{C}(r)$. Note $\mathcal{C}(1) \cong \mathcal{C}$ since any concordance may be assumed to be straight on an arc.

If $\mathcal{R}$ is a string link and $L$ is concordant to $L^{\prime}$, then $\mathcal{R}_{\mathcal{D}}(L)$ is concordant to $\mathcal{R}_{\mathcal{D}}\left(L^{\prime}\right)$. This is because $\mathcal{R}_{\mathcal{D}}(L)$ and $\mathcal{R}_{\mathcal{D}}\left(L^{\prime}\right)$ are obtained by cutting $\mathcal{R}$ along disks with boundary the $\eta_{i}$ of $\mathcal{D}$ and gluing in copies of $L$ and $L^{\prime}$ respectively such that the copies of the $i^{\text {th }}$ component are parallel. So one can obtain the needed concordance disks by cutting open the concordance disks created by crossing $\mathcal{R}$ with the unit interval, $I$, and gluing in copies of the concordance disks from $L$ to $L^{\prime}$. Since the copies of the $i^{\text {th }}$ components are parallel the concordance disks will not intersect. Therefore if $\mathcal{R}$ has $m$ components and $\mathcal{D}$ is properly embedded $r$-multidisk, then $\mathcal{R}_{\mathcal{D}}$ may be viewed as a operator, but not a homomorphism, from $\mathcal{C}(r)$ to $\mathcal{C}(m)$. In particular, if $\mathcal{R}$ is a knot then $\mathcal{R}_{\mathcal{D}}$ may be viewed as an operator from $\mathcal{C}(r)$ to $\mathcal{C}$.

There are a special class of these operators that will be of particular interest.

Definition 1.3 For any slice string link $\mathcal{R}$ of $m$ components and any properly embedded $r$-multidisk, $\mathcal{R}_{\mathcal{D}}: \mathcal{C}(r) \rightarrow \mathcal{C}(m)$ is a doubling operator if the linking number of each $\eta_{i}$ with each component of $\mathcal{R}$ is zero. (The linking number condition is equivalent to saying that the $\eta_{i}$ lie in $\pi_{1}\left(M_{\mathcal{R}}\right)^{(1)}$.)

We will call a doubling operator nontrivial as long as $\mathcal{R}$ has a nontrivial Alexander polynomial.

Doubling operators will be the cornerstone in building examples of knots in $\mathcal{F}_{n} / \mathcal{F}_{n .5}$. In Section 3.1 we will highlight their important features. In [10], doubling operators 
where $\mathcal{R}$ is a knot were considered. We shall refer to these as knot doubling operators. We consider iterations of these operations $\mathcal{R}_{\mathcal{D}_{n}}^{n} \circ \cdots \circ \mathcal{R}_{\mathcal{D}_{1}}^{1}$. The importance of these doubling operators is explained by the following propositions.

Proposition 1.4 [6, Proposition 1.7] Up to concordance, every knot in $\mathcal{F}_{0.5}$ is of the form $\mathcal{R}_{\mathcal{D}}(L)$, where $\mathcal{R}_{\mathcal{D}}$ is a doubling operator and $L$ is a string link with linking numbers zero.

This proposition demonstrates that knots obtained by doubling operations are general enough to study the entire structure of the $n$-solvable filtration.

Corollary 3.8 If $\mathcal{R}_{\mathcal{D}_{i}}^{i}, 1 \leq i \leq n$, are doubling operators, $\mathcal{R}^{n}$ is a knot and $K_{0} \in \mathcal{F}_{0}$, then

$$
\mathcal{R}_{\mathcal{D}_{n}}^{n} \circ \cdots \circ \mathcal{R}_{\mathcal{D}_{1}}^{1}\left(K_{0}\right) \in \mathcal{F}_{n}
$$

For each knot obtained by iterated doubling operators, we can obtain a sequence of polynomials $\mathcal{M}=\left(p_{1}\left(t_{1}, \ldots, t_{r}\right), \ldots, p_{n}\left(t_{1}, \ldots, t_{r}\right)\right)$, where $p_{i}$ is the Alexander polynomial of $\hat{\mathcal{R}}^{n-i+1}$, the closure of $\mathcal{R}^{n-i+1}$. (The multivariable Alexander polynomial of a link of $r$ components is a polynomial in $r$ variables.)

The construction of knots using knot doubling operators applied to knots in $\mathcal{F}_{0}$ accounts for nearly all previously mentioned results. In particular, the knots in the result (1) of Cochran, Harvey and Leidy are indexed over the set $\mathbb{P}_{n}$, which is a collection of sequences of single-variable polynomials that arise from iterated knot doubling operators.

In this paper we will generalize the notion of being strongly coprime in order to find multivariable polynomials that are strongly coprime to all single-variable polynomials. Furthermore, we find links that realize these polynomials as Alexander polynomials.

The main result of this paper is the following.

Theorem 6.4 For $n \geq 2$, there exists a knot, $K_{n}$, of infinite order in $\mathcal{F}_{n} / \mathcal{F}_{n .5}$ such that no multiple of $K_{n}$ is concordant to any knots created by two or more applications of nontrivial knot doubling operators to knots in $\mathcal{F}_{0}$. In particular,

$$
m K_{n} \notin \bigoplus_{\mathbb{P}_{n}} \mathbb{Z}^{\infty} \subset \mathcal{F}_{n} / \mathcal{F}_{n .5}
$$

for all $m \in \mathbb{Z}^{+}$. Thus, $m K_{n}$ is distinct from all CHL knots for all $n$ and $m \in \mathbb{Z}^{+}$. 


\subsection{Paper outline}

In Section 2 we discuss further infection by a string link. We then define the string link concordance group $\mathcal{C}(r)$ of string links of $r$ components.

In Section 3 we review the concept of commutator series developed in [10] and discuss how certain commutator series define filtrations of $\mathcal{C}(r)$. We finish the section by discussing the relationship between iterated infections and these filtrations. In particular we discuss their relationship with the $n$-solvable filtration, $\left\{\mathcal{F}_{n}\right\}$, resulting in the statement of Corollary 3.8.

In Section 4 we generalize the notion of a polynomial being strongly coprime to another polynomial to include multivariable polynomials. We prove the existence of links whose Alexander polynomials are strongly coprime to all single-variable polynomials. Additionally, by using Ore localizations, we extend the construction of Cochran, Harvey and Leidy to define the derived series localized at $\mathcal{M}, G_{\mathcal{M}}^{(n)}$, associated to a given sequence of multivariable polynomials $\mathcal{M}=\left(p_{1}\left(t_{1}, \ldots, t_{r}\right), \ldots, p_{n}\left(t_{1}, \ldots, t_{r}\right)\right)$. The derived series localized at $\mathcal{M}$ defines filtrations, $\left\{\mathcal{F}_{n}(r)^{\mathcal{M}}\right\}$, of $\mathcal{C}(r)$ such that $\mathcal{F}_{n}(r) \subset \mathcal{F}_{n}(r)^{\mathcal{M}}$ for all $n$.

In Section 5, we prove an important triviality result. We show that if

$$
\mathcal{Q}=\left(q_{n}\left(t_{1}, \ldots, t_{r}\right), \ldots, q_{1}\left(t_{1}, \ldots, t_{r}\right)\right)
$$

is a sequence of polynomials associated to a knot, $K$, obtained by doubling operators, and $\mathcal{M}=\left(p_{n}\left(t_{1}, \ldots, t_{r}\right), \ldots, p_{1}\left(t_{1}, \ldots, t_{r}\right)\right)$ is a sequence of polynomials such that $p_{i}$ is strongly coprime to $q_{i}$, for some $i$, then $K$ is trivial in $\mathcal{F}_{n} / \mathcal{F}_{n .5}^{\mathcal{M}}$.

Section 6 contains the most important result of the paper. It is a nontriviality result that proves that there is a knot obtained by string link doubling operators that has infinite order in $\mathcal{F}_{n} / \mathcal{F}_{n .5}^{\mathcal{M}}$, where $\mathcal{M}$ is a specific sequence of multivariable polynomials that includes a polynomial that is strongly coprime to all single variable polynomials. By applying the previously stated triviality result, this knot will not be concordant to any knots resulting from a composition of nontrivial knot doubling operators applied to a knot in $\mathcal{F}_{0}$. We conclude this section by affirming the statement of the main result, Theorem 6.4.

\section{String links}

\subsection{Infection by string links}

The closure, $\hat{L}$, of a string link, $L$, is obtained from $L \subset\left(D^{2} \times I\right)_{L}$ and a trivial string link $T \in\left(D^{2} \times I\right)_{T}$ with the same number of components by identifying 
$\left(D^{2} \times\{0\}\right)_{L}$ with $\left(D^{2} \times\{1\}\right)_{T},\left(D^{2} \times\{1\}\right)_{L}$ with $\left(D^{2} \times\{0\}\right)_{T}$ and $\left(\partial D^{2} \times\{t\}\right)_{L}$ with $\left(\partial D^{2} \times\{1-t\}\right)_{T}$ for all $0<t<1$. The result is a link in $S^{3}$. The closure of the string link from Figure 2 is shown in Figure 5.

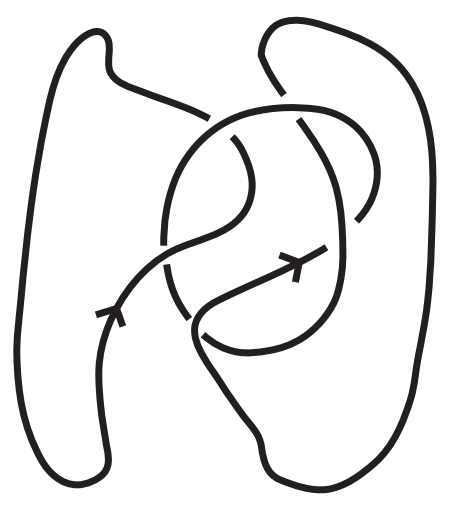

Figure 5: The closure of a string link

Suppose $L$ is a string link of $r$ components. Let $\mathcal{L}=\left(D^{2} \times I\right)-L$ denote its complement. Then the zero surgery of $\widehat{L}, M_{\hat{L}}$, can be obtained from $\mathcal{L}$ by gluing a genus $r$ handlebody, $\mathcal{H}$, to $\mathcal{L}$ so that the boundary of the meridional disks are identified with the longitudes $\ell_{i}, 1 \leq i \leq r$, of $L$.

Consider $\mathcal{R}_{\mathcal{D}}(L)$ for some string link $\mathcal{R}$, properly embedded multidisk $\mathcal{D}$ and string link $L$. We describe a cobordism $E$ whose boundary is the disjoint union of $M_{\widehat{\mathcal{R}}}$, $M_{\hat{L}}$ and $M_{\mathcal{R}_{\mathcal{D}}(L)}$, the zero-framed surgeries on $\widehat{\mathcal{R}}, \widehat{L}$ and $\widehat{\mathcal{R}_{\mathcal{D}}(L)}$ respectively. The cobordism $E$ is obtained from the union of $M_{\widehat{\mathcal{R}}} \times[0,1]$ and $M_{\widehat{L}} \times[0,1]$ by identifying $\left(\left(\mathcal{D}-\bigsqcup_{i} \varphi\left(E_{i}\right)\right) \times[0,1]\right) \times 0 \subset M_{\widehat{\mathcal{R}}} \times 0$ with $\mathcal{H} \times 0 \subset M_{\hat{L}} \times 0$ such that the meridians of $L$ are identified with the $\eta_{i}$ of $\mathcal{D}$. This cobordism has significant properties, which we will present in the following lemma.

Lemma 2.1 Let $K$ denote $\mathcal{R}_{\mathcal{D}}(L)$. With regard to $E$ above:

(1) The meridians of $L, \mu_{i} \in M_{\hat{L}}$, are isotopic in $E$ to both the $\eta_{i} \in M_{\hat{\mathcal{R}}}$, and to the longitudinal push-off of the $\eta_{i}, \ell_{\eta_{i}} \in M_{\widehat{K}}$.

(2) The inclusion map induces an epimorphism $\pi_{1}\left(M_{\widehat{K}}\right) \rightarrow \pi_{1}(E)$ whose kernel is the normal closure of the longitudes, $\ell_{i}$, of $L$ viewed in $M_{\hat{K}}$.

(3) The inclusion maps induce isomorphisms $H_{1}\left(M_{\widehat{K}}\right) \rightarrow H_{1}(E)$ and $H_{1}\left(M_{\widehat{\mathcal{R}}}\right) \rightarrow$ $H_{1}(E)$.

(4) The inclusion maps induce isomorphisms $H_{2}(E) \cong H_{2}\left(M_{\hat{K}}\right) \oplus H_{2}\left(M_{\hat{L}}\right) \cong$ $H_{2}\left(M_{\widehat{\mathcal{R}}}\right) \oplus H_{2}\left(M_{\widehat{L}}\right)$. 


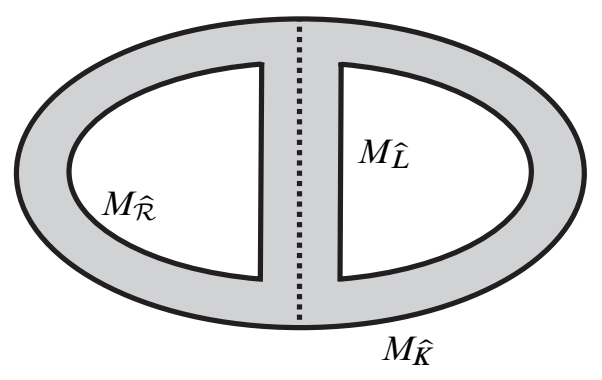

Figure 6: The cobordism $E$

Proof Property (1) is obvious from the definition of infection and of $E$.

Note that $E$ deformation retracts to $\bar{E}=M_{\widehat{K}} \cup \mathcal{H}$. Hence $\bar{E}$ can be obtained from $M_{\widehat{K}}$ by adding $r$ 2-cells along the longitudes of $L, \ell_{i}$, and capping off with a 3 -cell. Property (2) thus follows.

The first part of property (3) follows from the fact that $\ell_{i} \in \pi_{1}(\mathcal{L})^{(1)} \subset \pi_{1}\left(M_{\hat{K}}\right)^{(1)}$. By the description of $\bar{E}$ in the previous paragraph, since $\mathcal{H}=M_{\hat{L}}-\mathcal{L}$ we can observe that $\bar{E}=M_{\hat{K}} \cup_{\mathcal{L}} M_{\hat{L}}$. Therefore we have the Mayer-Vietoris sequence

$$
H_{2}(\mathcal{L}) \rightarrow H_{2}\left(M_{\widehat{K}}\right) \oplus H_{2}\left(M_{\widehat{L}}\right) \rightarrow H_{2}(\bar{E}) \rightarrow H_{1}(\mathcal{L}) \rightarrow H_{1}\left(M_{\widehat{K}}\right) \oplus H_{1}\left(M_{\widehat{L}}\right) .
$$

Since $H_{*}(\mathcal{L}) \cong H_{*}\left(\bigvee_{r} S^{1}\right), H_{2}(\mathcal{L})=0$. Thus, the first map of the sequence is the zero map. Also, since $H_{1}(\mathcal{L})$ is generated by the meridians of $L$ and these meridians survive in $H_{1}\left(M_{\hat{L}}\right)$, the last map is injective. Therefore, the third map is the zero map. Thus, the second map is an isomorphism, affirming the first part of property (4).

Consider the Mayer-Vietoris sequence

$$
\begin{aligned}
H_{2}(\mathcal{H}) \rightarrow H_{2}\left(M_{\hat{\mathcal{R}}} \times I\right) \oplus H_{2}\left(M_{\hat{L}}\right. & \times I) \rightarrow H_{2}(E) \rightarrow H_{1}(\mathcal{H}) \\
& \rightarrow H_{1}\left(M_{\widehat{\mathcal{R}}} \times I\right) \oplus H_{1}\left(M_{\hat{L}} \times I\right) \rightarrow H_{1}(E) \rightarrow 0 .
\end{aligned}
$$

Since $H_{2}(\mathcal{H})$ is zero, the first map is the zero map. As in the above paragraph, since $H_{1}(\mathcal{H})$ is generated by the meridians of $L$ that survive in $H_{1}\left(M_{\hat{L}} \times I\right)$, the fourth map is injective and thus the third map is the zero map. This affirms the second part of property (4).

Note that under the fourth map, the generators of $H_{1}(\mathcal{H})$ (the meridians of $L$ ) map onto the generators of $H_{1}\left(M_{\hat{L}} \times I\right)$. This implies that there is an isomorphism from $H_{1}\left(M_{\hat{\mathcal{R}}}\right)$ to $H_{1}(E)$. This affirms the second part of property (3). 
If $\mathcal{R}$ is a knot or link (rather than a string link) then there also exists a cobordism $E$ whose boundary is the disjoint union of $M_{\mathcal{R}}, M_{\hat{L}}$ and $M_{\mathcal{R}_{\mathcal{D}}(L)}$, with properties analogous to those in Lemma 2.1.

The following lemma will be important in the technical proof of Section 6 .

Lemma 2.2 The ordinary signature of $E, \sigma(E)$, vanishes.

Proof By Lemma 2.1(4) we know that $H_{2}(E ; \mathbb{Z}) \cong H_{2}\left(M_{\widehat{\mathcal{R}}} ; \mathbb{Z}\right) \oplus H_{2}\left(M_{\hat{L}} ; \mathbb{Z}\right)$. Since the second homology of $E$ with $\mathbb{Z}$ coefficients comes from its boundary, $\sigma(E)$ vanishes.

Lemma 2.3 If $L$ is a knot, then $\sigma_{\Gamma}^{2}(E)=0$ with respect to any coefficient system $\phi: \pi_{1}(E) \rightarrow \Gamma$.

The proof of this lemma is verbatim the proof of Lemma 2.4 in [9] except that we allow $\mathcal{R}$ to be a link of more than 1 -component.

\section{Commutator series and filtrations}

We begin with a review of work by Cochran, Harvey and Leidy [10] that introduced refinements of the $n$-solvable filtration derived from certain classes of group series that generalize the derived series.

Definition 3.1 [10, Definition 2.1] A commutator series is a function, $*$, that assigns to each group $G$ a nested sequence of normal subgroups

$$
\cdots \unlhd G_{*}^{(n+1)} \unlhd G_{*}^{(n)} \unlhd \cdots \unlhd G_{*}^{(0)} \equiv G
$$

such that $G_{*}^{(n)} / G_{*}^{(n+1)}$ is a torsion-free abelian group. (The torsion-free condition is used in order to avoid zero divisors in $\mathbb{Z}\left[G / G_{*}^{(n)}\right]$.)

The primary example that one should keep in mind is that of the rational derived series, $\left\{G_{r}^{(n)}\right\}$, where $G_{r}^{(0)} \equiv G$ and

$$
G_{r}^{(n+1)} \equiv\left\{x \in G_{r}^{(n)} \mid \exists k>0, x^{k} \in\left[G_{r}^{(n)}, G_{r}^{(n)}\right]\right\} .
$$

The rational derived series was used extensively in [9]. The following proposition is proved in [10]. 
Proposition 3.2 [10, Proposition 2.2] For any commutator series $\left\{G_{*}^{(n)}\right\}$

(1) $\left\{x \in G_{*}^{(n)} \mid \exists k>0, x^{k} \in\left[G_{*}^{(n)}, G_{*}^{(n)}\right]\right\} \subset G_{*}^{(n+1)}$ (and in particular $\left[G_{*}^{(n)}, G_{*}^{(n)}\right] \subset$ $G_{*}^{(n+1)}$, hence the name commutator series),

(2) $G_{r}^{(n)} \subset G_{*}^{(n)}$ (that is, every commutator series contains the rational derived series),

(3) $G / G_{*}^{(n)}$ is a poly-(torsion-free abelian) group (abbreviated PTFA),

(4) $\mathbb{Z}\left[G / G_{*}^{(n)}\right]$ and $\mathbb{Q}\left[G / G_{*}^{(n)}\right]$ are right (and left) Ore domains.

We will show that any commutator series that satisfies a weak form of functorality induces a filtration, $\mathcal{F}(m)_{n}^{*}$, on $\mathcal{C}(m)$.

Definition 3.3 For a given commutator series $*$, a string link of $m$ components, $L$, is an element of $\mathcal{F}(m)_{n}^{*}$ if the zero-framed surgery of the closure of $L, M_{\hat{L}}$, bounds a compact, connected, oriented, smooth 4-manifold $W$ such that:

(1) $H_{1}\left(M_{\hat{L}} ; \mathbb{Z}\right) \rightarrow H_{1}(W ; \mathbb{Z})$ is an isomorphism.

(2) $H_{2}(W ; \mathbb{Z})$ has a basis consisting of connected, compact, oriented surfaces, $\left\{J_{i}, D_{i} \mid 1 \leq i \leq l\right\}$, embedded in $W$ with trivial normal bundles, such that the surfaces are pairwise disjoint except that, for each $i, J_{i}$ intersects $D_{i}$ transversely once with positive sign.

(3) For each $i, \pi_{1}\left(J_{i}\right) \subset \pi_{1}(W)_{*}^{(n)}$ and $\pi_{1}\left(D_{i}\right) \subset \pi_{1}(W)_{*}^{(n)}$.

Additionally $L \in \mathcal{F}(m)_{n .5}^{*}$ if the following holds:

(4) For each $i, \pi_{1}\left(J_{i}\right) \subset \pi_{1}(W)_{*}^{(n+1)}$.

A 4-manifold, $W$, satisfying these conditions is called an $(n, *)$-solution (respectively an $(n .5, *)-$ solution) for $L$ and we say that $L$ is $(n, *)$-solvable (respectively $(n .5, *)-$ solvable) via $W$. When using this language there is a possibility for ambiguity as to the value of $m$. This will always be understood from context. In particular, when $m=1$ we drop the $(m)$ from the above notation. In the case where the commutator series is the derived series this is the $(n)$-solvable filtration defined by Cochran, Orr and Teichner in [11], denoted $\left\{\mathcal{F}_{n}\right\}$. Note that for any $n \in \frac{1}{2} \mathbb{Z}, \mathcal{F}_{n} \subset \mathcal{F}_{n}^{*}$.

A functorial commutator series is a commutator series such that for any group homomorphism $f: G \rightarrow \pi, f\left(G_{*}^{(n)}\right) \subset \pi_{*}^{(n)}$ for each $n$. For our purposes, we will need to discuss commutator series that satisfy a weaker form of functorality. 
Definition 3.4 [10, Definition 2.4] A commutator series $\left\{G_{*}^{(n)}\right\}$ is weakly functorial if for any homomorphism $f: G \rightarrow \pi$ that induces an isomorphism $G / G_{r}^{(1)} \cong \pi / \pi_{r}^{(1)}$ (ie, induces an isomorphism on $H_{1}(-; \mathbb{Q})$ ), we have $f\left(G_{*}^{(n)}\right) \subset \pi_{*}^{(n)}$ for each $n$.

Proposition 3.5 Suppose $*$ is a weakly functorial commutator series defined on the class of groups with $\beta_{1}=m$. Then $\left\{\mathcal{F}(m)_{n}^{*}\right\}_{n \geq 0}$ is a filtration of subgroups of $\mathcal{C}(m)$ :

$$
\cdots \subset \mathcal{F}(m)_{n+1}^{*} \subset \mathcal{F}(m)_{n .5}^{*} \subset \mathcal{F}(m)_{n}^{*} \subset \cdots \subset \mathcal{F}(m)_{0.5}^{*} \subset \mathcal{F}(m)_{0}^{*} \subset \mathcal{C}(m)
$$

Proof We sketch the proof. Let $L_{0}$ and $L_{1}$ be string links with $m$ components. If $L_{0}$ and $L_{1}$ are concordant, then their closures, $\hat{L}_{0}$ and $\hat{L}_{1}$, are concordant. It is well known that their zero-framed surgeries, $M_{\widehat{L}_{0}}$ and $M_{\widehat{L}_{1}}$, are homology cobordant via a 4 -manifold $C$ (obtained by performing zero framed surgery on the annuli). Suppose $L_{0} \in \mathcal{F}(m)_{n}^{*}$ via $W_{0}$. Let $W_{1}=W_{0} \cup C$ so that $\partial W_{1}=M_{\hat{L}_{1}}$. Note that the inclusion $W_{0} \hookrightarrow W_{1}$ induces isomorphisms on homology. By weak functorality, $\pi_{1}\left(W_{0}\right)_{*}^{(k)} \subset \pi_{1}\left(W_{1}\right)_{*}^{(k)}$ for every $k$. Then it is easy to see that $L_{1} \in \mathcal{F}(m)_{n}^{*}$ via $W_{1}$ using the surfaces from $W_{0}$. Therefore $\mathcal{F}(m)_{n}^{*}$ is closed under concordance.

We claim that $\mathcal{F}(m)_{n}^{*}$ is a subgroup. If $L$ is a slice string link of $m$ components then $M_{\widehat{L}}=\partial\left(B^{4}-\bigsqcup_{i} \Delta_{i}\right)$, where $\left(B^{4}-\bigsqcup_{i} \Delta_{i}\right)$ is the complement of slice disks, $\Delta_{i}$, of $\hat{L}$. Note that $H_{2}\left(B^{4}-\bigsqcup_{i} \Delta_{i}\right)=0$. Thus $L \in \mathcal{F}(m)_{n}^{*}$ via $\left(B^{4}-\bigsqcup_{i} \Delta_{i}\right)$ for any $n$ and any $*$. Since $-M_{\widehat{L}} \cong M_{-} \widehat{L}, \mathcal{F}(r)_{n}^{*}$ is closed under taking inverses in $\mathcal{C}$. Since $L J$ (the result of stacking $L$ on $J$ ) can be obtained by infecting $L$ with $J$ ( $L$ and $J$ both having $m$ components), there is a cobordism $E$ whose boundary consists of $M_{\widehat{L}}, M_{\widehat{J}}$, and $M_{\widehat{L J}}$ (see Lemma 2.1). If $L$ is $(n, *)$-solvable via $W_{L}$ and $J$ is $(n, *)$-solvable via $W_{J}$, then let $W=E \cup W_{L} \cup W_{J}$ so that $\partial W=M_{\widehat{L J}}$. The inclusions of $M_{\hat{L}} \hookrightarrow W$ and $M_{\widehat{J}} \hookrightarrow W$ induce isomorphisms on $H_{1}$. Also, the inclusion $M_{\widehat{L J}} \hookrightarrow W$ induces an isomorphism on $H_{1}$. (See Lemma 2.1.) Using weak functorality, one can then show that $L J$ is $(n, *)$-solvable via $W$ using the union of the surfaces from $W_{L}$ and $W_{J}$. (See the proof of Proposition 3.6 below.) Thus $\mathcal{F}(m)_{n}^{*}$ is closed under stacking of string links. Therefore $\mathcal{F}(m)_{n}^{*}$ is a filtration by subgroups of $\mathcal{C}(m)$.

\subsection{Doubling operators}

Proposition 3.6 For any weakly functorial commutator series $*$, if $\mathcal{R}_{\mathcal{D}}$ is a doubling operator where $\mathcal{R}$ is a string link of $m$ components, $\mathcal{D}$ is a properly embedded $r-$ multidisk with $\eta_{i} \in \pi_{1}\left(M_{\widehat{\mathcal{R}}}\right)_{*}^{(k)}$, for $1 \leq i \leq r$ and $L \in \mathcal{F}(r)_{n-k}$, then

$$
\mathcal{R}_{\mathcal{D}}(L) \in \mathcal{F}(m)_{n}^{*},
$$

where $\mathcal{R}_{\mathcal{D}}(L)$ is the result of infecting $\mathcal{R}$ along $\mathcal{D}$ with $L$. 
Proof Consider a slice string link $\mathcal{R}$, a properly embedded $r$-multidisk, $\mathcal{D}$, with $\eta_{i} \in \pi_{1}\left(M_{\widehat{\mathcal{R}}}\right)_{*}^{(k)}$, and a string link $L$ of $r$ components. Suppose $L \in \mathcal{F}(r)_{n-k}$ via $V$ and $S=B^{4}-\bigsqcup \Delta_{i}$, where $\Delta_{i}$ are slice disks for $\hat{\mathcal{R}}\left(\partial S=M_{\widehat{\mathcal{R}}}\right)$. Consider the cobordism $E$, described in Section 2.1, from $M_{\widehat{\mathcal{R}}} \sqcup M_{\hat{L}}$ to $M_{\widehat{K}}$, where $K=\mathcal{R}_{\mathcal{D}}(L)$. Call the result of gluing $E$ with $V$ and $S$ along common boundaries $W$ (Figure 7). Note the boundary of $W$ is $M_{\widehat{K}}$. We claim that $K \in \mathcal{F}(m)_{n}^{*}$ via $W$.

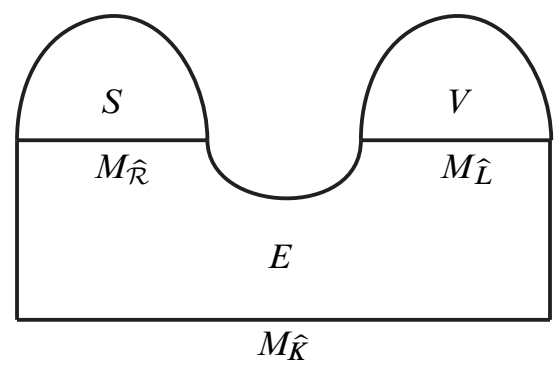

Figure 7: The result of gluing $E$ with $V$ and $S$ along common boundaries $W$

Consider the Mayer-Vietoris sequence

$$
H_{1}\left(M_{\hat{L}}\right) \rightarrow H_{1}(E) \oplus H_{1}(V) \rightarrow H_{1}\left(E \cup_{M_{\widehat{L}}} V\right) \rightarrow 0 .
$$

Since $V$ is an $n$-solution for $\widehat{L}, H_{1}(V)$ is isomorphic to $H_{1}\left(M_{\hat{L}}\right)$ and is generated by the meridians, $\mu_{i}$, of $\widehat{L}$. Therefore $H_{1}\left(E \cup_{M_{\widehat{L}}} V\right) \cong H_{1}(E)$.

Now consider the Mayer-Vietoris sequence

$$
H_{1}\left(M_{\widehat{\mathcal{R}}}\right) \rightarrow H_{1}\left(E \cup_{M_{\widehat{L}}} V\right) \oplus H_{1}(S) \rightarrow H_{1}(W) \rightarrow 0 .
$$

Since $H_{1}(S)$ is isomorphic to $H_{1}\left(M_{\widehat{\mathcal{R}}}\right)$ and is generated by the meridians of $\mathcal{R}$, $H_{1}(W) \cong H_{1}\left(E \cup_{M_{\widehat{L}}} V\right) \cong H_{1}(E)$. By property (3) of Lemma 2.1, $H_{1}(W) \cong$ $H_{1}\left(M_{\hat{K}}\right) \cong H_{1}\left(M_{\widehat{\mathcal{R}}}\right)$.

We will now show that the inclusion map $M_{\widehat{L}} \rightarrow V$ induces an isomorphism on $H_{1}$ and the zero map on $\mathrm{H}_{2}$. Consider the commutative diagram

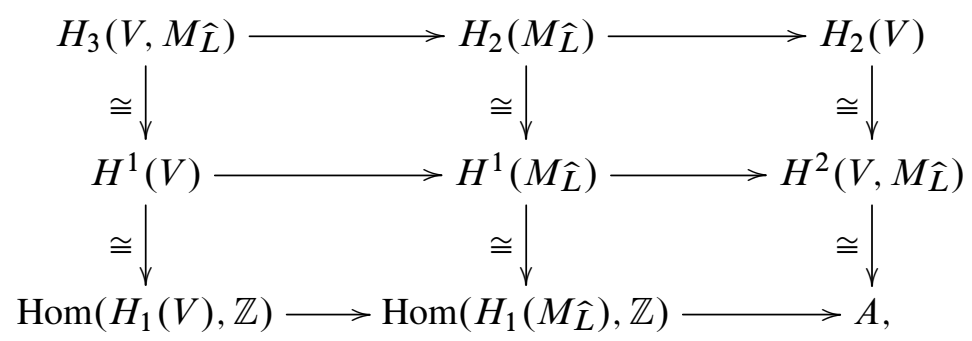

where $A$ is $\operatorname{Hom}\left(H_{2}\left(V, M_{\widehat{L}}\right), \mathbb{Z}\right) \oplus \operatorname{Ext}\left(H_{1}\left(V, M_{\widehat{L}}\right), \mathbb{Z}\right)$. 
The top row is a part of the long exact sequence of a pair. The set of isomorphisms from the top row to the middle row come from Poincare duality and the set of isomorphisms from the middle row to the bottom row come from the universal coefficient theorem and the fact that $\operatorname{Ext}\left(H_{0}(V), \mathbb{Z}\right)$ and $\operatorname{Ext}\left(H_{0}\left(M_{\hat{L}}\right), \mathbb{Z}\right)$ are both zero. Since there is an isomorphism from $H_{1}\left(M_{\hat{L}}\right) \rightarrow H_{1}(V)$, the left lower horizontal map is also an isomorphism. This implies that the right lower horizontal map is the zero map and thus all the right maps on each row are the zero map. In particular, the inclusion map from $M_{\hat{L}}$ to $V$ induces the zero map on $\mathrm{H}_{2}$.

Now consider the Mayer-Vietoris sequence

$$
H_{2}\left(M_{\hat{L}}\right) \stackrel{\phi}{\rightarrow} H_{2}(E) \oplus H_{2}(V) \rightarrow H_{2}\left(E \cup_{M_{\widehat{L}}} V\right) \rightarrow H_{1}\left(M_{\widehat{L}}\right) \rightarrow H_{1}(E) \oplus H_{1}(V) .
$$

Observe that the last map is injective since $H_{1}\left(M_{\hat{L}}\right) \rightarrow H_{1}(V)$ is an isomorphism. Thus, the second to last map is the zero map. Therefore, the second map is surjective. We have shown that $H_{2}\left(M_{\widehat{L}}\right) \rightarrow H_{2}(V)$ is the zero map and since $H_{2}(E) \cong H_{2}\left(M_{\widehat{\mathcal{R}}}\right) \oplus$ $H_{2}\left(M_{\hat{L}}\right)$ by property (4) of Lemma $2.1,\left(H_{2}(E) \oplus H_{2}(V)\right) / \operatorname{im}(\phi) \cong H_{2}\left(M_{\widehat{\mathcal{R}}}\right) \oplus$ $H_{2}(V)$. Therefore $H_{2}\left(E \cup_{M_{\widehat{L}}} V\right) \cong H_{2}\left(M_{\widehat{\mathcal{R}}}\right) \oplus H_{2}(V)$.

Consider the Mayer-Vietoris sequence

$$
\begin{aligned}
H_{2}\left(M_{\widehat{\mathcal{R}}}\right) \stackrel{\psi}{\rightarrow} H_{2}\left(E \cup M_{\widehat{L}} V\right) \oplus H_{2}(S) \rightarrow & H_{2}(W) \\
& \rightarrow H_{1}\left(M_{\widehat{\mathcal{R}}}\right) \rightarrow H_{1}\left(E \cup_{M_{\widehat{L}}} V\right) \oplus H_{1}(S) .
\end{aligned}
$$

Since the map $H_{1}\left(M_{\widehat{\mathcal{R}}}\right) \rightarrow H_{1}(S)$ is an isomorphism the last map is injective. Thus the third map is the zero map and therefore the second map is surjective. Recall that $H_{2}(S)=0$. Since $H_{2}\left(E \cup_{M_{\widehat{L}}} V\right) \cong H_{2}\left(M_{\widehat{\mathcal{R}}}\right) \oplus H_{2}(V)$ by the previous discussion, $H_{2}\left(E \cup_{M_{\hat{L}}} V\right) / \operatorname{im}(\psi) \cong H_{2}(V)$. Therefore $H_{2}(W) \cong H_{2}(V)$.

Since $V$ is an $(n-k)$-solution, $H_{2}(V)$ has a basis consisting of connected compact oriented surfaces $J_{s}, D_{s}$, such that $\pi_{1}\left(J_{S}\right) \subset \pi_{1}(V)^{(n-k)}$ and $\pi_{1}\left(D_{s}\right) \subset \pi_{1}(V)^{(n-k)}$. We claim that

$$
\pi_{1}(V) \subset \pi_{1}(W)_{*}^{(k)} .
$$

Assuming this, we would then have that

$$
\pi_{1}\left(J_{S}\right) \subset \pi_{1}(V)^{(n-k)} \subset\left(\pi_{1}(W)_{*}^{(k)}\right)^{(n-k)} \subset \pi_{1}(W)_{*}^{(n)} .
$$

(The last inclusion is from iterated applications of part (1) of Proposition 3.2.) The same argument works for $\pi_{1}\left(D_{S}\right)$.

To establish (2), consider the inclusion $j: \pi_{1}\left(M_{\hat{L}}\right) \rightarrow \pi_{1}(V)$. Recall that the meridians of $\hat{L}, \mu_{i}$, normally generate $\pi_{1}\left(M_{\hat{L}}\right)$. Thus by the following lemma proved by Cochran, Harvey and Leidy in [8] we know that the meridians of $\hat{L}$ normally generate $\pi_{1}(V) / \pi_{1}(V)^{(k)}$. 
Lemma 3.7 [8, Lemma 6.5] Suppose $\phi: A \rightarrow B$ is a group homomorphism that is surjective on abelianizations. Then, for any positive integer $k, \phi(A)$ normally generates $B / B^{(k)}$.

Since $\pi_{1}(V)^{(k)} \subset \pi_{1}(W)^{(k)} \subset \pi_{1}(W)_{*}^{(k)}$, we only need to show that $\mu_{i} \in \pi_{1}(W)_{*}^{(k)}$. We know by property (1) of Lemma 2.1 that the $\eta_{i}$ are isotopic in $E$ and hence in $W$ to $\eta_{i}$. By hypothesis, $\eta_{i} \in \pi_{1}\left(M_{\widehat{\mathcal{R}}}\right)_{*}^{(k)}$ and combined with the weak functorality of $*$ we can conclude that

$$
\eta_{i} \in \pi_{1}\left(M_{\widehat{\mathcal{R}}}\right)_{*}^{(k)} \subset \pi_{1}(W)_{*}^{(k)} .
$$

Thus $\pi_{1}\left(J_{S}\right) \subset \pi_{1}(W)_{*}^{(n)}$ and $\pi_{1}\left(D_{S}\right) \subset \pi_{1}(W)_{*}^{(n)}$.

We can consider particular iterations of doubling operators.

Corollary 3.8 If $\mathcal{R}_{\mathcal{D}_{i}}^{i}, 1 \leq i \leq n$, are doubling operators, $\mathcal{R}^{n}$ is a knot and $K \in \mathcal{F}_{0}$, then

$$
\mathcal{R}_{\mathcal{D}_{n}}^{n} \circ \cdots \circ \mathcal{R}_{\mathcal{D}_{1}}^{1}(K) \subset \mathcal{F}_{n} .
$$

Proof Apply Proposition 3.6 repeatedly.

We will have cause in this paper to consider infection of $\mathcal{R}$ by more than one string link. The above proof can be easily modified to obtain the following result.

Proposition 3.9 For any weakly functorial commutator series $*$, if $\mathcal{R}_{\mathcal{D}_{1} \sqcup \cdots \sqcup \mathcal{D}_{s}}$ is a doubling operator where $\mathcal{R}$ has $m$ components, $\eta_{i, 1}, \ldots, \eta_{i, m} \in \pi_{1}\left(M_{\widehat{\mathcal{R}}}\right)_{*}^{(k)}$ for all $i=1$ to $s$ and $L_{1}, \ldots, L_{s} \in \mathcal{F}(r)_{n-k}$, then

$$
\mathcal{R}_{\mathcal{D}_{1} \sqcup \cdots \sqcup \mathcal{D}_{s}}\left(L_{1}, \ldots, L_{s}\right) \in \mathcal{F}(m)_{n}^{*},
$$

where $\mathcal{R}_{\mathcal{D}_{1} \sqcup \cdots \sqcup \mathcal{D}_{s}}\left(L_{1}, \ldots, L_{s}\right)$ is the result of infecting $\mathcal{R}$ along $\mathcal{D}_{i}$ with $L_{i}$ for all $i$

\section{Strongly coprime polynomials and localization at $\mathcal{M}$}

In [10], Cochran, Harvey and Leidy defined the derived series localized at $\mathcal{P}$ where $\mathcal{P}=\left(p_{1}(t), \ldots, p_{n}(t), \ldots\right)$ is any sequence of nonzero elements of $\mathbb{Q}\left[t, t^{-1}\right]$. In this section we will generalize the results of these authors in order to define the derived series localized at $\mathcal{M}$, where $\mathcal{M}$ is a sequence of nonzero multivariable Laurent polynomials. Suppose that $p\left(t_{1}, \ldots, t_{m}\right)$ is a nonzero element of the Laurent polynomial ring $\mathbb{Q}\left[t_{1}^{ \pm 1}, \ldots, t_{m}^{ \pm 1}\right]$ and $a_{1}, \ldots, a_{m} \in A$, where $A$ is a torsion-free abelian group. Then the group homomorphism $\mathbb{Z}^{m} \rightarrow A$ given by $t_{i} \mapsto a_{i}$ induces a ring homomorphism $\mathbb{Q}\left[t_{1}^{ \pm 1}, \ldots, t_{m}^{ \pm 1}\right] \rightarrow \mathbb{Q} A$. Thus $p\left(a_{1}, \ldots, a_{m}\right) \in \mathbb{Q} A$ can be viewed as the image of $p$ under this map. Note that if $p(1,1, \ldots, 1) \neq 0$ then $p\left(a_{1}, \ldots, a_{m}\right)$ is nonzero. 


\subsection{Strongly coprime}

Definition 4.1 Suppose $p, q \in \mathbb{Q}\left[t_{1}^{ \pm 1}, \ldots, t_{m}^{ \pm 1}\right]$. We say $p$ is strongly coprime to $q$, denoted $(\widetilde{p, q})=1$, if for any finitely generated free abelian group, $F$, and any $a_{i}$ and $b_{i}$ in $F, 1 \leq i \leq m$, such that the set $\left\{a_{1}, \ldots, a_{m}\right\}$ is $\mathbb{Z}$-linearly independent in $F$, $p\left(a_{1}, \ldots, a_{m}\right)$ is relatively prime to $q\left(b_{1}, \ldots, b_{m}\right)$ in $\mathbb{Q} F$. (Note that $\mathbb{Q} F$ is a unique factorization domain.)

Note that this definition is asymmetric since $\left\{b_{1}, \ldots, b_{m}\right\}$ need not be linearly independent.

In the case where $p$ and $q$ are single-variable polynomials, the linear independence condition is superfluous and therefore reduces to the definition of strongly coprime polynomials used in [10].

Definition 4.2 [10, Definition 4.4] Suppose $p(t), q(t) \in \mathbb{Q}\left[t, t^{-1}\right]$ are said to be strongly coprime, denoted $(\widetilde{p, q})=1$, if for any finitely generated free abelian group $F$ and any nontrivial $a, b \in F, p(a)$ is relatively prime to $q(b)$ in $\mathbb{Q} F$.

Strongly coprime is a strictly stronger condition than coprime. For example consider $p(t)=t-2$ and $q(t)=t-4$. These polynomials are coprime in $\mathbb{Q}\left[t, t^{-1}\right]$, but they are not strongly coprime since $p(a)$ and $q\left(a^{2}\right)$ have a common factor of $(a-2)$ in $\mathbb{Q}\left[a, a^{-1}\right]$.

Example 4.3 Consider the ribbon knot in Figure 8 where the $-k$ inside the box symbolizes $k \geq 1$ full negative twists between the bands.

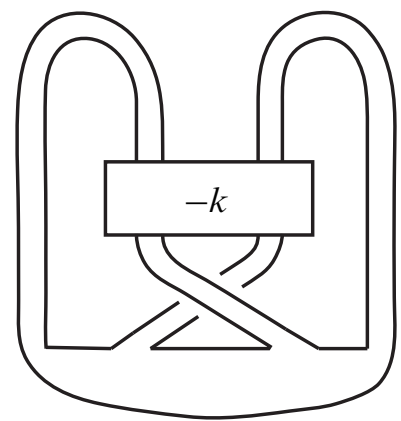

Figure 8: The ribbon knot $\mathfrak{R}^{k}$

The Alexander polynomial of $\mathfrak{R}^{k}$ is

$$
\mathfrak{p}_{k} \doteq(k t-(k+1))((k+1) t-k) .
$$


It was shown in [10, Example 4.10] that if $k \neq \ell$ then $\mathfrak{p}_{k}$ is strongly coprime to $\mathfrak{p}_{\ell}$. This example was instrumentally important toward the results of [10]. We will now define an important family of ribbon links that will be crucial to establishing our new result.

Example 4.4 Consider the ribbon link in Figure 9 where the $-k$ inside the box symbolizes $k \geq 1$ full negative twists between the bands.

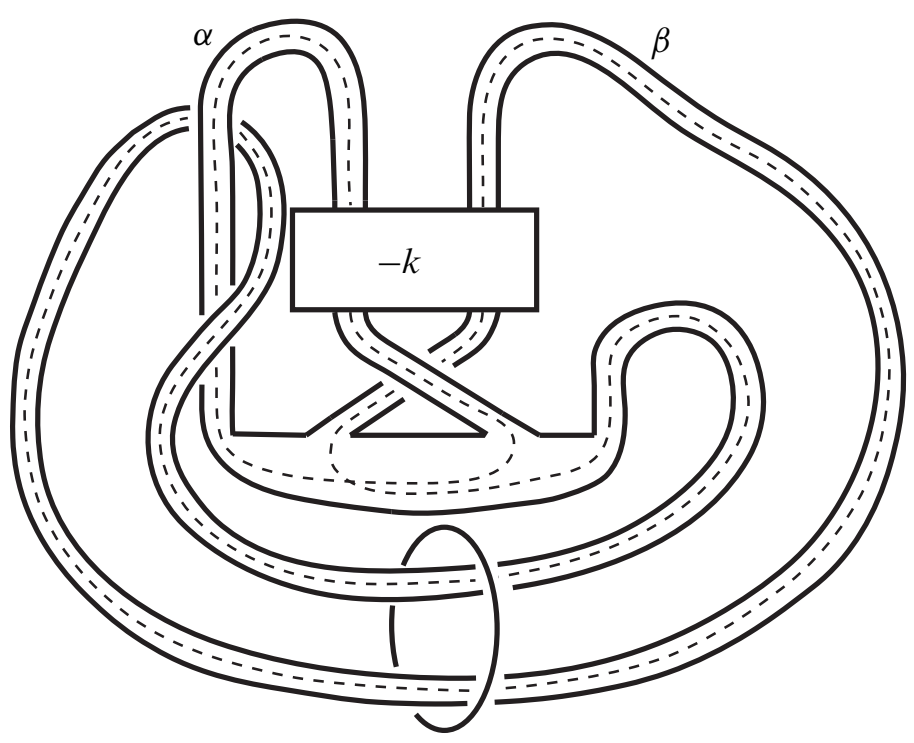

Figure 9: The ribbon link $\hat{\mathfrak{L}}^{k}$

There is a covering space of the complement of $\widehat{\mathfrak{L}}^{k}$ associated with the infinite cyclic subgroup of $\pi_{1}\left(\hat{\mathfrak{L}}^{k}\right)$ generated by the meridian of the unknotted component. This covering space contains infinitely many lifts of the knotted component. The following Seifert matrix, $A$, can be obtained by calculating the $\ell k\left(e_{i}, e_{j}^{+}\right)$matrix, where $\alpha$ and $\beta$ are a choosing set of generates (see Figure 9) for a particular lift of the Seifert surface of the knotted component. Let $t_{1}$ represent a generator for the group of deck transformations of said covering space. Then:

$$
\begin{array}{ccc} 
& \alpha & \beta \\
\alpha & 0 & k-t_{1}^{-1} \\
\beta & (k+1)-t_{1} & 0
\end{array}
$$

A presentation matrix for the homology of the universal abelian cover of the complement of $\hat{\mathfrak{L}}^{k}$ is obtained by calculating $A-t_{2} A^{*}$, where $A^{*}$ is the transpose of $A$ with the 
natural involution on $\mathbb{Q}\left[t_{1}, t_{1}^{-1}\right]$. (This need for the involution comes from the fact that the previously stated Seifert form of the homology of the infinite lifts of the Seifert surface, viewed as a $\mathbb{Q}\left[t_{1}, t_{1}^{-1}\right]$ module, is a Hermitian form.) The presentation matrix can be calculated as follows:

$$
\left(\begin{array}{cc}
0 & k-t_{1}^{-1}-\left(k_{1}-1\right) t_{2}+t_{1}^{-1} t_{2} \\
(k+1)-t_{1}-k t_{2}+t_{1} t_{2} & 0
\end{array}\right)
$$

Up to multiplication by units of $\mathbb{Q}\left[t_{1}^{ \pm 1}, t_{2}^{ \pm 1}\right]$, the Alexander polynomial of $\widehat{\mathfrak{L}}^{k}$ is

$$
\mathfrak{m}_{k} \doteq\left((k+1)-t_{1}-k t_{2}+t_{1} t_{2}\right)\left((k+1)-t_{1}^{-1}-k t_{2}^{-1}+t_{1}^{-1} t_{2}^{-1}\right) .
$$

This calculation can be verified by using the techniques of Example 7 of [26, page 194].

Lemma 4.5 For each $k \geq 1, \mathfrak{m}_{k}$ is strongly coprime to all (nonconstant) singlevariable polynomials.

We point out the importance of the linear independence condition in the definition of strongly coprime. If $p\left(t_{1}, \ldots, t_{m}\right) \in \mathbb{Q}\left[t_{1}{ }^{ \pm 1}, \ldots, t_{m}{ }^{ \pm 1}\right], q\left(t_{1}\right)=p\left(t_{1}, \ldots, t_{1}\right) \in$ $\mathbb{Q}\left[t_{1}, t_{1}^{-1}\right]$ and $a$ is nonzero in $F$, some finitely generated free abelian group, then $p(a, \ldots, a)=q(a) \in \mathbb{Q} F$. This situation is avoided by including the linear independence condition.

Proof The following fact will be useful throughout the proof.

Fact 4.6 Suppose $a_{1}, \ldots, a_{m}$ are elements of a finitely generated free abelian group $F$ that are $\mathbb{Z}$-linearly independent. Then there is a monomorphism $\phi: F \rightarrow F^{\prime}$, where $F^{\prime}$ is another finitely generated free abelian group with a basis $\left\{x_{1}, \ldots, x_{m}, \ldots, x_{n}\right\}$, such that $\phi\left(a_{i}\right)=x_{i}^{k_{1}}$ for $i=1, \ldots, m$.

Let $q(t)$ be an arbitrary single-variable polynomial and $F$ an arbitrary finitely generated free abelian group. Let $a, b$ and $c$ be elements of $F$ such that $\{a, b\}$ is a $\mathbb{Z}$-linearly independent set in $F$.

We want to show that $\mathfrak{m}_{k}(a, b)$ and $q(c)$ have no common factor in $\mathbb{Q} F$. We will proceed with a proof by contradiction. Suppose that $\mathfrak{m}_{k}(a, b)$ and $q(c)$ have a common factor. Then $q(c)$ has a common factor with either $\mathfrak{m}_{k}^{1}(a, b)$ or $\mathfrak{m}_{k}^{2}(a, b)$, where $\mathfrak{m}_{k}^{1}\left(t_{1}, t_{2}\right)=(k+1)-t_{1}-k t_{2}+t_{1} t_{2}$ and $\mathfrak{m}_{k}^{2}\left(t_{1}, t_{2}\right)=(k+1)-t_{1}^{-1}-k t_{2}^{-1}+t_{1}^{-1} t_{2}^{-1}$. Note if $c$ is trivial, $q(c)$ is a unit and thus $q(c)$ will have no common factors with $\mathfrak{m}_{k}(a, b)$. Therefore suppose that $c$ is nontrivial in $F$.

Suppose that $q(c)$ has a common factor with $\mathfrak{m}_{k}^{1}(a, b)$ in $F$. 
Case $I$ Suppose that $\{a, c\}$ is a $\mathbb{Z}$-linearly independent set in $\boldsymbol{F}$ By the fact above, there exists a monomorphism $\phi: F \rightarrow F^{\prime}, F^{\prime}$ a finitely generated free abelian group with a basis including $x, y$ and $z$ such that $\phi(c)=x^{n}, \phi(a)=y^{m}$ and $\phi(b)=x^{\ell_{1}} y^{\ell_{2}} z^{\ell_{3}}$, where $n$ and $m$ are nonzero integers and each $\ell_{i}$ is an integer. Then $\phi(q(c))=q\left(x^{n}\right)$ and

$$
\phi\left(\mathfrak{m}_{k}^{1}(a, b)\right)=\mathfrak{m}_{k}^{1}\left(y^{m}, x^{\ell_{1}} y^{\ell_{2}} z^{\ell_{3}}\right)=(k+1)-y^{m}-k x^{\ell_{1}} y^{\ell_{2}} z^{\ell_{3}}+y^{m} x^{\ell_{1}} y^{\ell_{2}} z^{\ell_{3}} .
$$

Since, by assumption, $q(c)$ has a common factor with $\mathfrak{m}_{k}^{1}(a, b)$ in $\mathbb{Q} F$, it follows that $\phi(q(c))$ has a common factor with $\phi\left(\mathfrak{m}_{k}^{1}(a, b)\right)$ in $\mathbb{Q} F^{\prime}$. If $\{a, b, c\}$ were $\mathbb{Z}$-linearly independent, $\phi$ can be chosen so that $\phi(b)=z^{\ell_{3}}$. But then $\phi(q(c))$ would have no common factors with $\phi\left(\mathfrak{m}_{k}^{1}(a, b)\right)$ in $F^{\prime}$. Therefore $b \in \operatorname{span}\{a, c\}$ so $\ell_{3}=0$. Thus $\phi(q(c))=q\left(x^{n}\right)$ and $\phi\left(\mathfrak{m}_{k}^{1}(a, b)\right)=\mathfrak{m}_{k}^{1}\left(y^{m}, x^{\ell_{1}} y^{\ell_{2}}\right)=(k+1)-y^{m}-k x^{\ell_{1}} y^{\ell_{2}}+$ $y^{m} x^{\ell_{1}} y^{\ell_{2}}$.

Since $\phi(q(c))$ and $\phi\left(\mathfrak{m}_{k}^{1}(a, b)\right)$ have a common factor in $\mathbb{Q}\left[x^{ \pm 1}, y^{ \pm 1}\right]$, they will have a common factor in $\mathbb{C}\left[x^{ \pm 1}, y^{ \pm 1}\right]$. Note $\phi(q(c))=q\left(x^{n}\right) \in \mathbb{C}\left[x^{ \pm 1}\right]$ factors over $\mathbb{C}\left[x^{ \pm 1}\right]$ into linear factors of the form $(x-\alpha)$ for some nonzero $\alpha \in \mathbb{C}$. Thus $\phi\left(\mathfrak{m}_{k}(b, c)\right)$ must have a factor in $\mathbb{C}\left[x^{ \pm 1}, y^{ \pm 1}\right]$ of the form $(x-\alpha)$.

Therefore

$$
0=\mathfrak{m}_{k}^{1}\left(y^{m}, \alpha^{\ell_{1}} y^{\ell_{2}}\right)=(k+1)-y^{m}-k \alpha^{\ell_{1}} y^{\ell_{2}}+\alpha^{\ell_{1}} y^{m+\ell_{2}} .
$$

Case Ia Suppose $\ell_{2}=\mathbf{0}$ Then

$0=\mathfrak{m}_{k}^{1}\left(y^{m}, \alpha^{\ell_{1}} y^{\ell_{2}}\right)=(k+1)-y^{m}-k \alpha^{\ell_{1}}+\alpha^{\ell_{1}} y^{m}=\left(k+1-k \alpha^{\ell_{1}}\right)+\left(\alpha^{\ell_{1}}-1\right) y^{m}$.

Therefore $\alpha^{\ell_{1}}=1$ and the constant term of $\mathfrak{m}_{k}^{1}\left(y^{m}, \alpha^{\ell_{1}} y^{\ell_{2}}\right)$ is $k+1-k=1$. This is a contradiction.

Case Ib Suppose $\ell_{2} \neq \mathbf{0}$ and $\boldsymbol{m}+\boldsymbol{\ell}_{\mathbf{2}} \neq \mathbf{0}$ Then $\alpha^{\ell_{1}}=0$ and the constant term of $\mathfrak{m}_{k}^{1}\left(y^{m}, \alpha^{\ell_{1}} y^{\ell_{2}}\right)$ is $(k+1)$. This is a contradiction.

Case Ic Suppose $\boldsymbol{m}+\ell_{2}=\mathbf{0}$ Thus

$$
0=\mathfrak{m}_{k}^{1}\left(y^{m}, \alpha^{\ell_{1}} y^{\ell_{2}}\right)=(k+1)-y^{m}-k \alpha^{\ell_{1}} y^{-m}+\alpha^{\ell_{1}} .
$$

Since $-m \neq 0, k \alpha^{\ell_{1}}=0$. Thus $\alpha^{\ell_{1}}=0$ and $\mathfrak{m}_{k}^{1}\left(y^{m}, \alpha^{\ell_{1}} y^{\ell_{2}}\right)$ has a constant term of $(k+1)$. This is a contradiction.

Case II Suppose that $\{a, c\}$ is a $\mathbb{Z}$-linearly dependent set in $\boldsymbol{F}$ Then $\{b, c\}$ is a $\mathbb{Z}$-linearly independent set in $F$.

By Fact 4.6 above there exists a monomorphism $\phi^{\prime}: F \rightarrow F^{\prime \prime}, F^{\prime \prime}$ a finitely generated free abelian group with a basis including $x$, and $y$ such that $\phi^{\prime}(c)=x^{n}$ 
and $\phi^{\prime}(b)=y^{m}$, where $n$ and $m$ are nonzero integers. Since $a$ and $c$ are $\mathbb{Z}-$ linearly dependent, $\phi^{\prime}(a)=x^{\ell}$ for some integer $\ell$. Then $\phi^{\prime}(q(c))=q\left(x^{n}\right)$ and $\phi^{\prime}\left(\mathfrak{m}_{k}^{1}(a, b)\right)=\mathfrak{m}_{k}^{1}\left(x^{\ell}, y^{m}\right)=(k+1)-x^{\ell}-k y^{m}+x^{\ell} y^{m}$.

Since, by assumption, $q(c)$ has a common factor with $\mathfrak{m}_{k}^{1}(a, b)$ in $\mathbb{Q} F$, it follows that $\phi^{\prime}(q(c))$ has a common factor with $\phi^{\prime}\left(\mathfrak{m}_{k}^{1}(a, b)\right)$ in $\mathbb{Q} F^{\prime \prime}$.

As in Case I, $\phi^{\prime}(q(c))$ and $\phi^{\prime}\left(\mathfrak{m}_{k}^{1}(a, b)\right)$ must have a common factor in $\mathbb{C}\left[x^{ \pm 1}, y^{ \pm 1}\right]$. Since $\phi^{\prime}(q(a))=q\left(x^{n}\right)$ factors over $\mathbb{C}\left[x^{ \pm 1}\right]$ into linear factors of the form $(x-\alpha)$ for some nonzero $\alpha \in \mathbb{C}, \phi^{\prime}\left(\mathfrak{m}_{k}^{1}(a, b)\right)$ must have a factor in $\mathbb{C}\left[x^{ \pm 1}, y^{ \pm 1}\right]$ of the form $(x-\alpha)$.

Therefore, $0=\mathfrak{m}_{k}^{1}\left(\alpha^{\ell}, y^{m}\right)=(k+1)-\alpha^{\ell}-k y^{m}+\alpha^{\ell} y^{m}$. Thus,

$$
(k+1)-\alpha^{\ell}=0 \quad \text { and } \quad \alpha^{\ell}-k=0 .
$$

This is a contradiction. Therefore $q(c)$ does not have a common factor with $\mathfrak{m}_{k}^{1}(a, b)$ in $F$.

Suppose that $q(c)$ has a common factor with $\mathfrak{m}_{k}^{2}(a, b)$ in $F$. We have that for some monomorphism $\psi: F \rightarrow F^{\prime}, \psi(q(c))$ has a common factor with $\psi\left(\mathfrak{m}_{k}^{2}(a, b)\right)$ of the form $x-\beta$ in $\mathbb{C}\left[x^{ \pm 1}, y^{ \pm 1}\right]$ for some nonzero $\beta \in \mathbb{C}$.

Note that $\mathfrak{m}_{k}^{2}(a, b)=\mathfrak{m}_{k}^{1}\left(a^{-1}, b^{-1}\right)$. Thus $\psi(q(c))$ must have a common factor with $\psi\left(\mathfrak{m}_{k}^{1}(a, b)\right)$ of the form $x-(1 / \beta)$ in $\mathbb{C}\left[x^{ \pm 1}, y^{ \pm 1}\right]$. It has already been shown that this is a contradiction.

Therefore $q(c)$ has no common factor with either $\mathfrak{m}_{k}^{1}(a, b)$ or $\mathfrak{m}_{k}^{2}(a, b)$.

We will now define a right divisor set that will be used to define the derived series localized at $\mathcal{M}$.

Definition 4.7 Suppose $A \triangleleft \Gamma$ where $A$ is a torsion-free abelian group and $\mathbb{Q} \Gamma$ is a right Ore domain. If $p \in \mathbb{Q}\left[t_{1}^{ \pm 1}, \ldots, t_{m}{ }^{ \pm 1}\right]$ is nonzero, then set

$S_{p}^{\Gamma, A}=\left\{q_{1}\left(a_{1_{1}}, \ldots, a_{m_{1}}\right) \ldots q_{r}\left(a_{1_{r}}, \ldots, a_{m_{r}}\right) \mid \widetilde{\left(p, q_{i}\right)}=1, q_{i}(1, \ldots, 1) \neq 0, a_{i_{j}} \in A\right\}$.

Note that $S_{p}^{\Gamma, A} \subset \mathbb{Q} A \subset \mathbb{Q} \Gamma$. When $\Gamma$ and $A$ are clear, we will suppress them from the notation.

Note that $S_{p}^{\Gamma, A}$ is closed under the natural involution on $\mathbb{Q} \Gamma$, where

$$
\sum_{i=0}^{n} c_{i} g_{i} \mapsto \sum_{i=0}^{n} c_{i} g_{i}^{-1}
$$


Proposition 4.8 We have $S_{p}^{\Gamma, A}$ is a right divisor set of $\mathbb{Q} \Gamma$. In addition, $S_{p}^{\Gamma, A}$ is functorial in the sense that for any homomorphism $\psi:(\Gamma, A) \rightarrow\left(\Gamma^{\prime}, A^{\prime}\right)$, we have $\psi\left(S_{p}^{\Gamma, A}\right) \subset S_{p}^{\Gamma^{\prime}, A^{\prime}}$. If additionally $\psi: A \rightarrow A^{\prime}$ is surjective, then $\psi\left(S_{p}^{\Gamma, A}\right)=S_{p}^{\Gamma^{\prime}, A^{\prime}}$.

Proof We have defined $S_{p}^{\Gamma, A}$ so that it is a multiplicatively closed subset of $\mathbb{Q} A$ that contains 1 (the empty product). Certainly $q_{i}\left(a_{1_{i}}, \ldots, a_{m_{i}}\right) \neq 0$ since $q_{i}(1, \ldots, 1) \neq 0$. Since $A$ is torsion-free abelian, $\mathbb{Q} A$ is a commutative domain. Therefore $0 \in S_{p}^{\Gamma, A}$. Thus $S_{p}^{\Gamma, A}$ is a right divisor set of $\mathbb{Q} A$. For any $g \in \Gamma$ and $a_{1}, \ldots, a_{m} \in A$,

$$
g^{-1} q\left(a_{1}, \ldots, a_{m}\right) g=q\left(g^{-1} a_{1} g, \ldots, g^{-1} a_{m} g\right)=q\left(a_{1}^{\prime}, \ldots, a_{m}^{\prime}\right),
$$

for some $a_{1}^{\prime}, \ldots, a_{m}^{\prime} \in A$, since $A \triangleleft \Gamma$. Thus $S_{p}^{\Gamma, A}$ is $\Gamma$-invariant and by [10, Proposition 4.1], $S_{p}^{\Gamma, A}$ is a right divisor set of $\mathbb{Q} \Gamma$.

Given a homomorphism $\psi:(\Gamma, A) \rightarrow\left(\Gamma^{\prime}, A^{\prime}\right)$ with $A \triangleleft \Gamma$ and $A^{\prime} \triangleleft \Gamma^{\prime}, \psi$ induces a ring homomorphism $\psi: \mathbb{Q} A \rightarrow \mathbb{Q} A^{\prime}$ with respect to which $\psi\left(q\left(a_{1}, \ldots, a_{m}\right)\right)=$ $q\left(\psi\left(a_{1}\right), \ldots, \psi\left(a_{m}\right)\right)$. Therefore $\psi\left(S_{p}^{\Gamma, A}\right) \subset S_{p}^{\Gamma^{\prime}, A^{\prime}}$. If additionally $\psi: A \rightarrow A^{\prime}$ is a surjection then for a given $q\left(a_{1}^{\prime}, \ldots, a_{m}^{\prime}\right)$, where $a_{j}^{\prime} \in A^{\prime}$, there are $a_{j} \in A$ such that $\psi\left(a_{j}\right)=a_{j}^{\prime}$. Therefore $\psi\left(q\left(a_{1}, \ldots, a_{m}\right)\right)=q\left(\psi\left(a_{1}\right), \ldots, \psi\left(a_{m}\right)\right)=q\left(a_{1}^{\prime}, \ldots, a_{m}^{\prime}\right)$. Hence

$$
S_{p}^{\Gamma^{\prime}, A^{\prime}}=\psi\left(S_{p}^{\Gamma, A}\right) .
$$

We say that $\mathbb{Q} \Gamma S_{p}^{-1}$ is $\mathbb{Q} \Gamma$ localized at $p$. If $M$ is a right $\mathbb{Q} \Gamma$-module then we say that $M S_{p}^{-1}=M \otimes_{\mathbb{Q} \Gamma} \mathbb{Q} \Gamma S_{p}^{-1}$ is $M$ localized at $p$.

Over a commutative domain, localizing a module $M$ at a prime ideal $\langle p\rangle$ kills all torsion in $M$ except $\langle p\rangle$-torsion. A statement similar to this is also true in our context. The following theorem clarifies this point and is vital to the proof of our main theorem. This proof also establishes the purpose behind the definition of strongly coprime.

Theorem 4.9 Suppose $A \triangleleft \Gamma$ where $A$ is a torsion-free abelian group and $\mathbb{Q} \Gamma$ is a right Ore domain. Let $p^{\prime}$ be a factor of a nonzero polynomial $p \in \mathbb{Q}\left[t_{1}{ }^{ \pm 1}, \ldots, t_{m}^{ \pm 1}\right]$. Then for any linearly independent sets $\left\{a_{1_{j}}, \ldots, a_{m_{j}}\right\} \in A$,

$$
\frac{\mathbb{Q} \Gamma}{p^{\prime}\left(a_{1_{1}}, \ldots, a_{m_{1}}\right) \ldots p^{\prime}\left(a_{1_{k}}, \ldots, a_{m_{k}}\right) \mathbb{Q} \Gamma} \text { is } S_{p} \text {-torsion-free, }
$$

that is,

$$
\begin{aligned}
& \frac{\mathbb{Q} \Gamma}{p^{\prime}\left(a_{1_{1}}, \ldots, a_{m_{1}}\right) \ldots p^{\prime}\left(a_{1_{k}}, \ldots, a_{m_{k}}\right) \mathbb{Q} \Gamma} \\
& \rightarrow \frac{\mathbb{Q} \Gamma}{p^{\prime}\left(a_{1_{1}}, \ldots, a_{m_{1}}\right) \ldots p^{\prime}\left(a_{1_{k}}, \ldots, a_{m_{k}}\right) \mathbb{Q} \Gamma} S_{p}^{-1}
\end{aligned}
$$


is a monomorphism; whereas for any $q \in \mathbb{Q}\left[t_{1}^{ \pm 1}, \ldots, t_{m}^{ \pm 1}\right]$, where $(\widetilde{p, q})=1$ and $q(1, \ldots, 1) \neq 0$,

$$
\frac{\mathbb{Q} \Gamma}{q\left(a_{1}, \ldots, a_{m}\right) \mathbb{Q} \Gamma} \text { is } S_{p} \text {-torsion, that is, } \frac{\mathbb{Q} \Gamma}{q\left(a_{1}, \ldots, a_{m}\right) \mathbb{Q} \Gamma} S_{p}^{-1}=0 \text {. }
$$

Proof We will start by addressing the first claim. Suppose that $x \in \mathbb{Q} \Gamma$ represents an element

$$
[x] \in \frac{\mathbb{Q} \Gamma}{p^{\prime}\left(a_{1_{1}}, \ldots, a_{m_{1}}\right) \cdots p^{\prime}\left(a_{1_{k}}, \ldots, a_{m_{k}}\right) \mathbb{Q} \Gamma}
$$

that is $S_{p}$-torsion. We will show that $[x]=0$ proving that

$$
\mathbb{Q} \Gamma / p^{\prime}\left(a_{1_{1}}, \ldots, a_{m_{1}}\right) \cdots p^{\prime}\left(a_{1_{k}}, \ldots, a_{m_{k}}\right) \mathbb{Q} \Gamma
$$

is $S_{p}$-torsion-free. We know that

$$
x s=p^{\prime}\left(a_{1_{1}}, \ldots, a_{m_{1}}\right) \cdots p^{\prime}\left(a_{1_{k}}, \ldots, a_{m_{k}}\right) y
$$

for some $s \in S_{p}$ and $y \in \mathbb{Q} \Gamma$.

Since $A \subset \Gamma, \mathbb{Q} \Gamma$, viewed as a left $\mathbb{Q} A$-module, is free on the right cosets of $A$ in $\Gamma$ (Passman [25, Chapter 1, Lemma 1.3]). Thus, after fixing a set of coset representatives, any $x \in \mathbb{Q} \Gamma$ has a unique decomposition

$$
x=\sum_{\gamma} x_{\gamma} \gamma
$$

where the sum is over the coset representatives $\{\gamma \in \Gamma\}$ and $x_{\gamma} \in \mathbb{Q} A$. Thus, we obtain

$$
\left(\sum_{\gamma} x_{\gamma} \gamma\right) s=p^{\prime}\left(a_{1_{1}}, \ldots, a_{m_{1}}\right) \cdots p^{\prime}\left(a_{1_{k}}, \ldots, a_{m_{k}}\right) \sum_{\gamma} y_{\gamma} \gamma
$$

and hence

$$
\sum_{\gamma}\left(x_{\gamma} s^{\gamma^{-1}}\right) \gamma=\sum_{\gamma}\left(p^{\prime}\left(a_{1_{1}}, \ldots, a_{m_{1}}\right) \cdots p^{\prime}\left(a_{1_{k}}, \ldots, a_{m_{k}}\right) y_{\gamma}\right) \gamma
$$

where $s^{\gamma^{-1}}=\gamma s \gamma^{-1} \in S_{p}$. (Recall that $S_{p}$ is closed under the action of $\Gamma$.) Thus, for each coset representative $\gamma$ we obtain the equation

$$
x_{\gamma} s^{\gamma^{-1}}=p^{\prime}\left(a_{1_{1}}, \ldots, a_{m_{1}}\right) \cdots p^{\prime}\left(a_{1_{k}}, \ldots, a_{m_{k}}\right) y_{\gamma},
$$

which can be viewed as an equation in $\mathbb{Q} A$. Since $s^{\gamma^{-1}} \in S_{p}$, for each $\gamma$, we have $s^{\gamma^{-1}}=q_{1}\left(b_{1_{1}}, \ldots, b_{m_{1}}\right) \cdots q_{r}\left(b_{1_{r}}, \ldots, b_{m_{r}}\right)$ for $b_{i_{j}} \in A$ and $q_{i} \in \mathbb{Q}\left[t_{1}{ }^{ \pm 1}, \ldots, t_{m}^{ \pm 1}\right]$ (all depending on $\gamma$ ), where $\widetilde{\left(p, q_{i}\right)}=1$ and $q_{i}(1, \ldots, 1) \neq 0$. Thus we have $x_{\gamma} q_{1}\left(b_{1_{1}}, \ldots, b_{m_{1}}\right) \cdots q_{r}\left(b_{1_{r}}, \ldots, b_{m_{r}}\right)=p^{\prime}\left(a_{1_{1}}, \ldots, a_{m_{1}}\right) \cdots p^{\prime}\left(a_{1_{k}}, \ldots, a_{m_{k}}\right) y_{\gamma}$. 
This may be viewed as an equation in $\mathbb{Q} F_{\gamma}$ for some free abelian group $F_{\gamma} \subset A$ of finite rank. Since $\widetilde{\left(p, q_{i}\right)}=1$ and $p^{\prime}$ is a factor of $p$, the greatest common divisor, in $\mathbb{Q} F_{\gamma}$, of $p^{\prime}\left(a_{1_{j}}, \ldots, a_{m_{j}}\right)$ and $q_{i}\left(b_{1_{i}}, \ldots, b_{m_{i}}\right)$ is a unit. Thus, for each $\gamma$ and each $j$, $p^{\prime}\left(a_{1_{j}}, \ldots, a_{m_{j}}\right)$ divides $x_{\gamma}$ in $\mathbb{Q} F_{\gamma}$. Thus $p^{\prime}\left(a_{1_{j}}, \ldots, a_{m_{j}}\right)$ divides each $x_{\gamma}$ in $\mathbb{Q} A$, so

$$
x=p^{\prime}\left(a_{1_{1}}, \ldots, a_{m_{1}}\right) \cdots p^{\prime}\left(a_{1_{k}}, \ldots, a_{m_{k}}\right) \sum_{\gamma} x_{\gamma}^{\prime} \gamma,
$$

which is in $p^{\prime}\left(a_{1_{1}}, \ldots, a_{m_{1}}\right) \cdots p^{\prime}\left(a_{1_{k}}, \ldots, a_{m_{k}}\right) \mathbb{Q} \Gamma$. This implies that $[x]=0$. This finishes the proof of the first claim.

We will now address the second claim of the theorem. By hypothesis, we have $q\left(a_{1}, \ldots, a_{m}\right) \in S_{p}$. Recall that the kernel of the map

$$
M \equiv \frac{\mathbb{Q} \Gamma}{q\left(a_{1}, \ldots, a_{m}\right) \mathbb{Q} \Gamma} \rightarrow \frac{\mathbb{Q} \Gamma}{q\left(a_{1}, \ldots, a_{m}\right) \mathbb{Q} \Gamma} S_{p}^{-1} \equiv M S_{p}^{-1}
$$

is the $S_{p}$-torsion submodule of $M$. Note that $M$ is a cyclic right $\mathbb{Q} \Gamma$-module generated by $[1] \in M$, where $1 \in \mathbb{Q} \Gamma$. Certainly $[1] q\left(a_{1}, \ldots, a_{m}\right)=\left[q\left(a_{1}, \ldots, a_{m}\right)\right]=$ 0 in $M$. Thus the generator of $M$ is $S_{p}$-torsion and therefore $M$ is $S_{p}$-torsion.

\subsection{The derived series localized at $\mathcal{M}$}

We will now define a specific family of commutator series that will be used extensively throughout the rest of the paper. The constructions rely on the techniques of [10, Section 3] and Definition 4.7.

Let $\mathcal{M}=\left(p_{1}\left(t_{1}, \ldots, t_{m}\right), \ldots, p_{n}\left(t_{1}, \ldots, t_{m}\right), \ldots\right)$ be a sequence of nonzero elements of $\mathbb{Q}\left[t_{1}^{ \pm 1}, \ldots, t_{m}^{ \pm 1}\right]$. For each such $\mathcal{M}$ we will recursively define a functorial partial commutator series that we call the derived series localized at $\mathcal{M}$.

Given any group $G$, set $G_{\mathcal{M}}^{(0)} \equiv G$ and suppose inductively $G_{\mathcal{M}}^{(n)}$ has been defined so that $G_{\mathcal{M}}^{(k)} / G_{\mathcal{M}}^{(k+1)}$ is torsion-free abelian for each $k<n$. Then $G / G_{\mathcal{M}}^{(n)}$ is a PTFA group, so $\mathbb{Q}\left[G / G_{\mathcal{M}}^{(n)}\right]$ is a right Ore domain. Now consider

$$
\Gamma=G / G_{\mathcal{M}}^{(n)} \quad \text { and } \quad A=G_{\mathcal{M}}^{(n-1)} / G_{\mathcal{M}}^{(n)} \triangleleft \Gamma .
$$

Note that $A$ is torsion-free abelian. Thus, by Proposition 4.8, we have that $S_{p_{n}}$ is a right divisor set of $\mathbb{Q}\left[G / G_{\mathcal{M}}^{(n)}\right]$. We now define $G_{\mathcal{M}}^{(n+1)}$.

Definition 4.10 The derived series localized at $\mathcal{M}$ is given by $G_{\mathcal{M}}^{(0)} \equiv G$ and, for $n>0$,

$$
G_{\mathcal{M}}^{(n+1)} \equiv \operatorname{ker}\left(G_{\mathcal{M}}^{(n)} \rightarrow \frac{G_{\mathcal{M}}^{(n)}}{\left[G_{\mathcal{M}}^{(n)}, G_{\mathcal{M}}^{(n)}\right]} \rightarrow \frac{G_{\mathcal{M}}^{(n)}}{\left[G_{\mathcal{M}}^{(n)}, G_{\mathcal{M}}^{(n)}\right]} \otimes_{\mathbb{Z}\left[G / G_{\mathcal{M}}^{(n)}\right]} \mathbb{Q}\left[G / G_{\mathcal{M}}^{(n)}\right] S_{p_{n}}^{-1}\right) .
$$

If $n=0$ we understand that $S_{p_{n}}=\{1\}$. 
Note that $G_{\mathcal{M}}^{(n)} /\left[G_{\mathcal{M}}^{(n)}, G_{\mathcal{M}}^{(n)}\right]$ is not only an abelian group but also a right $\mathbb{Z}\left[G / G_{\mathcal{M}}^{(n)}\right]-$ module where the action is induced by conjugation $\left(x * g=g^{-1} x g\right.$ for any $g \in G$ and $\left.x \in G_{\mathcal{M}}^{(n)}\right)$. We are also using the fact that $\mathbb{Q}\left[G / G_{\mathcal{M}}^{(n)}\right] S_{p_{n}}^{-1}$ is a left $\mathbb{Z}\left[G / G_{\mathcal{M}}^{(n)}\right]$-module. It is easily observed that $\left[G_{\mathcal{M}}^{(n)}, G_{\mathcal{M}}^{(n)}\right] \subset G_{\mathcal{M}}^{(n+1)}$. The second map in this composition should really be viewed as first tensoring with $\mathbb{Q}$, which kills the $\mathbb{Z}$-torsion, and then inverting $S_{p_{n}}$. It follows that $G_{\mathcal{M}}^{(n)} / G_{\mathcal{M}}^{(n+1)}$ is a torsion-free abelian group (since $\mathbb{Q}\left[G / G_{\mathcal{M}}^{(n)}\right] S_{p_{n}}^{-1}$ is a rational vector space). Hence this procedure recursively defines a commutator series (or rather a partial commutator series up to $G_{\mathcal{M}}^{(n+1)}$ ) which depends on $\mathcal{M}$. (As is true for all commutator series, $G_{\mathcal{M}}^{(1)}=G_{r}^{(1)}$, the first term of the rational derived series.)

Theorem 4.11 If $\mathcal{M}=\left(p_{1}\left(t_{1}, \ldots, t_{m}\right), \ldots, p_{n}\left(t_{1}, \ldots, t_{m}\right), \ldots\right)$, then $\left\{G_{\mathcal{M}}^{(i)}\right\}$ is a functorial commutator series.

Proof Suppose $G$ and $B$ are groups and $\psi: G \rightarrow B$ is a homomorphism. We will show, by induction on $n$, that $\psi\left(G_{\mathcal{M}}^{(n)}\right) \subset B_{\mathcal{M}}^{(n)}$. This holds for $n=0$. Suppose this holds for $n$. We want to show $\psi\left(G_{\mathcal{M}}^{(n+1)}\right) \subset B_{\mathcal{M}}^{(n+1)}$. By [10, Proposition 3.2], it suffices to verify that, for each $0 \leq i \leq n, \psi\left(S_{p_{i}}^{G}\right) \subset S_{p_{i}}^{B}$. Since $i \leq n$, the induction hypothesis guarantees that $\psi$ induces a homomorphism of pairs

$$
\psi:\left(G / G_{\mathcal{M}}^{(i)}, G_{\mathcal{M}}^{(i-1)} / G_{\mathcal{M}}^{(i)}\right) \rightarrow\left(B / B_{\mathcal{M}}^{(i)}, B_{\mathcal{M}}^{(i-1)} / B_{\mathcal{M}}^{(i)}\right) .
$$

From the second part of Proposition 4.8, $\psi\left(S_{p_{i}}^{G}\right) \subset S_{p_{i}}^{B}$ for $0 \leq i \leq n$.

The following basic result is analogous to Proposition 4.17 in [10].

Proposition 4.12 If $\phi: A \rightarrow B$ is surjective and $\operatorname{ker} \phi \subset A_{\mathcal{M}}^{(m)}$, then $\phi$ induces isomorphisms $A / A_{\mathcal{M}}^{(n)} \cong B / B_{\mathcal{M}}^{(n)}$ for all $n \leq m$. In particular, $\left(A / A_{\mathcal{M}}^{(n)}\right)_{\mathcal{M}}^{(n)}=0$.

\section{Distinguishing concordance classes: Triviality}

Let $\mathcal{M}=\left(p_{1}\left(t_{1}, \ldots t_{m}\right), \ldots, p_{n}\left(t_{1}, \ldots, t_{m}\right), \ldots\right)$ be a sequence of nonzero elements of $\mathbb{Q}\left[t_{1}^{ \pm 1}, \ldots t_{m}^{ \pm 1}\right]$. By Theorem 4.11 we know that the derived series localized at $\mathcal{M}$, denoted $\left\{G_{\mathcal{M}}^{(n)}\right\}$, is a functorial commutator series. Thus by Proposition 3.5 there is a corresponding filtration, $\left\{\mathcal{F}_{n}^{\mathcal{M}}\right\}$, of the knot concordance group.

\section{Definition 5.1 Given}

$\mathcal{M}=\left(p_{1}\left(t_{1}, \ldots t_{m}\right), \ldots, p_{n}\left(t_{1}, \ldots, t_{m}\right)\right)$ and $\mathcal{Q}=\left(q_{1}\left(t_{1}, \ldots, t_{r}\right), \ldots, q_{n}\left(t_{1}, \ldots, t_{r}\right)\right)$,

we say that $\mathcal{M}$ is strongly coprime to $\mathcal{Q}$ if, for some $k \geq 1, \widetilde{\left(p_{k}, q_{k}\right)}=1$; otherwise we say that $\mathcal{M}$ is isogenous to $\mathcal{Q}$. 
We will concern ourselves with knots of the form $K=\mathcal{R}_{\mathcal{D}_{n}}^{n} \circ \cdots \circ \mathcal{R}_{\mathcal{D}_{1}}^{1}\left(K_{0}\right)$, where $K_{0} \in \mathcal{F}_{0}, \mathcal{R}_{\mathcal{D}_{i}}^{i}$ are doubling operators and $\mathcal{R}^{n}$ is a knot. By Corollary 3.8 we know that $K \in \mathcal{F}_{n}$ and thus $K \in \mathcal{F}_{n}^{\mathcal{M}}$ for any $\mathcal{M}$. Let $\mathcal{Q}=\left(q_{n}\left(t_{1}, \ldots, t_{m}\right), \ldots, q_{1}\left(t_{1}, \ldots, t_{m}\right)\right)$, where $q_{i}$ is the product of the $\eta_{i}^{j}$ of $\mathcal{D}_{i}$ in $\mathcal{A}\left(\mathcal{R}^{i}\right)$. The following result shows that for certain doubling operators, if $\mathcal{M}$ is strongly coprime to $\mathcal{Q}$ then $K \in \mathcal{F}_{n+1}^{\mathcal{M}}$ and therefore $K$ is trivial in $\mathcal{F}_{n} / \mathcal{F}_{n .5}^{\mathcal{M}}$, where $\mathcal{F}_{n} / \mathcal{F}_{n .5}^{\mathcal{M}}$ is defined as the quotient of $\mathcal{F}_{n} /\left(\mathcal{F}_{n} \cap \mathcal{F}_{n .5}^{\mathcal{M}}\right)$. This justifies thinking of $\mathcal{F}_{n} / \mathcal{F}_{n .5}^{\mathcal{M}}$ as localizing $\mathcal{F}_{n} / \mathcal{F}_{n .5}$ at $\mathcal{M}$.

Theorem 5.2 Suppose that $K=\mathcal{R}_{\mathcal{D}_{n}}^{n} \circ \cdots \circ \mathcal{R}_{\mathcal{D}_{1}}^{1}\left(K_{0}\right)$ with $K_{0} \in \mathcal{F}_{0}, \mathcal{R}^{n}$ is a knot and the $\eta_{i}^{j}$ of $\mathcal{D}_{i}$ lie in the torsion submodule of $\mathcal{A}\left(\mathcal{R}^{i}\right)$ (this is always satisfied if $\mathcal{R}^{i}$ is a knot). Let $\mathcal{Q}=\left(q_{n}\left(t_{1}, \ldots, t_{r}\right), \ldots, q_{1}\left(t_{1}, \ldots, t_{r}\right)\right)$ be the order of the $\eta_{i}^{j}$ of $\left(\mathcal{D}_{n}, \ldots, \mathcal{D}_{1}\right)$ in $\left(\mathcal{A}\left(\mathcal{R}^{n}\right), \ldots, \mathcal{A}\left(\mathcal{R}^{1}\right)\right)$ (note the descending index). If $\mathcal{M}=\left(p_{1}\left(t_{1}, \ldots, t_{r}\right), \ldots, p_{n}\left(t_{1}, \ldots, t_{r}\right)\right)$ is strongly coprime to $\mathcal{Q}$ then $K \in \mathcal{F}_{n+1}^{\mathcal{M}}$. Thus $K$ is trivial in $\mathcal{F}_{n} / \mathcal{F}_{n .5}^{\mathcal{M}}$.

The following proof is analogous to [10, Theorem 6.2] except the use of string link doubling operators and the derived series localized at $\mathcal{M}$ will be considered.

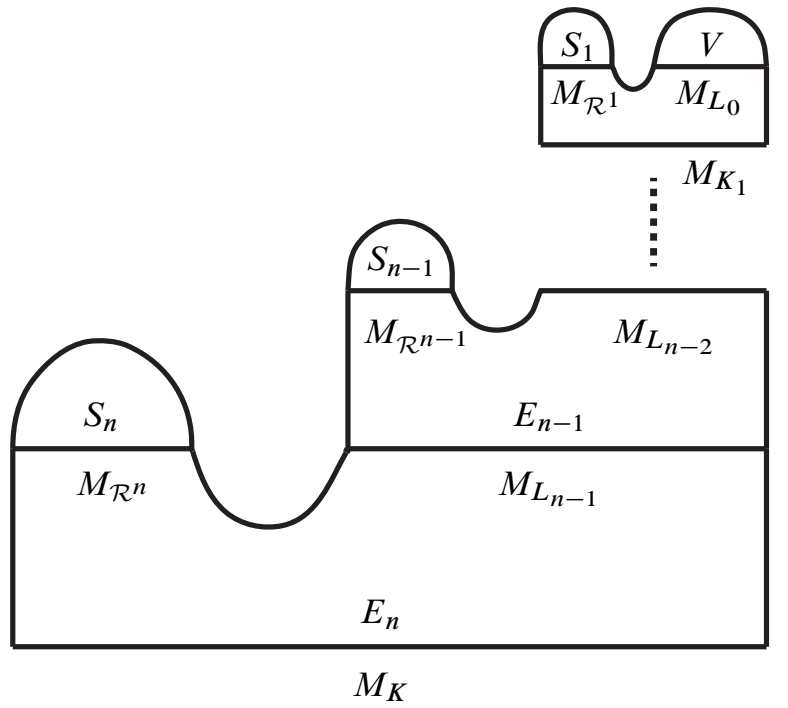

Figure 10: The 4-manifold $Z$

Proof We will recursively define $L_{i}=\mathcal{R}_{\mathcal{D}_{i}}^{i}\left(L_{i-1}\right)$ for $i=1, \ldots, n$. Note that $K=L_{n}$. Since $L_{i}=\mathcal{R}_{\mathcal{D}_{i}}^{i}\left(L_{i-1}\right)$ there is a cobordism $E_{i}$ (see Section 2.1) whose boundary is $M_{L_{i}} \sqcup M_{L_{i-1}} \sqcup M_{\mathcal{R}^{i}}$. Consider $X=E_{n} \cup E_{n-1} \cup \cdots \cup E_{1}$, obtained by gluing $E_{i}$ to $E_{i-1}$ along their common boundary component $M_{K_{i-1}}$. The boundary 
of $X$ is the disjoint union of $M_{K_{0}}, M_{\mathcal{R}^{n}}, \ldots, M_{\mathcal{R}^{1}}$ and $M_{K}$. For $1 \leq i \leq n$, let $S_{i}$ denote the exterior of slice disks in $B^{4}$ for the slice link $\mathcal{R}^{i}$. Since $K_{0} \in \mathcal{F}_{0}$ there is a zero solution $V$ for $K_{0}$. In fact, it is known that we can choose $V$ such that $\pi_{1}(V) \cong \mathbb{Z}$ so that the meridian $\mu_{0}$ of $K_{0}$ generates $\pi_{1}(V)$ [12, Section 5]. Gluing $V$ and all the $S_{i}$ to $X$, we obtain a 4 -manifold, $Z$ as shown schematically in Figure 10. Note that $\partial Z=M_{K}$.

We claim that

$$
K \in \mathcal{F}_{n} \text { via } Z
$$

and, if $\mathcal{M}$ is strongly coprime to $\mathcal{Q}$, then

$$
K \in \mathcal{F}_{n+1}^{\mathcal{M}} \text { via } Z \text {. }
$$

First, as in the proof of Proposition 3.6, a series of Mayer-Vietoris arguments implies that $H_{2}(Z) \cong H_{2}(V)$. Since $K_{0} \in \mathcal{F}_{0}$ via $V, H_{2}(V)$ has a basis of connected compact oriented surfaces, $\left\{J_{s}, D_{s}\right\}$, embedded in $V$ with trivial normal bundles such that the surfaces are pairwise disjoint except that, for each $s, J_{s}$ intersects $D_{s}$ transversely in a single point with positive sign (see Definition 3.3). We claim that

$$
\mu_{0} \in \pi_{1}(Z)^{(n)},
$$

where $\mu_{0}$ is the meridian of $K_{0}$, and if $\mathcal{M}$ is strongly coprime to $\mathcal{Q}$ then

$$
\mu_{0} \in \pi_{1}(Z)_{\mathcal{M}}^{(n+1)} \text {. }
$$

Then, since $\mu_{0}$ generates $\pi_{1}(V)$,

$$
\pi_{1}\left(J_{S}\right) \subset \pi_{1}(V) \subset \pi_{1}(Z)^{(n)},
$$

and if $\mathcal{M}$ is strongly coprime to $\mathcal{Q}$,

$$
\pi_{1}\left(J_{S}\right) \subset \pi_{1}(V) \subset \pi_{1}(Z)_{\mathcal{M}}^{(n+1)},
$$

and similarly for $\pi_{1}\left(D_{s}\right)$. This would complete the verification of claims (3) and (4) since $\left\{J_{s}, D_{s}\right\}$ would then satisfy the needed criteria of Definition 3.3. We will now turn our attention toward confirming claims (5) and (6). Let $G=\pi_{1}(Z)$. Let $\mu_{i}^{j}$ denote both the meridians of $L_{i}$ in $M_{L_{i}} \subset Z$ and its homotopy class in $G$. By abuse of notation, let $\eta_{i}^{j}$ also denote its push-off in $M_{L_{i}} \subset Z$ (the abuse is slight since these are isotopic in $E_{i}$ by Lemma 2.1). To prove claims (5) and (6) we will need the following lemma.

Lemma 5.3 For $0 \leq i \leq n$ and all $j$,

$$
\mu_{i}^{j} \in G^{(n-i)} \quad \text { and } \quad \eta_{i}^{j} \in G^{(n-i+1)},
$$

and if, for some $\ell, \eta_{\ell}^{j} \in G_{\mathcal{M}}^{(n-\ell+2)}$ for all $j$ then for each $i, 0 \leq i \leq \ell$,

$$
\mu_{i}^{j} \in G_{\mathcal{M}}^{(n-i+1)} \text { and } \eta_{i}^{j} \in G_{\mathcal{M}}^{(n-i+2)} .
$$


Proof We will establish the first clause of the lemma by reverse induction on $i$. For $i=n$, clearly $\mu_{n} \in G^{(0)} \equiv G$ and since, by definition of a doubling operator, $\eta_{n}$ lies in $\pi_{1}\left(M_{\mathcal{R}^{n}}\right)^{(1)}, \eta_{n} \in G^{(1)}$. This is the base of the induction. Now assume that $\mu_{i}^{j} \in G^{(n-i)}$ and $\eta_{i}^{j} \in G^{(n-i+1)}$. By property (1) of Lemma 2.1, $\mu_{i-1}^{j}$ is isotopic in $E_{i}$ to a push-off of $\eta_{i}^{j} \subset M_{L_{i}}$. It follows that

$$
\mu_{i-1}^{j} \in G^{(n-i+1)} .
$$

Since $\left\{\mu_{i-1}\right\}$ normally generates $\pi_{1}\left(M_{L_{i-1}}\right)$, it follows that $\pi_{1}\left(M_{L_{i-1}}\right) \subset G^{(n-i+1)}$. Thus,

$$
\eta_{i-1}^{j} \in\left[\pi_{1}\left(M_{L_{i-1}}\right), \pi_{1}\left(M_{L_{i-1}}\right)\right] \subset G^{(n-i+2)} .
$$

This completes the inductive step and establishes the first claim of the lemma.

Now suppose that $\eta_{\ell}^{j} \in G_{\mathcal{M}}^{(n-\ell+2)}$ for some $\ell$ and all $j$. By property (1) of Lemma 2.1, we have $\mu_{\ell-1}^{j}$ is isotopic to a push-off of $\eta_{\ell}^{j}$. Thus

$$
\mu_{\ell-1}^{j} \in G_{\mathcal{M}}^{(n-\ell+2)} .
$$

Then, as above, it follows that $\pi_{1}\left(M_{L_{\ell-1}}\right) \subset G_{\mathcal{M}}^{(n-\ell+2)}$, and so

$$
\eta_{\ell-1}^{j} \in\left[\pi_{1}\left(M_{L_{\ell-1}}\right), \pi_{1}\left(M_{L_{\ell-1}}\right)\right] \subset\left[G_{\mathcal{M}}^{(n-\ell+2)}, G_{\mathcal{M}}^{(n-\ell+2)}\right] \subset G_{\mathcal{M}}^{(n-\ell+3)}
$$

for all $j$, where for the last inclusion we use property (1) of Proposition 3.2. This establishes the second clause of the lemma in the case that $i=\ell-1$. Note that $\mu_{\ell-2}^{j}$ is isotopic to a push-off of $\eta_{\ell-1}^{j}$ and the argument iterates. This establishes the second clause.

Claims (5) and (3) follow immediately. We must now verify claim (6). Recall that by hypothesis $\mathcal{M}=\left(p_{1}, \ldots, p_{n}\right)$ is strongly coprime to $\mathcal{Q}=\left(q_{n}, \ldots, q_{1}\right)$. Hence there is some $\ell, 1 \leq \ell \leq n$, such that $p_{n-\ell+1}$ is strongly coprime to $q_{\ell}$. Note that $\eta_{\ell}^{j}$ is $q_{\ell}$ torsion in $\mathcal{A}\left(\mathcal{R}^{\ell}\right)$. Thus the push-off of $\eta_{\ell}^{j}$ is $q_{\ell}$ torsion in $\mathcal{A}\left(K_{\ell}\right)$. This can be interpreted in terms of the fundamental group of $M_{L_{\ell}}$ as follows [26, p. 174]. Suppose

$$
q_{\ell}\left(\mu_{\ell}^{1}, \ldots, \mu_{\ell}^{m}\right)=\sum_{m_{1}, \ldots, m_{r}} c_{m_{1}, \ldots, m_{r}}\left(\mu_{\ell}^{1}\right)^{m_{1}}\left(\mu_{\ell}^{2}\right)^{m_{2}}, \ldots,\left(\mu_{\ell}^{r}\right)^{m_{r}} .
$$

Then the fact that $\eta_{\ell}^{j}$ is $q_{\ell}$ torsion translates to the fact that

$\begin{aligned} \prod_{m_{1}, \ldots, m_{r}}\left(\mu_{\ell}^{1}\right)^{m_{1}}, \ldots,\left(\mu_{\ell}^{r}\right)^{m_{r}}\left(\eta_{\ell}^{i}\right)^{c_{m_{1}, \ldots, m_{r}}\left(\mu_{\ell}^{1}\right)^{-m_{1}}, \ldots,},\left(\mu_{\ell}^{r}\right)^{-m_{r}} & \\ & \in\left[\pi_{1}\left(M_{K_{\ell}}\right)^{(1)}, \pi_{1}\left(M_{K_{\ell}}\right)^{(1)}\right] .\end{aligned}$

Thus the right-hand expression is in

$$
\left[G^{(n-\ell+1)}, G^{(n-\ell+1)}\right] \subset\left[G_{\mathcal{M}}^{(n-\ell+1)}, G_{\mathcal{M}}^{(n-\ell+1)}\right]
$$


since we have shown in the proof of Lemma 5.3 that $\eta_{\ell}^{j} \in G^{(n-\ell+1)} \subset G_{\mathcal{M}}^{(n-\ell+1)}$. By Lemma 5.3, $\mu_{\ell}^{j} \in G_{\mathcal{M}}^{(n-\ell)}$, so $q_{\ell}\left(\mu_{\ell}^{1}, \ldots, \mu_{\ell}^{m}\right) \in \mathbb{Z}\left[G^{(n-\ell)} / G_{\mathcal{M}}^{(n-\ell+1)}\right]$. Therefore $\eta_{\ell}^{j}$ represents an element in the $\mathbb{Z}\left[G / G_{\mathcal{M}}^{(n-\ell+1)}\right]$-module $G_{\mathcal{M}}^{(n-\ell+1)} /\left[G_{\mathcal{M}}^{(n-\ell+1)}, G_{\mathcal{M}}^{(n-\ell+1)}\right]$, which is annihilated by $q_{\ell}\left(\mu_{\ell}^{1}, \ldots, \mu_{\ell}^{m}\right)$.

Since $p_{n-\ell+1}$ is strongly coprime to $q_{\ell}\left(\mu_{\ell}^{1}, \ldots, \mu_{\ell}^{m}\right)$ by Definition $4.7, q_{\ell} \in S_{p_{n-\ell+1}, A}^{\Gamma}$, where

$$
\Gamma=G / G_{\mathcal{M}}^{(n-\ell+1)} \text { and } A=G_{\mathcal{M}}^{(n-\ell)} / G_{\mathcal{M}}^{(n-\ell+1)} .
$$

Therefore, by Definition 4.10, $\eta_{\ell}^{j} \in G_{\mathcal{M}}^{(n-\ell+2)}$. Therefore, by the second clause of Lemma 5.3 (applied with $i=0$ ), $\mu_{0} \in G_{\mathcal{M}}^{(n+1)}$. This finishes the verification of claim (6), and hence the proof of the theorem.

Since $\mathfrak{m}_{k}$ (from Example 4.4) is strongly coprime to all single-variable polynomials there are specific examples of $\mathcal{M}$ that are strongly coprime to all $\mathcal{Q}$ composed of single-variable polynomials. Thus all knots obtained by iterated applications of knot doubling operators to a knot in $\mathcal{F}_{0}$ are trivial in $\mathcal{F}_{n} / \mathcal{F}_{n .5}^{\mathcal{M}}$.

\section{Distinguishing concordance classes: Nontriviality}

We will now show that there are knots resulting from an $n$-fold composition of string link doubling operators that are nontrivial in $\mathcal{F}_{n} / \mathcal{F}_{n .5}^{\mathcal{M}}$. Furthermore, by applying Theorem 5.2 it can be shown that these knots are distinct from any knots arising from certain compositions of knot doubling operators acting on a knot in $\mathcal{F}_{0}$. First, though, we will have to define the doubling operators that will be used.

Define $\mathfrak{R}_{\mathcal{D}}^{k}$ to be the doubling operator arising from the ribbon knot and properly embedded 1-multidisk shown in Figure 11. It is easy to show that $\eta$ is a generator of $\mathcal{A}\left(\mathfrak{R}^{k}\right)$. The Alexander polynomial of $\mathfrak{R}^{k}$ is $\mathfrak{p}_{k}$.

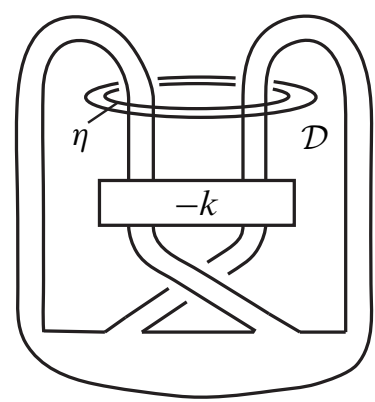

Figure 11: The doubling operator $\mathfrak{R}_{\mathcal{D}}^{k}$ 
Define $\left(\mathfrak{R}^{k} \# \mathfrak{R}^{\ell}\right)_{\mathcal{D}}$ to be the doubling operator arising from the ribbon knot and properly embedded 2-multidisk in Figure 12.

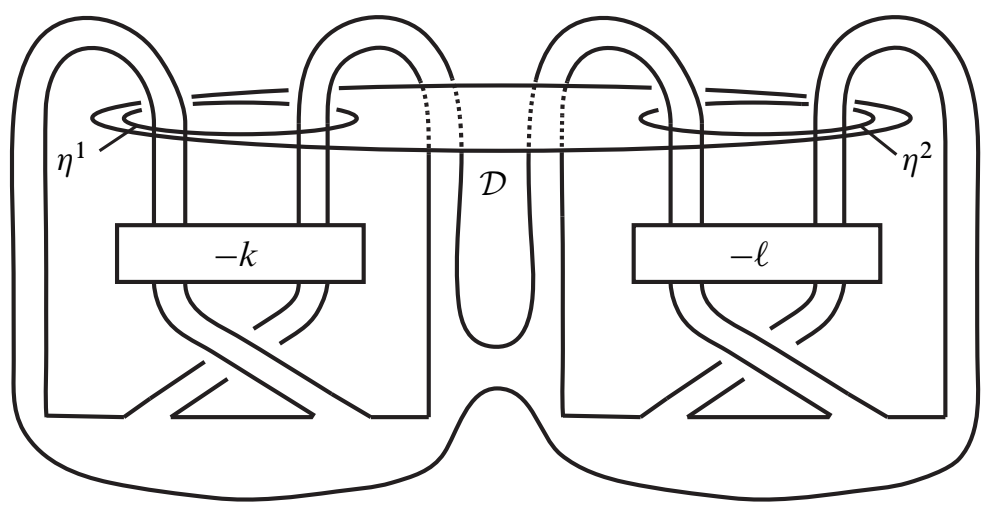

Figure 12: The doubling operator $\left(\mathfrak{R}^{k} \# \mathfrak{R}^{\ell}\right)_{\mathcal{D}}$

The $\eta^{i}$ form a basis for $\mathcal{A}\left(\mathfrak{R}^{k} \# \mathfrak{R}^{\ell}\right)$ since the Alexander module decomposes over connected sums. Since the Alexander polynomial is multiplicative under connected sum, the Alexander polynomial of the above knots is $\mathfrak{p}_{k} \mathfrak{p}_{\ell}$.

Define $\mathfrak{L}_{\mathcal{D}}^{k}$ to be a doubling operator arising from a slice string link shown in Figure 13 with properly embedded 1 -multidisks $\mathcal{D}=\mathcal{D}_{1} \sqcup \mathcal{D}_{2}$.

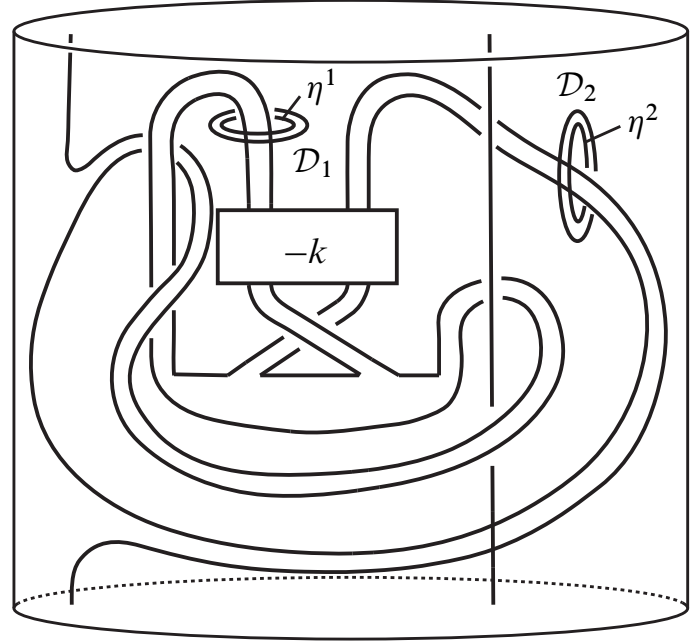

Figure 13: The doubling operator $\mathfrak{L}_{\mathcal{D}}^{k}$ 
The $\eta^{i}$ form a basis for $T \mathcal{A}\left(\widehat{\mathfrak{L}}^{k}\right)$, the torsion submodule of $\mathcal{A}\left(\hat{\mathfrak{L}}^{k}\right)$. As was shown before, the Alexander polynomial of $\hat{\mathfrak{L}}^{k}$ is $\mathfrak{m}_{k}$ with factors $\mathfrak{m}_{k}^{1}$ and $\mathfrak{m}_{k}^{2}$. Thus $\hat{\mathfrak{L}}^{k}$ has an Alexander polynomial that is strongly coprime to all single-variable polynomials by Lemma 4.5.

\subsection{Main theorems}

First we will describe a generalization of $(n, *)$-solutions called $(n, *)$-bordisms, which will be useful in the proof of the main result.

Definition 6.1 [10, Definition 7.11] For a given commutator series $*$, a compact spin smooth 4 -manifold $W$ is an $(n, *)$-bordism for $\partial W$ if:

(1) $H_{2}(W ; \mathbb{Z}) / H_{2}(\partial W ; \mathbb{Z})$ has a basis consisting of connected, compact, oriented surfaces, $\left\{J_{i}, D_{i} \mid 1 \leq i \leq r\right\}$, embedded in $W$ with trivial normal bundles, wherein the surfaces are pairwise disjoint except that, for each $i, J_{i}$ intersects $D_{i}$ transversely once with positive sign.

(2) For each $i, \pi_{1}\left(J_{i}\right) \subset \pi_{1}(W)_{*}^{(n)}$ and $\pi_{1}\left(D_{i}\right) \subset \pi_{1}(W)_{*}^{(n)}$.

Additionally, $W$ is an $(n .5, *)$-bordism if:

(3) For each $i, \pi_{1}\left(J_{i}\right) \subset \pi_{1}(W)_{*}^{(n+1)}$.

An $(n, *)$-solution is an $(n, *)$-bordism, but $(n, *)$-bordisms need not have connected boundary and the inclusion map from the boundary does not necessarily induce an isomorphism on $H_{1}$.

Theorem 6.2 Fix some $n \geq 2$. Let

$$
K_{0} \in \mathcal{F}_{0} \quad \text { and } \quad K_{n-2}=\mathcal{R}_{\mathcal{D}_{n-2}}^{n-2} \circ \cdots \circ \mathcal{R}_{\mathcal{D}_{1}}^{1}\left(K_{0}\right),
$$

where $\mathcal{R}_{\mathcal{D}_{i}}^{i}$, for $1 \leq i \leq n-2$, is $\Re_{\mathcal{T}}^{k_{i}}$ for some $k_{i} \geq 1$. Let

$$
L_{n-1}=\mathcal{R}_{\mathcal{D}_{n-1}}^{n-1}\left(K_{n-2}, K_{n-2}\right),
$$

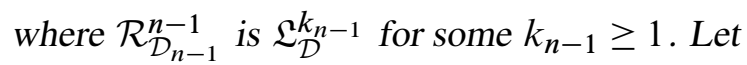

$$
K=K_{n}=\mathcal{R}_{\mathcal{D}_{n}}^{n}\left(L_{n-1}\right),
$$

where $\mathcal{R}_{\mathcal{D}_{n}}^{n}$ is $\left(\mathfrak{R}^{1} \# \mathfrak{R}^{1}\right)_{\mathcal{D}}$. Let $\mathcal{M}=\left(\mathfrak{p}_{1} \mathfrak{p}_{2}, \mathfrak{m}_{k_{n-1}}, \mathfrak{p}_{k_{n-2}}, \ldots, \mathfrak{p}_{k_{1}}\right)$. If $K_{0}$ is chosen so that

$$
\left|\rho_{0}\left(M_{K_{0}}\right)\right|>C_{\mathcal{R}^{n}}+C_{\widehat{\mathcal{R}}^{n-1}}+2\left(C_{\mathcal{R}^{n-2}} \cdots+C_{\mathcal{R}^{1}}\right)+3,
$$

where $C_{\mathcal{R}^{j}}$ is the Cheeger-Gromov constant for $M_{\mathcal{R}^{j}}$, then $K$ is of infinite order in $\mathcal{F}_{n} / \mathcal{F}_{n .5}^{\mathcal{M}}$. 
Note that there do exist knots $K_{0}$ satisfying the needed $\rho_{0}$ condition. Since $\rho_{0}$ is additive under connected sum and the trefoil has nontrivial $\rho_{0}$, one can create an Arf invariant zero knot with arbitrarily large $\rho_{0}$ by taking a connected sum of trefoils. One would need a connected sum of an even number of trefoils in order to obtain a knot with Arf invariant zero.

The following proof is a modified version of the proof given in $[10$, Section 7 , Theorem 7.5], but here we will incorporate doubling operators arising from slice string links and the newly defined derived series localized at $\mathcal{M}$.

Proof First, note that by Corollary 3.8 and Proposition 3.9, $K \in \mathcal{F}_{n} \subset \mathcal{F}_{n}^{\mathcal{M}}$ and thus $m K \in \mathcal{F}_{n}^{\mathcal{M}}$ for $m \geq 1$ where $m K$ is the $m$-fold connected sum of $K$ with itself. Therefore it remains to show that $m K \notin \mathcal{F}_{n .5}^{\mathcal{M}}$. We proceed by contradiction. Suppose that $m K \in \mathcal{F}_{n .5}^{\mathcal{M}}$. Then there is an $(n .5, \mathcal{M})$-solution $V$ for $m K$.

Since $m K$ can be viewed as arising by infecting $K$ with $K$ along $(m-1) 1-$ multidisks, there is a cobordism $C$ (see Section 3.1) with boundary $M_{m K} \sqcup M_{n}^{1} \sqcup \cdots \sqcup M_{n}^{m}$ (where we will abbreviate the zero-framed surgery of $K_{i}$ by $M_{i}$ ). The cobordism $E$ from Section 2.1 can be modified to incorporate infections at multiple multidisks. Note that any of the copies of $K$ can be viewed as the infected knot. In this proof we will refer to properties of $E$ that are true for this more general case, which includes $C$. The integral homology of $C$ was analyzed in [12, pages 113-114]. Consider $C \cup_{M_{m K}} V$ (see Figure 14). We will prove some facts about the homology of $C \cup V$. We start by showing that $H_{1}(C \cup V) \cong H_{1}(C) \cong H_{1}\left(M_{n}^{i}\right)$. Consider the following Mayer-Vietoris sequence:

$$
H_{1}\left(M_{m K}\right) \rightarrow H_{1}(C) \oplus H_{1}(V) \rightarrow H_{1}(C \cup V) \rightarrow 0
$$

Since $V$ is an $(n, \mathcal{M})$-solution for $m K, H_{1}(V)$ is isomorphic to $H_{1}\left(M_{m K}\right)$ and is generated by the meridian of $m K$. Therefore, $H_{1}(C \cup V) \cong H_{1}(C) \cong H_{1}\left(M_{n}^{i}\right)$, where the last inequality is by property (3) of Lemma 2.1 .

We will now analyze $H_{2}(C \cup V)$. We start by showing that $H_{3}(C \cup V, V)=0$. Note that by excision $H_{3}(C \cup V, V) \cong H_{3}\left(C, M_{m K}\right) \cong H_{1}\left(C, M_{n}^{1} \sqcup \cdots \sqcup M_{n}^{m}\right)$, where the last isomorphism is from Poincaré duality. Consider the long exact sequence of the pair

$$
H_{1}\left(M_{n}^{1}\right) \oplus \cdots \oplus H_{1}\left(M_{n}^{m}\right) \rightarrow H_{1}(C) \rightarrow H_{1}\left(C, M_{n}^{1} \sqcup \cdots \sqcup M_{n}^{m}\right) \rightarrow 0 .
$$

By property (3) of Lemma 2.1 we can infer that the leftmost map is a surjection, which implies that the middle is the zero map. Therefore

$$
H_{3}(C \cup V, V) \cong H_{1}\left(C, M_{n}^{1} \sqcup \cdots \sqcup M_{n}^{m}\right)=0 .
$$


From this observation we obtain the long exact sequence of the pair

$$
0 \rightarrow H_{2}(V) \rightarrow H_{2}(C \cup V) \rightarrow H_{2}(C \cup V, V) \rightarrow H_{1}(V) \rightarrow H_{1}(C \cup V) .
$$

By property (3) of Lemma 2.1, we know that the map induced by inclusion,

$$
H_{1}\left(M_{m K}\right) \rightarrow H_{1}(C),
$$

is an isomorphism, and by examining the Mayer-Vietoris sequence associated to the union of $V$ and $C$, we can show that the map induced by inclusion, $H_{1}(V) \rightarrow$ $H_{1}(C \cup V)$, is an isomorphism. Therefore the last map of (7) is an isomorphism and thus the map that precedes it is the zero map. We can then infer that the third map is a surjection. Putting this together we see that we have the split short exact sequence

$$
0 \rightarrow H_{2}(V) \rightarrow H_{2}(C \cup V) \rightarrow H_{2}(C \cup V, V) \rightarrow 0 .
$$

Therefore

$$
H_{2}(C \cup V) \cong H_{2}(V) \oplus H_{2}(C \cup V, V) .
$$

By excision $H_{2}(C \cup V, V) \cong H_{2}\left(C, M_{m K}\right)$. Consider the exact sequence of a pair

$$
H_{2}\left(M_{m K}\right) \rightarrow H_{2}(C) \rightarrow H_{2}\left(C, M_{m K}\right) \rightarrow H_{1}\left(M_{m K}\right) \rightarrow H_{1}(C) .
$$

Again by property (3) of Lemma 2.1, we know that last map of the sequence is an isomorphism and thus the second to last map is the zero map. By property (4) of Lemma 2.1, we know that $H_{2}(C) \cong H_{2}\left(M_{K^{m}}\right) \oplus H_{2}\left(M_{n}^{2} \sqcup \cdots \sqcup M_{n}^{m}\right)$. We can infer that

$$
H_{2}\left(C, M_{m K}\right) \cong H_{2}\left(M_{n}^{2} \sqcup \cdots \sqcup M_{n}^{m}\right)
$$

Therefore

$$
H_{2}(C \cup V) \cong H_{2}(V) \oplus H_{2}\left(M_{n}^{2} \sqcup \cdots \sqcup M_{n}^{m}\right) .
$$

Let $j_{*}^{\prime}: H_{2}(\partial V) \rightarrow H_{2}(C \cup V)$ be the map induced by the inclusion map $j^{\prime}: \partial V \rightarrow$ $C \cup V$. Likewise, let $j_{*}^{\prime \prime}: H_{2}(\partial(C \cup V)) \rightarrow H_{2}(C \cup V)$ be the map induced by the inclusion map $j^{\prime \prime}: \partial(C \cup V) \rightarrow C \cup V$. Note that $\partial V=M_{m K}$ and $\partial(C \cup V)=M_{m K} \sqcup$ $M_{n}^{1} \sqcup \cdots \sqcup M_{n}^{m}$. By the proof of property (3) of Lemma 2.1 we know that $H_{2}\left(M_{m K}\right)$ is isomorphic to $H_{2}\left(M_{K}\right)$ via inclusion into $C$ and thus inclusion into $C \cup V$. From this we can infer that $j_{*}^{\prime \prime}\left(H_{2}(\partial C \cup V)\right) \cong j_{*}^{\prime}\left(H_{2}(\partial V)\right) \oplus j_{*}^{\prime \prime}\left(H_{2}\left(M_{n}^{2}\right) \oplus \cdots \oplus H_{2}\left(M_{n}^{m}\right)\right)$. Therefore,

$$
\begin{aligned}
& \frac{H_{2}(C \cup V)}{j_{*}^{\prime \prime}\left(H_{2}(\partial(C \cup V))\right)} \cong \frac{H_{2}(V) \oplus H_{2}\left(M_{n}^{2}\right) \oplus \cdots \oplus H_{2}\left(M_{n}^{m}\right)}{j_{*}^{\prime}\left(H_{2}(\partial V)\right) \oplus j_{*}^{\prime \prime}\left(H_{2}\left(M_{n}^{2}\right) \oplus \cdots \oplus H_{2}\left(M_{n}^{m}\right)\right)} \\
& \cong \frac{H_{2}(V)}{j_{*}^{\prime}\left(H_{2}(\partial V)\right)} .
\end{aligned}
$$


Let $C \cup V$ be called $W_{0}$ (see Figure 14). By the naturality of the intersection form with twisted coefficients, it then follows that the same surfaces used to show that $V$ is a $(n, \mathcal{M})$-bordism can be used to show that $W_{0}$ is a $(n, \mathcal{M})$-bordism since if $J$ is such a surface in $V$ and $\pi_{1}(J) \subset \pi_{1}(V)_{\mathcal{M}}^{(n)}$. Then $\pi_{1}(J) \subset \pi_{1}\left(W_{0}\right)_{\mathcal{M}}^{(n)}$ by the functorality of the derived series localized at $\mathcal{M}$.

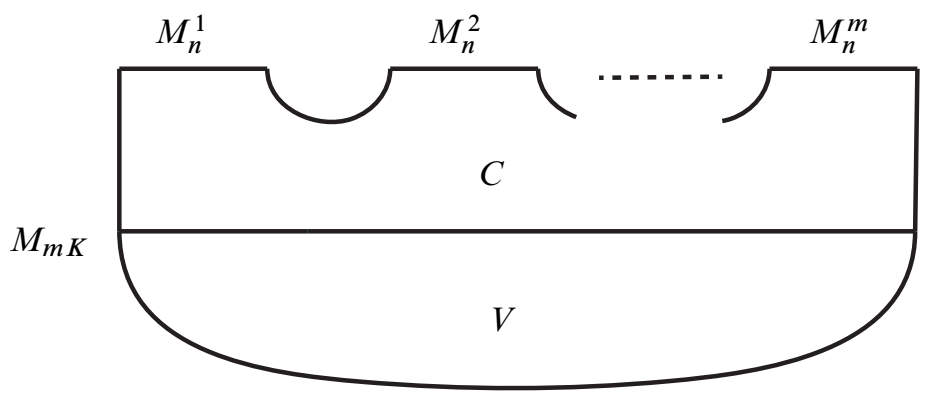

Figure 14: The $(n, \mathcal{M})$-bordism $W_{0}$

Stage 0 Define $j_{0}^{j}: M_{n}^{j} \rightarrow W_{0}$, for $j=1, \ldots, m$, to be the inclusion map. From above we know that $j_{0 *}^{j}: H_{1}\left(M_{n}^{j} ; \mathbb{Q}\right) \rightarrow H_{1}\left(W_{0} ; \mathbb{Q}\right)$ is an isomorphism. Therefore, $j_{0 *}^{j}\left(\pi_{1}\left(M_{n}^{j}\right)\right) \cong \mathbb{Z} \subset \pi_{1}\left(W_{0}\right) / \pi_{1}\left(W_{0}\right)_{\mathcal{M}}^{(1)}$.

We now want to show $\eta_{n}^{j, 1}, \eta_{n}^{j, 2}$ of $\mathcal{D}_{n}^{j}$ are nontrivial in $\pi_{1}\left(W_{0}\right)^{(1)} / \pi_{1}\left(W_{0}\right)_{\mathcal{M}}^{(2)}$ and, furthermore, that $\eta_{n}^{j, 1}$ and $\eta_{n}^{j, 2}$ are linearly independent in $\pi_{1}\left(W_{0}\right)^{(1)} / \pi_{1}\left(W_{0}\right)_{\mathcal{M}}^{(2)}$. Note that we have $\eta_{n}^{j, 1}, \eta_{n}^{j, 2} \in \pi_{1}\left(M_{n}^{j}\right)^{(1)} \subset \pi_{1}\left(W_{0}\right)^{(1)}$.

Let $\Lambda_{0}=\pi_{1}\left(W_{0}\right) / \pi_{1}\left(W_{0}\right)_{\mathcal{M}}^{(1)} \cong \mathbb{Z}$ and let $\psi_{0}: \pi_{1}\left(W_{0}\right) \rightarrow \Lambda_{0}$ be the canonical surjection. Define $R_{0}=\left(\mathbb{Q} \Lambda_{0}\right) S_{\mathfrak{p}_{1}}^{-1} \cong\left(\mathbb{Q}\left[t, t^{-1}\right]\right) S_{\mathfrak{p}_{1}}^{-1}$. Note that

$$
H_{1}\left(W_{0} ; R_{0}\right) \cong\left(\pi_{1}\left(W_{0}\right)^{(1)} /\left[\pi_{1}\left(W_{0}\right)^{(1)}, \pi_{1}\left(W_{0}\right)^{(1)}\right]\right) \otimes_{\mathbb{Z} \Lambda_{0}} R_{0} .
$$

Consider the following commutative diagram:

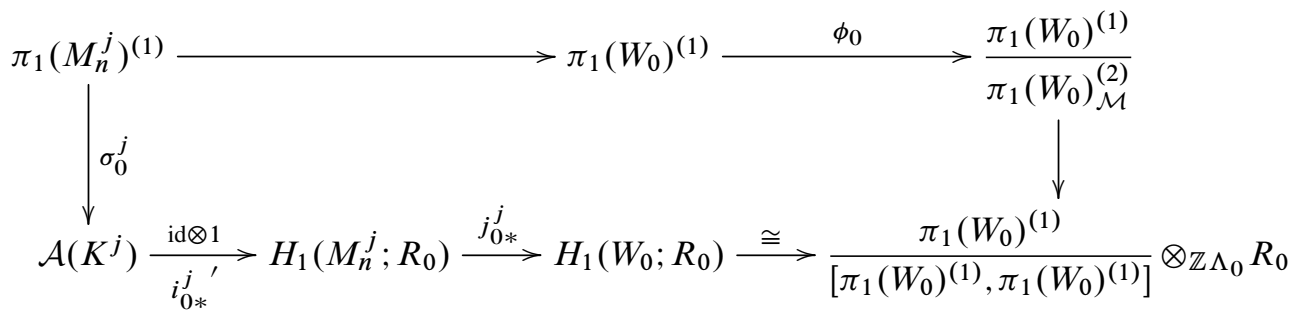

Since the right-hand vertical map is injective by the definition of the group localized at $M$, if $\eta_{n}^{j, i}$ is in the kernel of the top row, $\eta_{n}^{j, i} \in \mathcal{A}\left(K^{j}\right)$ must be in the kernel 
of the bottom row. If this were true then $\eta_{n}^{j, i} \otimes 1$ would be in the kernel of $j_{0 *}^{j}$. By [10, Theorem 7.15], ker $j_{0 *}^{j}=P_{0}^{j}$ is an isotropic submodule $\left(P_{0}^{j} \subset P_{0}^{j \perp}\right)$ with respect to the Blanchfield linking form on $H_{1}\left(M_{n}^{j} ; R_{0}\right)$.

The Alexander module and Blanchfield linking form of $K^{j}$ and $\mathfrak{R}^{1} \# \mathfrak{R}^{1}$ are isomorphic since $K^{j}$ is obtained by infection on $\mathfrak{R}^{1} \# \mathfrak{R}^{1}$ (Cochran [5, Theorem 8.1], Leidy [21, Theorem 4.1]). Furthermore, the Blanchfield linking form decomposes over the connected sum. Therefore,

$$
\mathcal{B} \ell_{R_{0}}^{K^{j}}\left(\eta_{n}^{j, i} \otimes 1, \eta_{n}^{j, i} \otimes 1\right)=\mathcal{B} \ell_{R_{0}}^{\Re^{1}}\left(\eta_{n}^{j, i} \otimes 1, \eta_{n}^{j, i} \otimes 1\right) .
$$

From Leidy [20, Proposition 3.6; 21, Theorem 4.7], we have

$$
\mathcal{B} \ell_{R_{0}}^{\Re^{1}}\left(\eta_{n}^{j, i} \otimes 1, \eta_{n}^{j, i} \otimes 1\right)=\bar{\phi}\left(\mathcal{B} \ell_{0}^{\Re^{1}}\left(\eta_{n}^{j, i}, \eta_{n}^{j, i}\right)\right),
$$

where $\mathcal{B} \ell_{0}^{\mathfrak{R}^{1}}$ is the classical Blanchfield linking form and $\bar{\phi}$ is the map

$$
\bar{\phi}: \frac{\mathbb{Q}(t)}{\mathbb{Q}\left[t, t^{-1}\right]} \rightarrow \frac{\mathbb{Q}(t)}{\mathbb{Q}\left[t, t^{-1}\right] S_{\mathfrak{p}_{1}}^{-1}} .
$$

One can calculate $\mathcal{B} \ell_{0}^{\mathfrak{R}^{1}}\left(\eta_{n}^{j, i}, \eta_{n}^{j, i}\right)$ and obtain

$$
\mathcal{B} \ell_{0}^{\Re^{1}}\left(\eta_{n}^{j, i}, \eta_{n}^{j, i}\right)=\frac{3 t^{2}-6 t+3}{2 t^{2}-5 t+2}=\frac{3}{2}+\frac{\frac{3}{2} t}{2 t^{2}-5 t+2} .
$$

We can then calculate $\bar{\phi}\left(\mathcal{B} \ell_{0}^{\mathfrak{R}^{1}}\left(\eta_{n}^{j, i}, \eta_{n}^{j, i}\right)\right)$. Note $2 t^{2}-5 t+2$ is $\mathfrak{p}_{1}$. Thus by the definition of $S_{\mathfrak{p}_{1}}^{-1}$, we see that

$\mathcal{B} \ell_{R_{0}}^{\mathfrak{R}^{1}}\left(\eta_{n}^{j, i} \otimes 1, \eta_{n}^{j, i} \otimes 1\right)=\bar{\phi}\left(\mathcal{B} \ell_{0}^{\mathfrak{R}^{1}}\left(\eta_{n}^{j, i}, \eta_{n}^{j, i}\right)\right)=\bar{\phi}\left(\frac{3 t^{2}-6 t+3}{2 t^{2}-5 t+2}\right)=\frac{\frac{3}{2} t}{2 t^{2}-5 t+2} \neq 0$.

Therefore, $\eta_{n}^{j, i} \notin P_{0}^{j}$ and $\eta_{n}^{j, i}$ is nontrivial in $\pi_{1}\left(W_{0}\right)^{(1)} / \pi_{1}\left(W_{0}\right)_{\mathcal{M}}^{(2)}$.

Using the same machinery, we can show, in fact, that $\eta_{n}^{j, 1}$ and $\eta_{n}^{j, 2}$ are linearly independent in $\pi_{1}\left(W_{0}\right)^{(1)} / \pi_{1}\left(W_{0}\right)_{\mathcal{M}}^{(2)}$. Let $m_{1} \eta_{n}^{j, 1}+m_{2} \eta_{n}^{j, 2}$ be an arbitrary $\mathbb{Z}-$ linear combination where $m_{1} \neq 0$ and $m_{2} \neq 0$. (Since $\pi_{1}\left(W_{0}\right)^{(1)} / \pi_{1}\left(W_{0}\right)_{\mathcal{M}}^{(2)}$ is torsion-free abelian we are discussing $\mathbb{Z}$-linear combinations.) We need to show that $\left(\eta_{n}^{j, 1}\right)^{m_{1}}\left(\eta_{n}^{j, 2}\right)^{m_{2}} \in \pi_{1}\left(M_{n}^{j}\right)^{(1)} \subset \pi_{1}\left(W_{0}\right)^{(1)}$ is not in $\pi_{1}\left(W_{0}\right)_{\mathcal{M}}^{(2)}$.

We will show that $\left(\eta_{n}^{j, 1}\right)^{m_{1}}\left(\eta_{n}^{j, 2}\right)^{m_{2}} \notin \pi_{1}\left(W_{0}\right)_{\mathcal{M}}^{(2)}$ by showing that

$$
\sigma_{0}^{j}\left(\left(\eta_{n}^{j, 1}\right)^{m_{1}}\left(\eta_{n}^{j, 2}\right)^{m_{2}}\right)=m_{1} \eta_{n}^{j, 1}+m_{2} \eta_{n}^{j, 2} \in \mathcal{A}_{0}\left(K^{j}\right)
$$


is not in the kernel of the bottom row. If $m_{1} \eta_{n}^{j, 1}+m_{2} \eta_{n}^{j, 2}$ were in the kernel of the bottom row, then $m_{1} \eta_{n}^{j, 1} \otimes 1+m_{2} \eta_{n}^{j, 2} \otimes 1 \in P_{0}^{j}$, which implies

$$
\mathcal{B} \ell_{R_{0}}^{K^{j}}\left(m_{1} \eta_{n}^{j, 1} \otimes 1+m_{2} \eta_{n}^{j, 2} \otimes 1, m_{1} \eta_{n}^{j, 1} \otimes 1+m_{2} \eta_{n}^{j, 2} \otimes 1\right)=0 .
$$

Since $\mathcal{B} \ell_{R_{0}}^{K^{j}}$ decomposes over the connected sum,

$$
\begin{aligned}
\mathcal{B} \ell_{R_{0}}^{K^{j}}\left(m_{1} \eta_{n}^{j, 1} \otimes 1+\right. & \left.m_{2} \eta_{n}^{j, 2} \otimes 1, m_{1} \eta_{n}^{j, 1} \otimes 1+m_{2} \eta_{n}^{j, 2} \otimes 1\right) \\
& =m_{1}^{2} \mathcal{B} \ell_{R_{0}}^{\mathfrak{R}^{1}}\left(\eta_{n}^{j, 1} \otimes 1, \eta_{n}^{j, 1} \otimes 1\right)+m_{2}^{2} \mathcal{B} \ell_{\boldsymbol{R}_{0}}^{\mathfrak{R}^{1}}\left(\eta_{n}^{j, 2} \otimes 1, \eta_{n}^{j, 2} \otimes 1\right) .
\end{aligned}
$$

From the work above we can infer that

$$
\mathcal{B} \ell_{R_{0}}^{K^{j}}\left(m_{1} \eta_{n}^{j, 1} \otimes 1+m_{2} \eta_{n}^{j, 2} \otimes 1, m_{1} \eta_{n}^{j, 1} \otimes 1+m_{2} \eta_{n}^{j, 2} \otimes 1\right) \neq 0 .
$$

Thus $\eta_{n}^{j, 1}$ and $\eta_{n}^{j, 2}$ are linearly independent in $\pi_{1}\left(W_{0}\right)^{(1)} / \pi_{1}\left(W_{0}\right)_{\mathcal{M}}^{(2)}$.

Stage 1 Recall that there is a cobordism $E_{n}^{j}$ with boundary $M_{n}^{j} \sqcup M_{\mathcal{R}^{n}}^{j} \sqcup M_{n-1}^{j}$. Let

$$
W_{1}=\left(\bigsqcup_{j} E_{n}^{j}\right) \cup W_{0},
$$

where the union is along their common boundaries $M_{n}^{j}$. We wish to demonstrate that under the inclusion map $j_{1}^{j}: M_{n-1}^{j} \rightarrow W_{1}$,

$$
j_{1 *}^{j}\left(\pi_{1}\left(M_{n-1}\right)\right) \cong \mathbb{Z} \oplus \mathbb{Z} \subset \pi_{1}\left(W_{1}\right)^{(1)} / \pi_{1}\left(W_{1}\right)_{\mathcal{M}}^{(2)} .
$$

By part (2) of Lemma 2.1, $\eta_{n}^{j, 1}$ and $\eta_{n}^{j, 2}$ are isotopic in $E_{n}^{j}$ to the meridians of $L_{n-1}^{j}$. We therefore will also refer to the meridians of $L_{n-1}^{j}$ as $\eta_{n}^{j, 1}$ and $\eta_{n}^{j, 2}$. Recall that $\pi_{1}\left(M_{n-1}^{j}\right)$ is normally generated by $\left\{\eta_{n}^{j, 1}, \eta_{n}^{j, 2}\right\}$, and $\eta_{n}^{j, 1}$ and $\eta_{n}^{j, 2}$ are linearly independent in $\pi_{1}\left(W_{0}\right)^{(1)} / \pi_{1}\left(W_{0}\right)_{\mathcal{M}}^{(2)}$. Therefore (9) can be demonstrated by showing that

$$
\pi_{1}\left(W_{0}\right)^{(1)} / \pi_{1}\left(W_{0}\right)_{\mathcal{M}}^{(2)} \cong \pi_{1}\left(W_{1}\right)^{(1)} / \pi_{1}\left(W_{1}\right)_{\mathcal{M}}^{(2)} .
$$

By multiple applications of part (2) of Lemma 2.1, the map

$$
\pi_{1}\left(W_{0}\right) \rightarrow \pi_{1}\left(W_{0} \cup_{\bigsqcup_{j} M_{n}^{j}} E_{n}^{j}\right)=\pi_{1}\left(W_{1}\right),
$$

induced by the inclusion map, is a surjection whose kernel is the normal closure of the longitudes, $\ell_{n-1}^{j, i}$, of $L_{n-1}^{j}$. We assume that $\eta_{n}^{j, i}$ is the meridian of the component of $L_{n-1}^{j}$ with $\ell_{n-1}^{j, i}$ as its longitude. Thus, since $\eta_{n}^{j, i} \in \pi_{1}\left(W_{0}\right)^{(1)}, \ell_{n-1}^{j, i} \in \pi_{1}\left(W_{0}\right)^{(2)} \subset$ $\pi_{1}\left(W_{0}\right)_{\mathcal{M}}^{(2)}$. Therefore by Proposition 4.12, (10) is affirmed. 
We now demonstrate $W_{1}$ is an $(n, \mathcal{M})$-bordism. We know $W_{0}$ is a $(n, \mathcal{M})$-bordism. Since $W_{1}$ is created from $W_{0}$ by adding $\bigsqcup_{j} E_{n}^{j}$ where $H_{2}\left(E_{n}^{j}\right) / H_{2}\left(\partial E_{n}^{j}\right)=0$, as when analyzing $\mathrm{H}_{2}(C \cup V)$, a series of long exact sequences arguments shows that

$$
H_{2}\left(W_{0}\right) / H_{2}\left(\partial W_{0}\right) \cong H_{2}\left(W_{1}\right) / H_{2}\left(\partial W_{1}\right)
$$

It then follows that the same surfaces used to show that $W_{0}$ is an $(n, \mathcal{M})$-bordism can be used to show that $W_{1}$ is an $(n, \mathcal{M})$-bordism since if $J$ is such a surface and $\pi_{1}(J) \subset \pi_{1}\left(W_{0}\right)_{\mathcal{M}}^{(n)}$, then $\pi_{1}(J) \subset \pi_{1}\left(W_{1}\right)_{\mathcal{M}}^{(n)}$ by the functorality of the derived series localized at $\mathcal{M}$.

Let $\eta_{n-1}^{j, 1} \in \mathcal{D}_{n-1}^{1}$ and $\eta_{n-1}^{j, 2} \in \mathcal{D}_{n-1}^{2}$ as in Figure 13 . We want to show that $\eta_{n-1}^{j, 1}$ or $\eta_{n-1}^{j, 2} \in \pi_{1}\left(M_{n-1}\right)$ (or both) is nontrivial in $\pi_{1}\left(W_{1}\right)^{(2)} / \pi_{1}\left(W_{1}\right)_{\mathcal{M}}^{(3)}$ for each $j$. Note that $\eta_{n-1}^{j, i} \in \pi_{1}\left(M_{n-1}\right)^{(1)}$. Since $j_{1 *}^{j}\left(\pi_{1}\left(M_{n-1}\right)^{(1)}\right) \subset \pi_{1}\left(W_{1}\right)^{(2)}, \eta_{n-1}^{j, i} \in \pi_{1}\left(W_{1}\right)^{(2)}$.

Let $\Lambda_{1}=\pi_{1}\left(W_{1}\right) / \pi_{1}\left(W_{1}\right)_{\mathcal{M}}^{(2)}$ and let $\psi_{1}: \pi_{1}\left(W_{1}\right) \rightarrow \Lambda_{1}$ be the canonical surjection. Define $R_{1}=\left(\mathbb{Q} \Lambda_{1}\right) S_{\mathfrak{m}_{k-1}}^{-1}$. Note

$$
H_{1}\left(W_{1} ; R_{1}\right) \cong \pi_{1}\left(W_{1}\right)_{\mathcal{M}}^{(2)} /\left[\pi_{1}\left(W_{1}\right)_{\mathcal{M}}^{(2)}, \pi_{1}\left(W_{1}\right)_{\mathcal{M}}^{(2)}\right] \otimes_{\mathbb{Z} \Lambda_{1}} R_{1}
$$

Consider the following commutative diagram:

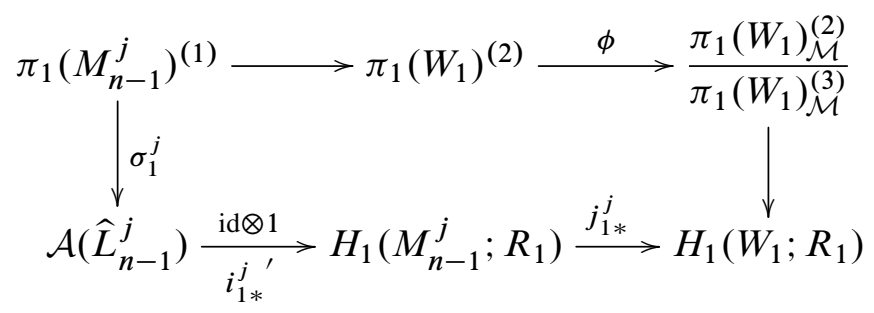

If $\eta_{n-1}^{j, i}$ is in the kernel of the top row, then $\eta_{n-1}^{j, i} \in \mathcal{A}\left(\hat{L}_{n-1}^{j}\right)$ is in the kernel of the bottom row. If this were true, then $\eta_{n-1}^{j, i} \otimes 1$ would be in the kernel of $j_{1 *}^{j}$. We will show that at least one of $\eta_{n-1}^{j, 1} \otimes 1$ and $\eta_{n-1}^{j, 2} \otimes 1$ is not $\operatorname{in} \operatorname{ker}\left(j_{1 *}^{j}\right)=P_{1}^{j}$.

It can be shown that

$$
\mathcal{A}\left(\hat{L}_{n-1}^{j}\right) \cong \mathcal{A}\left(\widehat{\mathcal{R}}_{j}^{n-1}\right) \cong \mathbb{Q}\left[t_{1}^{ \pm 1}, t_{2}^{ \pm 2}\right] \oplus \frac{\mathbb{Q}\left[t_{1}^{ \pm 1}, t_{2}^{ \pm 2}\right]}{\left\langle\mathfrak{m}_{k_{n-1}}^{1}\left(t_{1}, t_{2}\right)\right\rangle} \oplus \frac{\mathbb{Q}\left[t_{1}^{ \pm 1}, t_{2}^{ \pm 2}\right]}{\left\langle\mathfrak{m}_{k_{n-1}}^{2}\left(t_{1}, t_{2}\right)\right\rangle}
$$

The torsion submodule is nontrivial since $\mathfrak{m}_{k_{n-1}}^{i}\left(t_{1}, t_{2}\right)$, for $i=1,2$, is not a unit in $\mathbb{Q}\left[t_{1}^{ \pm 1}, t_{2}^{ \pm 2}\right]$. Thus,

$\mathcal{A}\left(\hat{L}_{n-1}^{j}\right) \otimes_{\mathbb{Q}\left[t_{1}^{ \pm 1}, t_{2}^{ \pm 2}\right]} \mathbb{Q} \Lambda_{1} \cong \mathbb{Q} \Lambda_{1} \oplus \frac{\mathbb{Q} \Lambda_{1}}{\mathfrak{m}_{k_{n-1}}^{1}\left(\eta_{n}^{j, 1}, \eta_{n}^{j, 2}\right) \mathbb{Q} \Lambda_{1}} \oplus \frac{\mathbb{Q} \Lambda_{1}}{\mathfrak{m}_{k_{n-1}}^{2}\left(\eta_{n}^{j, 1}, \eta_{n}^{j, 2}\right) \mathbb{Q} \Lambda_{1}}$ 
is a nontrivial module whose torsion summands are generated by $\eta_{n-1}^{j, 1}$ and $\eta_{n-1}^{j, 2}$ respectively, and where $\eta_{n-1}^{j, 1}$ and $\eta_{n-1}^{j, 2}$ are the classes in $\Lambda_{1}$ represented by the meridians of $\widehat{L}_{n-1}^{j}$ in $M_{n-1}^{j}$. From Step 1 we know that $\eta_{n-1}^{j, 1}$ and $\eta_{n-1}^{j, 2}$ are elements of infinite order and $\left\{\eta_{n}^{j, 1}, \eta_{n}^{j, 2}\right\}$ is linearly independent in $\pi_{1}\left(W_{1}\right)^{(1)} / \pi_{1}\left(W_{1}\right)_{\mathcal{M}}^{(2)}$. From this we can infer that

$$
\begin{aligned}
H_{1} & \left(M_{n-1}^{j} ; R_{1}\right) \\
& \cong \mathcal{A}\left(\widehat{L}_{n-1}^{j}\right) \otimes_{\mathbb{Q}\left[t_{1}^{ \pm 1}, t_{2}^{ \pm 2}\right]} R_{1} \\
& \cong \mathbb{Q} \Lambda_{1} S_{\mathfrak{m}_{k_{n-1}}}^{-1} \oplus \frac{\mathbb{Q} \Lambda}{\mathfrak{m}_{k_{n-1}}^{1}\left(\eta_{n}^{j, 1}, \eta_{n}^{j, 2}\right) \mathbb{Q} \Lambda} S_{\mathfrak{m}_{k_{n-1}}}^{-1} \oplus \frac{\mathbb{Q} \Lambda_{1}}{\mathfrak{m}_{k_{n-1}}^{2}\left(\eta_{n}^{j, 1}, \eta_{n}^{j, 2}\right) \mathbb{Q} \Lambda_{1}} S_{\mathfrak{m}_{k_{n-1}}}^{-1} \\
& \cong R_{1} \oplus \frac{R_{1}}{\mathfrak{m}_{k_{n-1}}^{1}\left(\eta_{n}^{j, 1}, \eta_{n}^{j, 2}\right) R_{1}} \oplus \frac{R_{1}}{\mathfrak{m}_{k_{n-1}}^{2}\left(\eta_{n}^{j, 1}, \eta_{n}^{j, 2}\right) R_{1}},
\end{aligned}
$$

an $R_{1}$-module whose torsion summands are generated by $\eta_{n-1}^{j, 1} \otimes 1$ and $\eta_{n-1}^{j, 2} \otimes 1$ respectively.

Lemma 6.3 We have $\operatorname{rank}_{\mathbb{Z} \Lambda_{1}} H_{1}\left(M_{n-1}^{j} ; \mathbb{Z} \Lambda_{1}\right)=\beta_{1}\left(M_{n-1}^{j}\right)-1$.

Proof First, note that $\widehat{L}_{n-1}^{j}$ is a boundary link and thus $H_{2}\left(M_{n-1}^{j} ; \mathbb{Q}\right)$ is generated by two disjoint capped off Seifert surfaces. These Seifert surfaces are displayed in Figure 15. Any loop on the Seifert surfaces lies in $\pi_{1}\left(M_{n-1}^{j}\right)^{(1)} \subset \pi_{1}\left(W_{1}\right)^{(2)} \subset$ $\pi_{1}\left(W_{1}\right)_{\mathcal{M}}^{(2)}$. Let $\psi_{1}^{j}: \pi_{1}\left(M_{n-1}^{j}\right) \rightarrow \pi_{1}\left(W_{1}\right) \rightarrow \pi_{1}\left(W_{1}\right) / \pi_{1}\left(W_{1}\right)_{\mathcal{M}}^{(2)}$.

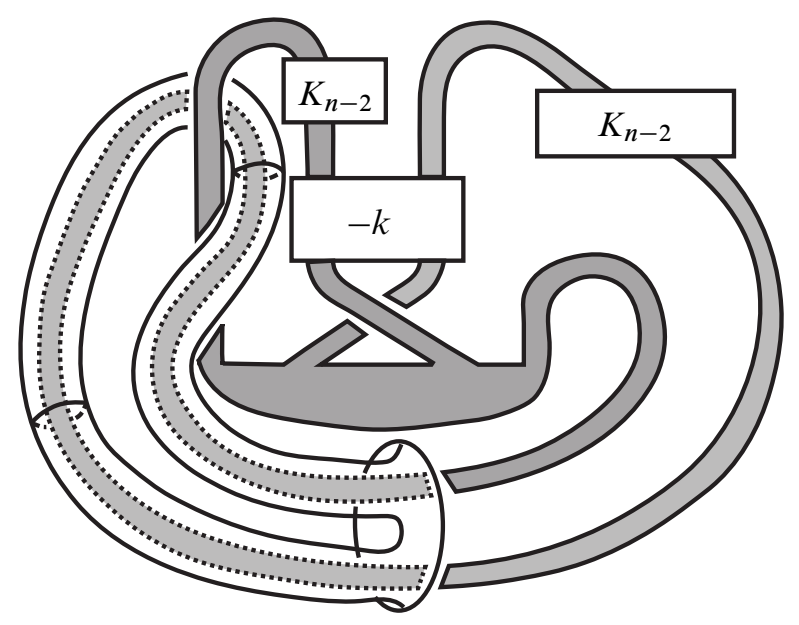

Figure 15: Seifert surfaces of $\widehat{L}_{n-1}$ 
Consider the composition of maps

$$
F \stackrel{k}{\rightarrow} \pi_{1}\left(M_{n-1}\right) \stackrel{\psi_{1}^{j}}{\longrightarrow} \Lambda_{1},
$$

where $F$ is a free group of rank 2 and $k$ is the map that sends the two generators of $F$ to the two meridians of $\widehat{L}_{n-1}^{j}$. One can observe that $k$ induces an isomorphism $H_{1}(F ; \mathbb{Q}) \stackrel{\cong}{\rightrightarrows} H_{1}\left(M_{n-1}^{j} ; \mathbb{Q}\right)$. Since $H_{2}\left(M_{n-1}^{j}, \mathbb{Q}\right)$ is spanned by surfaces whose fundamental groups are in the kernel of $\psi_{1}^{j}$, by Cochran and Harvey [7, Proposition 2.11] we know that $\operatorname{rank}_{\mathbb{Z} \Lambda_{1}} H_{1}\left(F, \mathbb{Z} \Lambda_{1}\right) \cong \operatorname{rank}_{\mathbb{Z} \Lambda_{1}} H_{1}\left(M_{n-1}, \mathbb{Z} \Lambda_{1}\right)$ and by $[11,2.12]$ we know that $\operatorname{rank}_{\mathbb{Z} \Lambda_{1}} H_{1}\left(F, \mathbb{Z} \Lambda_{1}\right)=1$.

Thus

$$
\psi_{1}^{j}: \pi_{1}\left(M_{n-1}^{j}\right) \rightarrow \Lambda_{1}
$$

is a nontrivial coefficient system with $\operatorname{rank}_{\mathbb{Z} \Lambda} H_{1}\left(M_{n-1}^{j}, \mathbb{Z} \Lambda_{1}\right)=\beta\left(M_{n-1}^{j}\right)-1$, and

$$
\psi_{1}^{j}: \pi_{1}\left(M_{\mathcal{R}_{j}^{n}}\right) \rightarrow \Lambda_{1}
$$

is a nontrivial coefficient system with $\operatorname{rank}_{\mathbb{Z} \Lambda} H_{1}\left(M_{\mathcal{R}_{j}^{n}}, \mathbb{Z} \Lambda\right)=\beta\left(M_{\mathcal{R}_{j}^{n}}\right)-1$ (the latter rank condition is automatically satisfied since $\mathcal{R}_{j}^{n}$ is a knot). By [10, Theorem 7.15], $P_{1}^{j} \cap T \mathcal{A}\left(M_{n-1}^{j}\right)$ is an isotropic submodule with respect to $\mathcal{B} \ell_{R_{1}^{M-1}}^{M_{n-1}^{j}}$, where $P_{1}^{j}=\operatorname{ker}\left(j_{1 *}^{j}\right)$. Since $\eta_{n-1}^{j, 1}$ and $\eta_{n-1}^{j, 2}$ generate $T \mathcal{A}\left(M_{n-1}^{j}\right)$, if both

$$
\eta_{n-1}^{j, 1}, \eta_{n-1}^{j, 2} \in P_{1}^{j} \cap T \mathcal{A}\left(M_{n-1}^{j}\right),
$$

it would follow that $\mathcal{B} \ell_{R_{1}} \widehat{L}_{n-1}^{j}$ is identically zero. We define $\mathcal{B} \ell_{R} \widehat{L}_{n-1}^{j}$ by the following commutative diagram, where PD is the map induced by Poincaré duality, $\kappa$ is the Kronecker evaluation map and $B$ is the map that arises from the Bockstein sequence of right $R_{1}$-modules. See [20] for a definition of $\mathcal{B} \ell_{R}^{M}$ for a general closed, connected, oriented 3-manifold $M$.

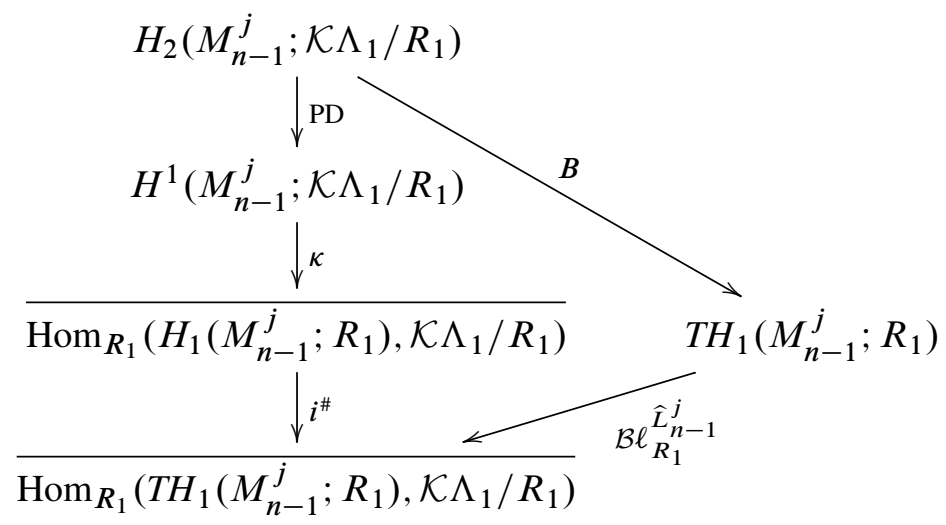


If $\mathcal{B} \ell_{R_{1}} \widehat{L}_{n-1}^{j}$ is identically zero, then the composition of vertical maps would be the trivial map. We will show that in fact this composition of maps is nontrivial and thus $\eta_{n-1}^{j, 1}$ or $\eta_{n-1}^{j, 2}$ must not lie in $P_{1}^{j}$ (which $\eta_{n-1}^{j, i}$ depends on $j$ ). It was shown in [11, Theorem 2.13] that the first map is an isomorphism. We will now show that the second map, the Kronecker evaluation map, is an isomorphism.

In [23], Levine defines a universal coefficient spectral sequence for the homology of the complement of a link with local coefficients. By analyzing this spectral sequence one can show that there is the short exact sequence

$$
\begin{aligned}
& 0 \rightarrow \operatorname{Ext}_{R_{1}}^{1}\left(H_{0}\left(M_{n-1} ; R_{1}\right), \mathcal{K} \Lambda_{1} / R_{1}\right) \rightarrow H^{1}\left(M_{n-1} ; \mathcal{K} \Lambda_{1} / R_{1}\right) \\
& \stackrel{\kappa}{\rightarrow}\left(\operatorname{Hom}_{R_{1}}\left(H_{1}\left(M_{n-1} ; R_{1}\right), \mathcal{K} \Lambda_{1} / R_{1}\right) \rightarrow \operatorname{Ext}_{R_{1}}^{2}\left(H_{0}\left(M_{n-1} ; R_{1}\right), \mathcal{K} \Lambda_{1} / R_{1}\right)\right) \rightarrow 0,
\end{aligned}
$$

where $\kappa$ is the Kronecker evaluation map. Therefore, we can show that

$$
H^{1}\left(M_{n-1}^{j} ; \mathcal{K} \Lambda_{1} / R_{1}\right) \cong \operatorname{Hom}_{R_{1}}\left(H_{1}\left(M_{n-1}^{j} ; R_{1}\right), \mathcal{K} \Lambda_{1} / R_{1}\right)
$$

if $\operatorname{Ext}_{R_{1}}^{1}\left(H_{0}\left(M_{n-1}^{j} ; R_{1}\right), \mathcal{K} \Lambda_{1} / R_{1}\right)$ and $\operatorname{Ext}_{R_{1}}^{2}\left(H_{0}\left(M_{n-1}^{j} ; R_{1}\right), \mathcal{K} \Lambda_{1} / R_{1}\right)$ are both the zero module.

Note that $H_{0}\left(M_{n-1}^{j} ; \mathbb{Q}\left[t_{1}^{ \pm 1}, t_{2}^{ \pm 1}\right]\right) \cong \mathbb{Q}\left[t_{1}^{ \pm 1}, t_{2}^{ \pm 1}\right] /\left\langle\left(t_{1}-1\right)\left(t_{2}-1\right)\right\rangle[26$, Exercise I2]. Thus

$$
H_{0}\left(M_{n-1}^{j} ; \mathbb{Q}\left[t_{1}^{ \pm 1}, t_{2}^{ \pm 1}\right]\right) \otimes_{\mathbb{Q}\left[t_{1}^{ \pm 1}, t_{2}^{ \pm 1}\right]} R_{1} \cong H_{0}\left(M_{n-1}^{j} ; R_{1}\right) \cong R_{1} /\left\langle\left(\eta_{n}^{j, 1}-1\right),\left(\eta_{n}^{j, 2}-1\right)\right\rangle .
$$

There is the following projective resolution,

$$
0 \rightarrow R_{1} \stackrel{\varphi}{\rightarrow} R_{1} \oplus R_{1} \stackrel{\psi}{\rightarrow} R_{1} \stackrel{\pi}{\rightarrow} \frac{R_{1}}{\left\langle\left(\eta_{n}^{j, 1}-1\right)\left(\eta_{n}^{j, 2}-1\right)\right\rangle} \rightarrow 0,
$$

where $\pi$ is the canonical quotient map. Let $\delta, \alpha, \beta$ and $\gamma$ all represent a generator for $R_{1}$; then $\psi$ can be defined as the map where $\alpha \mapsto\left(\eta_{n}^{j, 1}-1\right) \gamma$ and $\beta \mapsto\left(\eta_{n}^{j, 2}-1\right) \gamma$ and $\varphi$ can be defined as the map where $\delta \mapsto(\alpha-\beta)$. By applying the Hom functor, we obtain the following exact sequence:

$0 \leftarrow \operatorname{Hom}\left(R_{1}, \frac{\mathcal{K} \Lambda_{1}}{R_{1}}\right) \stackrel{\varphi^{*}}{\longleftarrow} \operatorname{Hom}\left(R_{1}, \frac{\mathcal{K} \Lambda_{1}}{R_{1}}\right) \oplus \operatorname{Hom}\left(R_{1}, \frac{\mathcal{K} \Lambda_{1}}{R_{1}}\right)$

$$
\stackrel{\psi^{*}}{\longleftarrow} \operatorname{Hom}\left(R_{1}, \frac{\mathcal{K} \Lambda_{1}}{R_{1}}\right)
$$

Let $x \in \mathcal{K} \Lambda_{1} / R_{1}$ be nontrivial. Note that $\gamma^{*}$ is defined by $\gamma \mapsto x, \alpha^{*}$ is defined by $\alpha \mapsto x$ and $\beta \mapsto 0, \beta^{*}$ is defined by $\beta \mapsto x$ and $\alpha \mapsto 0$, and $\delta^{*}$ is defined by $\delta \mapsto x$. We define $\psi^{*}$ as the map where $\gamma^{*} \mapsto \alpha^{*}\left(\eta_{n}^{j, 1}-1\right) \oplus \beta^{*}\left(\eta_{n}^{j, 2}-1\right)$ and $\varphi^{*}$ is defined as the map where $\alpha^{*} \mapsto \delta^{*}$ and $\beta^{*} \mapsto \delta(-1)=-\delta$, where $\alpha^{*}\left(\eta_{n}^{j, 1}-1\right) \oplus \beta^{*}\left(\eta_{n}^{j, 2}-1\right)$ is the 
homomorphism defined by $\alpha \mapsto\left(\eta_{n}^{j, 1}-1\right) x$ and $\beta \mapsto\left(\eta_{n}^{j, 2}-1\right) x$ and $-\delta$ is defined by $\delta \mapsto-x$. Note $\operatorname{Ext}_{R_{1}}^{1}\left(H_{0}\left(M_{n-1}^{j} ; R_{1}\right), \mathcal{K} \Lambda_{1} / R_{1}\right)=\operatorname{Hom}\left(R_{1}, \mathcal{K} \Lambda_{1} / R_{1}\right) / \operatorname{im}\left(\varphi^{*}\right) \equiv 0$. Also,

$$
\begin{aligned}
\operatorname{Ext}_{R_{1}}^{2}\left(H_{0}\left(M_{n-1}^{j} ; R_{1}\right) \mathcal{K} \Lambda_{1} / R_{1}\right)=\frac{\operatorname{ker}\left(\varphi^{*}\right)}{\operatorname{im}\left(\psi^{*}\right)} & =\frac{\left\langle\alpha^{*} \oplus \beta^{*}\right\rangle}{\left\langle\alpha^{*}\left(\eta_{n}^{j, 1}-1\right) \oplus \beta^{*}\left(\eta_{n}^{j, 2}-1\right)\right\rangle} \\
& =\frac{\left\langle\alpha^{*} \oplus \beta^{*}\right\rangle}{\left\langle\left(\left(\eta_{n}^{j, 1}-1\right) \oplus\left(\eta_{n}^{j, 2}-1\right)\right) \alpha^{*} \oplus \beta^{*}\right\rangle},
\end{aligned}
$$

and since $\left(\eta_{n}^{j, 1}-1\right) \oplus\left(\mu_{n}^{j, 2}-1\right) \in R_{1} \oplus R_{1}$,

$$
\operatorname{Ext}_{R_{1}}^{2}\left(H_{0}\left(M_{n-1}^{j} ; R_{1}\right) \mathcal{K} \Lambda_{1} / R_{1}\right) \cong \frac{\left\langle\alpha^{*} \oplus \beta^{*}\right\rangle}{\left\langle\alpha^{*} \oplus \beta^{*}\right\rangle} \equiv 0 .
$$

Therefore the Kronecker evaluation map is an isomorphism.

Now if we are able to show that $\operatorname{Hom}_{R_{1}}\left(T H_{1}\left(M_{n-1} ; R_{1}\right), \mathcal{K} \Lambda_{1} / R_{1}\right)$ is nontrivial, then $i^{\#}$ will be shown to be a nontrivial map and we will have shown that $\mathcal{B} \ell_{R_{1}}^{L_{n-1}^{j}}$ is not identically zero. First, we will have to demonstrate that $T H_{1}\left(M_{n-1}^{j} ; R_{1}\right)$ is nontrivial. Suppose that $T H_{1}\left(M_{n-1}^{j} ; R_{1}\right)$ is the zero module. By Theorem 4.9 we know that the map

$$
\frac{\mathbb{Q} \Lambda_{1}}{\mathfrak{m}_{k_{n-1}}^{i}\left(\eta_{n}^{j, 1}, \eta_{n}^{j, 2}\right) \mathbb{Q} \Lambda_{1}} \hookrightarrow \frac{\mathbb{Q} \Lambda_{1}}{\mathfrak{m}_{k_{n-1}}^{i}\left(\eta_{n}^{j, 1}, \eta_{n}^{j, 2}\right) \mathbb{Q} \Lambda_{1}} S_{\mathfrak{m}_{k_{n-1}}}^{-1} \cong \frac{R_{1}}{\mathfrak{m}_{k_{n-1}}^{i}\left(\eta_{n}^{j, 1}, \eta_{n}^{j, 2}\right) R_{1}}
$$

is a monomorphism. If $T H_{1}\left(M_{n-1}^{j} ; R_{1}\right)$ were the zero module, it would follow that

$$
\frac{\mathbb{Q} \Lambda_{1}}{\mathfrak{m}_{k_{n-1}}^{i}\left(\eta_{n}^{j, 1}, \eta_{n}^{j, 2}\right) \mathbb{Q} \Lambda_{1}} \equiv 0
$$

This would imply that $\mathfrak{m}_{k_{n-1}}^{i}\left(\eta_{n}^{j, 1}, \eta_{n}^{j, 2}\right)$ is a unit in $\mathbb{Q} \Lambda_{1}$.

Suppose that there is an $x \in \mathbb{Q} \Lambda_{1}$ such that $\mathfrak{m}_{k_{n-1}}^{i}\left(\eta_{n}^{j, 1}, \eta_{n}^{j, 2}\right) x=1$. Since $\mathbb{Q} \Lambda_{1}$ is a free

$$
\mathbb{Q}\left[t_{1}^{ \pm 1}, t_{2}^{ \pm 2}\right]=\mathbb{Q}\left[\eta_{n}^{j, 1^{ \pm 1}}, \eta_{n}^{j, 2}{ }^{ \pm 1}\right] \text {-module }
$$

we could decompose over the cosets of $\mathbb{Q}\left[\eta_{n}{ }^{j, 1^{ \pm 1}}, \eta_{n}{ }^{j, 2^{ \pm 1}}\right]$ to get

$$
\mathfrak{m}_{k_{n-1}}^{i}\left(\eta_{n}^{j, 1}, \eta_{n}^{j, 2}\right) x_{e}=1
$$

for some $x_{e} \in \mathbb{Q}\left[\eta_{n}{ }^{j, 1^{ \pm 1}}, \eta_{n}{ }^{j, 2^{ \pm 1}}\right]$. (Here we are using the fact that the $\eta_{n}^{j, 1}$ and $\eta_{n}^{j, 2}$ are of infinite order and linearly independent in $\mathbb{Q} \Lambda_{1}$.) This is not possible since $\mathfrak{m}_{k_{n-1}}^{i} \in \mathbb{Q}\left[t_{1}^{ \pm 1}, t_{2}^{ \pm 1}\right]$ is not a unit. Therefore

$$
T H_{1}\left(M_{n-1}^{j} ; R_{1}\right) \neq 0 .
$$


Recall that

$$
T H_{1}\left(M_{n-1}^{j} ; R_{1}\right) \cong \frac{R_{1}}{\mathfrak{m}_{k_{n-1}}^{1}\left(\eta_{n}^{j, 1}, \eta_{n}^{j, 2}\right) R_{1}} \oplus \frac{R_{1}}{\mathfrak{m}_{k_{n-1}}^{2}\left(\eta_{n}^{j, 1}, \eta_{n}^{j, 2}\right) R_{1}}
$$

Let $y_{1}$ and $y_{2}$ be the generators of the cyclic summands respectively. Consider the homomorphism $\tau: T H_{1}\left(M_{n-1}^{j} ; R_{1}\right) \rightarrow \mathcal{K} \Lambda_{1} / R_{1}$ where $y_{1} \mapsto 1 / m_{k_{n-1}}^{1}\left(\eta_{n}^{j, 1}, \eta_{n}^{j, 2}\right)$ and $y_{2} \mapsto 0$. This a nontrivial element of $\operatorname{Hom}_{R_{1}}\left(T H_{1}\left(M_{n-1}^{j} ; R_{1}\right) \mathcal{K} \Lambda_{1} / R_{1}\right)$ and therefore $\mathcal{B} \ell_{R_{1}}^{L_{n-1}^{j}}$ is not identically zero. Thus, $\eta_{n-1}^{j, 1}$ or $\eta_{n-1}^{j, 2}$ must not lie in $P_{1}^{j} \cap T \mathcal{A}\left(M_{n-1}^{j}\right)$. In conclusion, $\eta_{n-1}^{j, 1}$ or $\eta_{n-1}^{j, 2} \in \pi_{1}\left(M_{n-1}^{j}\right)$ (or both) is nontrivial in $\pi_{1}\left(W_{1}\right)^{(2)} / \pi_{1}\left(W_{1}\right)_{\mathcal{M}}^{(3)}$ for each $j$.

Stage 2 There is a cobordism $E_{n-1}^{j}$ with boundary $M_{n-1}^{j} \sqcup M_{\mathcal{R}_{j}^{n-1}} \sqcup M_{n-2}^{j, 1} \sqcup M_{n-2}^{j, 2}$. Let $W_{2}=\bigsqcup_{j} E_{n-1}^{j} \cup W_{1}$ where the union is along their common boundary $\bigsqcup_{j} M_{n-1}^{j}$. We wish to demonstrate that under the inclusion map $j_{2}^{j}: M_{n-2}^{j, i} \rightarrow W_{2}$,

$$
\begin{aligned}
& j_{2 *}^{j}\left(\pi_{1}\left(M_{n-2}^{j, i}\right)\right) \cong \mathbb{Z} \subset \pi_{1}\left(W_{2}\right)^{(2)} / \pi_{1}\left(W_{2}\right)_{\mathcal{M}}^{(3)} \text { or } \\
& j_{2 *}^{j}\left(\pi_{1}\left(M_{n-2}^{j, i}\right)\right) \equiv 0 \subset \pi_{1}\left(W_{2}\right)^{(2)} / \pi_{1}\left(W_{2}\right)_{\mathcal{M}}^{(3)},
\end{aligned}
$$

where, for each $j$, the first equation holds for $i=1$ or $i=2$ or both. Recall that $\pi_{1}\left(M_{n-2}^{j, i}\right)$ is normally generated by $\eta_{n-1}^{j, i}$ and, for each $j, \eta_{n-1}^{j, i}$ is nontrivial in $\pi_{1}\left(W_{1}\right)^{(2)} / \pi_{1}\left(W_{1}\right)_{\mathcal{M}}^{(3)}$ for $i=1$ or 2 or both. Therefore (11) can be demonstrated by showing

$$
\pi_{1}\left(W_{1}\right)^{(2)} / \pi_{1}\left(W_{1}\right)_{\mathcal{M}}^{(3)}=\pi_{1}\left(W_{2}\right)^{(2)} / \pi_{1}\left(W_{2}\right)_{\mathcal{M}}^{(3)}
$$

By Proposition 4.12 we need only show that

$$
\operatorname{ker}\left(\pi_{1}\left(W_{1}\right) \rightarrow \pi_{1}\left(W_{2}\right)\right) \subset \pi_{1}\left(W_{1}\right)_{\mathcal{M}}^{(3)} .
$$

The map

$$
\pi_{1}\left(W_{1}\right) \rightarrow \pi_{1}\left(W_{1} \cup_{\bigsqcup_{j} M_{n-1}^{j}} E_{n-1}^{j}\right)=\pi_{1}\left(W_{2}\right)
$$

is a surjection whose kernel is the normal closer of the longitudes, $\ell_{n-2}^{j, i}$, of $M_{n-2}^{j}$. Recall that $\eta_{n-1}^{j, i}$ is the meridian of the component with $\ell_{n-2}^{j, i}$ as its longitude. Thus, since $\eta_{n-1}^{j, i} \in \pi_{1}\left(W_{1}\right)^{(2)}, \ell_{n-2}^{j, i} \in \pi_{1}\left(W_{1}\right)^{(3)} \subset \pi_{1}\left(W_{1}\right)_{\mathcal{M}}^{(3)}$. Therefore, (12) is affirmed and (11) is asserted.

We will now demonstrate that $W_{2}$ is a $(n, \mathcal{M})$-bordism. We know that $W_{1}$ is a $(n, \mathcal{M})$-bordism. Since $W_{2}$ is created from $W_{1}$ by adding $\bigsqcup_{j} E_{n-1}^{j}$, where

$$
H_{2}\left(E_{n-1}^{j}\right) / H_{2}\left(\partial E_{n-1}^{j}\right)=0,
$$


a series of long exact sequences arguments shows that

$$
H_{2}\left(W_{1}\right) / H_{2}\left(\partial W_{1}\right)=H_{2}\left(W_{2}\right) / H_{2}\left(\partial W_{2}\right) .
$$

It then follows that the same surfaces used to show that $W_{1}$ is a $(n, \mathcal{M})$-bordism can be used to show that $W_{2}$ is a $(n, \mathcal{M})$-bordism since if $J$ is such a surface and $\pi_{1}(J) \subset \pi_{1}\left(W_{1}\right)_{\mathcal{M}}^{(n)}$ then $\pi_{1}(J) \subset \pi_{1}\left(W_{2}\right)_{\mathcal{M}}^{(n)}$ by the functorality of the derived series localized at $\mathcal{M}$.

We now proceed by reverse induction. Assume that for $2 \leq r \leq n-1$ that under the inclusion map $j_{r}^{j}: M_{n-r}^{j, i} \rightarrow W_{r}$,

$$
\begin{aligned}
& j_{r *}^{j}\left(\pi_{1}\left(M_{n-r}^{j, i}\right)\right) \cong \mathbb{Z} \subset \pi_{1}\left(W_{r}\right)^{(r)} / \pi_{1}\left(W_{r}\right)_{\mathcal{M}}^{(r+1)} \text { or } \\
& j_{r *}^{j}\left(\pi_{1}\left(M_{n-r}^{j, i}\right)\right) \equiv 0 \subset \pi_{1}\left(W_{r}\right)^{(r)} / \pi_{1}\left(W_{r}\right)_{\mathcal{M}}^{(r+1)},
\end{aligned}
$$

where, for a particular pair of $j$ and $r$, the first equation holds for $i=1$ or $i=2$ or both, and where $W_{r}$ is defined inductively by gluing copies of $E_{n-r}$, the cobordism with boundary equal to $M_{n-r} \sqcup M_{\mathcal{R}^{n-r-1}} \sqcup M_{n-r-1}$, along the copies of $M_{n-r}$. In addition, assume that $W_{r}$ is a $(n, \mathcal{M})$-bordism. Figure 16 demonstrates the construction of $W_{n}$.

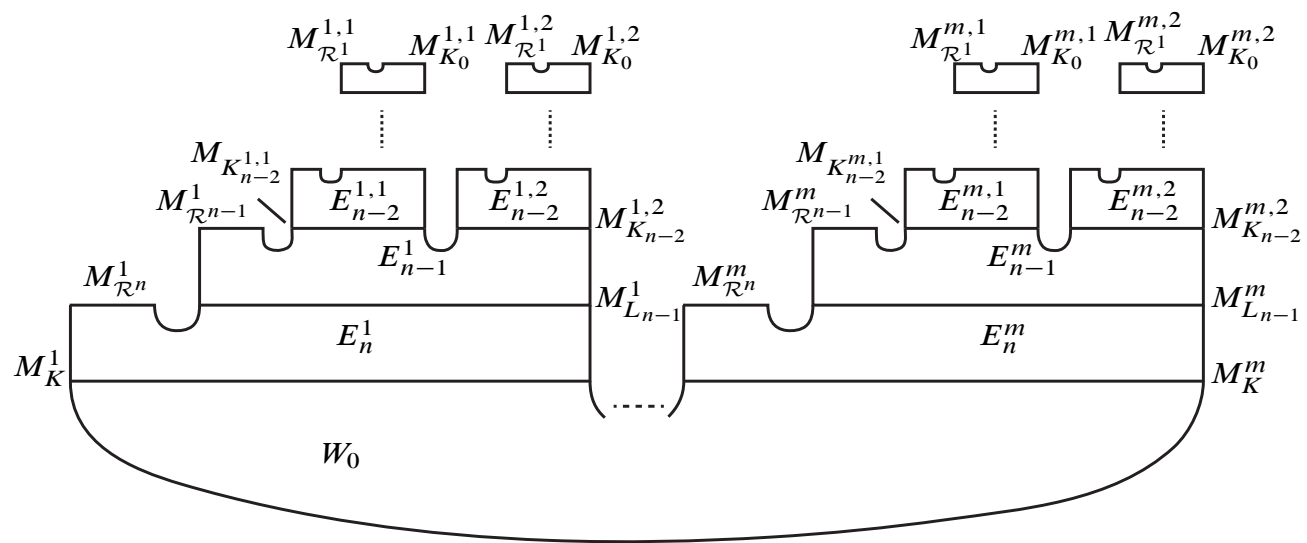

Figure 16: The construction of $W_{n}$

Given a particular $j$ and $r$, suppose the first equation of (13) holds for some $i$. We will show that $\eta_{n-r}^{j, i} \in \pi_{1}\left(M_{n-r}^{j, i}\right)$ is nontrivial in $\pi_{1}\left(W_{r}\right)^{(r+1)} / \pi_{1}\left(W_{r}\right)_{\mathcal{M}}^{(r+2)}$. Note that $\eta_{n-r}^{j, i} \in \pi_{1}\left(M_{n-r}^{j, i}\right)^{(1)}$. Since $j_{r *}^{j}\left(\pi_{1}\left(M_{n-r}^{j, i}\right)^{(1)}\right) \subset \pi_{1}\left(W_{r}\right)^{(r+1)}, \eta_{n-r}^{j, i} \in \pi_{1}\left(W_{r}\right)^{(r+1)}$.

Let $\Lambda_{r}=\pi_{1}\left(W_{r}\right) / \pi_{1}\left(W_{r}\right)_{\mathcal{M}}^{(r+2)}$ and let $\psi_{r}: \pi_{1}\left(W_{r}\right) \rightarrow \Lambda_{r}$ be the canonical surjection. Define $R_{r}=\left(\mathbb{Q} \Lambda_{r}\right) S_{p_{n-r}}^{-1}$. Note

$$
H_{1}\left(W_{r} ; R_{r}\right) \cong \pi_{1}\left(W_{r}\right)_{\mathcal{M}}^{(r+1)} /\left[\pi_{1}\left(W_{r}\right)_{\mathcal{M}}^{(r+1)}, \pi_{1}\left(W_{r}\right)_{\mathcal{M}}^{(r+1)}\right] \otimes_{\mathbb{Z} \Lambda_{r}} R_{r} .
$$


Consider the following commutative diagram:

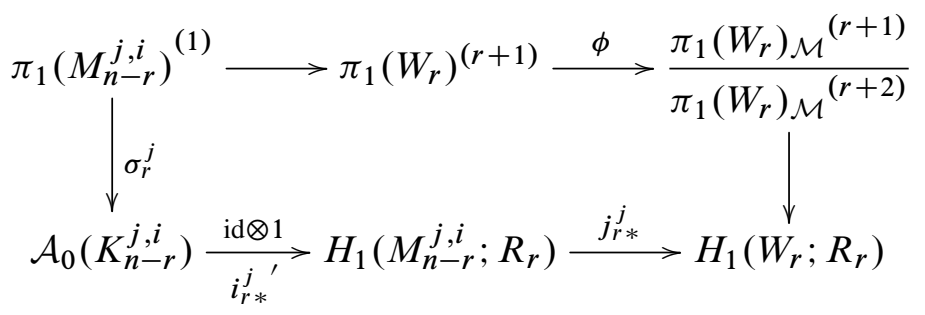

If $\eta_{n-r}^{j, i}$ is in the kernel of the top row, then $\eta_{n-r}^{j, i} \in \mathcal{A}_{0}\left(K_{n-r}^{j, i}\right)$ must be in the kernel of the bottom row. If this were true then $\eta_{n, i}^{j, i} \otimes 1$ would be in the kernel of $j_{r *}^{j}$. Since by assumption $\psi_{r}$ restricted to $\pi_{1}\left(M_{n-r}^{j, i}\right)$ is nontrivial and factors through the abelianization, by [10, Theorem 7.15]

$$
\operatorname{ker} j_{r *}^{j}=P_{r}^{j} \subset P_{r}^{j \perp}
$$

is an isotropic submodule with respect to the Blanchfield form on $H_{1}\left(M_{n-r}^{j, i} ; R_{r}\right)$. Therefore, $\eta_{n-r}^{j, i}$ is in the kernel of the bottom row if $\eta_{n-r}^{j, i} \otimes 1$ is in $P_{r}^{j}$. If $\eta_{n-r}^{j, i} \otimes 1$ were in $P_{r}^{j}$, then $\mathcal{B} \ell_{R_{r}} K_{n-i}^{j, i}$ would be identically zero. But by [10, Lemma 7.16] we know that $\mathcal{B} \ell_{R_{1}} K_{n-r}^{j, i}$ is nonsingular. This is only possible if $H_{1}\left(M_{n-r}^{j, i} ; R_{r}\right)$ is the zero module. Theorem 4.9 informs us that

$$
\frac{\mathbb{Q} \Lambda_{r}}{\mathfrak{p}_{k_{n-r}}\left(\eta_{n-r+1}^{j, i}\right) \mathbb{Q} \Lambda_{r}} \hookrightarrow \frac{\mathbb{Q} \Lambda_{r}}{\mathfrak{p}_{k_{n-r}}\left(\eta_{n-r+1}^{j, i}\right) \mathbb{Q} \Lambda_{r}} S_{\mathfrak{p}_{k_{n-r}}}^{-1} \cong \frac{R_{r}}{\mathfrak{p}_{k_{n-r}}\left(\eta_{n-r+1}^{j, i}\right) R_{r}}
$$

is a monomorphism. Therefore, if $H_{1}\left(M_{n-r}^{j, i} ; R_{r}\right)$ were the zero module, it would follow that

$$
\frac{\mathbb{Q} \Lambda_{r}}{\mathfrak{p}_{k_{n-r}}\left(\eta_{n-r+1}^{j, i}\right) \mathbb{Q} \Lambda_{r}} \equiv 0
$$

This would imply that $\mathfrak{p}_{k_{n-r}}\left(\eta_{n-r+1}^{j, i}\right)$ is a unit in $\mathbb{Q} \Lambda_{r}$. Suppose that there is an $x \in \mathbb{Q} \Lambda_{r}$ such that $\mathfrak{p}_{k_{n-r}}\left(\eta_{n-r+1}^{j, i}\right) x=1$. Since $\mathbb{Q} \Lambda_{r}$ is a free $\mathbb{Q}\left[t, t^{-1}\right]=$ $\mathbb{Q}\left[\eta_{n-r+1}^{j, i}, \eta_{n-r+1}^{j, i}\right]$-module, we could decompose over the cosets of

$$
\mathbb{Q}\left[\eta_{n-r+1}^{j, i}, \eta_{n-r+1}^{j, i^{-1}}\right]
$$

to get $\mathfrak{p}_{k_{n-r}}\left(\eta_{n-r+1}^{j, i}\right) x_{e}=1$ for some $x_{e} \in \mathbb{Q}\left[\eta_{n-r+1}^{j, i}, \eta_{n-r+1}^{j, i}{ }^{-1}\right]$. (Here we are using the fact that the $\eta_{n-r}^{j, i}$ is of infinite order and linearly independent in $\mathbb{Q} \Lambda_{r}$.) This is not possible since $\mathfrak{p}_{k_{n-r}} \in \mathbb{Q}\left[t, t^{-1}\right]$ is not a unit. Therefore

$$
H_{1}\left(M_{n-r}^{j, i} ; R_{r}\right) \neq 0 .
$$

In conclusion, $\eta_{n-r}^{j, i} \otimes 1$ is not in $P_{r}^{j}$ and thus $\eta_{n-r}^{j, i} \in \pi_{1}\left(M_{n-r}^{j, i}\right)$ is nontrivial in $\pi_{1}\left(W_{r}\right)^{(r+1)} / \pi_{1}\left(W_{r}\right)_{\mathcal{M}}^{(r+2)}$. 
Given a particular $j$ and $r$, suppose, alternatively, the second equation of (13) holds for some $i$. Since $j_{r *}^{j}\left(\pi_{1}\left(M_{n-r}^{j, i}\right)\right) \subset \pi_{1}\left(W_{r}\right)_{\mathcal{M}}^{(r+1)}$ and $\eta_{n-r}^{j, i} \in \pi_{1}\left(M_{n-r}^{j, i}\right)^{(1)}, \eta_{n-r}^{j, i} \in$ $\pi_{1}\left(W_{r}\right)_{\mathcal{M}}^{(r+2)}$. Thus $\eta_{n-r}^{j, i} \in \pi_{1}\left(M_{n-r}^{j, i}\right)$ is trivial in $\pi_{1}\left(W_{r}\right)^{(r+1)} / \pi_{1}\left(W_{r}\right)_{\mathcal{M}}^{(r+2)}$.

Thus, for each pair of $j$ and $r, \eta_{n-r-1}^{j, i} \in \pi_{1}\left(M_{n-r-1}^{j, i}\right)$ is nontrivial in

$$
\pi_{1}\left(W_{r}\right)^{(r+1)} / \pi_{1}\left(W_{r}\right)_{\mathcal{M}}^{(r+2)}
$$

for $i=1$ or $i=2$ or both.

Stage $r+1$ Next we demonstrate that under the inclusion map $j_{r+1}^{j, i}: M_{n-r-1}^{j, i} \rightarrow W_{r+1}$,

$$
\begin{aligned}
& j_{r+1 *}^{j, i}\left(\pi_{1}\left(M_{n-r-1}^{j, i}\right)\right) \cong \mathbb{Z} \subset \pi_{1}\left(W_{r+1}\right)^{(r+1)} / \pi_{1}\left(W_{r+1}\right)_{\mathcal{M}}^{(r+2)} \text { or } \\
& j_{r+1 *}^{j, i}\left(\pi_{1}\left(M_{n-r-1}^{j, i}\right)\right) \equiv 0 \subset \pi_{1}\left(W_{r+1}\right)^{(r+1)} / \pi_{1}\left(W_{r+1}\right)_{\mathcal{M}}^{(r+2)}
\end{aligned},
$$

where, for a particular pair of $j$ and $r$, the first equation holds for $i=1$ or $i=2$ or both. Note (14) can be demonstrated by showing

$$
\pi_{1}\left(W_{r}\right)^{(r+1)} / \pi_{1}\left(W_{r}\right)_{\mathcal{M}}^{(r+2)}=\pi_{1}\left(W_{r+1}\right)^{(r+1)} / \pi_{1}\left(W_{r+1}\right)_{\mathcal{M}}^{(r+2)} .
$$

Proposition 4.12 implies that we only need to show that

$$
\operatorname{ker}\left(\pi_{1}\left(W_{r}\right) \rightarrow \pi_{1}\left(W_{r+1}\right)\right) \subset \pi_{1}\left(W_{r}\right)_{\mathcal{M}}^{(r+2)} .
$$

The map $\pi_{1}\left(W_{r}\right) \rightarrow \pi_{1}\left(W_{r} \cup_{M_{n-r}^{j, i}} \bigsqcup_{i} E_{n-r}^{j, i}\right)=\pi_{1}\left(W_{r+1}\right)$ is a surjection whose kernel is the normal closer of the longitudes, $\ell_{n-r-1}^{j, i}$, of $M_{n-r-1}^{j, i}$. Recall that $\eta_{n-r}^{j, i}$ is the meridian of $M_{n-r-1}^{j, i}$. Thus, since $\eta_{n-r}^{j, i} \in \pi_{1}\left(W_{r}\right)^{(r+1)}, \ell_{n-r-1}^{j-r, 1} \in \pi_{1}\left(W_{r}\right)^{(r+2)} \subset$ $\pi_{1}\left(W_{r}\right)_{\mathcal{M}}^{(r+1)}$. Thus, (15) is affirmed and (14) is asserted.

In particular, it has been shown that for $j_{n *}^{j, i}: M_{0}^{j, i} \rightarrow W_{n}$,

$$
\begin{aligned}
& j_{n *}^{j, i}\left(\pi_{1}\left(M_{0}^{j, i}\right)\right) \cong \mathbb{Z} \subset \pi_{1}\left(W_{n}\right)^{(n)} / \pi_{1}\left(W_{n}\right)_{\mathcal{M}}^{(n+1)} \text { or } \\
& j_{n *}^{j, i}\left(\pi_{1}\left(M_{0}^{j, i}\right)\right) \equiv 0 \subset \pi_{1}\left(W_{n}\right)^{(n)} / \pi_{1}\left(W_{n}\right)_{\mathcal{M}}^{(n+1)},
\end{aligned}
$$

where, for each $j$, the first equation holds for $i=1$ or $i=2$ or both.

We will now demonstrate that $W_{r+1}$ is a $(n, \mathcal{M})$-bordism. We know that $W_{r}$ is a $(n, \mathcal{M})$-bordism. Since $W_{r+1}$ is created from $W_{r}$ by adding copies of $E_{n-r}$ where $H_{2}\left(E_{n-r}^{j, i}\right) / H_{2}\left(\partial E_{n-r}^{j, i}\right)=0$, a series of long exact sequence arguments analogous to those used in Step 1 shows that

$$
H_{2}\left(W_{r}\right) / H_{2}\left(\partial W_{r}\right)=H_{2}\left(W_{r_{1}}\right) / H_{2}\left(\partial W_{r+1}\right) .
$$

It then follows that the same surfaces used to show that $W_{r}$ is a $(n, \mathcal{M})$-bordism can be used to show that $W_{r+1}$ is a $(n, \mathcal{M})$-bordism since if $J$ is such a surface and 
$\pi_{1}(J) \subset \pi_{1}\left(W_{r}\right)_{\mathcal{M}}^{(n)}$, then $\pi_{1}(J) \subset \pi_{1}\left(W_{r+1}\right)_{\mathcal{M}}^{(n)}$ by the functorality of the derived series localized at $\mathcal{M}$.

To conclude the proof, we will calculate $\rho\left(\partial W_{n}, \phi\right)=\sigma^{2}\left(W_{n}, \phi\right)-\sigma\left(W_{n}\right)$, where $\phi: \pi_{1}\left(W_{n}\right) \rightarrow \pi_{1}\left(W_{n}\right) / \pi_{1}\left(W_{n}\right)_{\mathcal{M}}^{(n+1)}$. Recall that by [11, Theorem 4.2], $\sigma^{2}(V, \phi)-$ $\sigma(V)=0$. By Lemma 2.2, $\sigma\left(E_{r}^{j, i}\right)=0$ for all $i, j$ and $r$, and by Lemma 2.3, $\sigma^{2}\left(E_{r}^{j, i}, \phi\right)=0$ for all $r \neq n$. Thus by [10, Proposition 5.1, Property (5)], $\sigma^{2}\left(W_{n}, \phi\right)-$ $\sigma\left(W_{n}\right)=\sum_{j} \sigma^{2}\left(E_{n}^{j}, \phi\right)$. Therefore

$$
\begin{aligned}
\sum_{j} \sigma^{2}\left(E_{n}^{j}, \phi\right)=\sum_{j}\left(\rho\left(M_{\mathcal{R}_{j}^{n}}, \phi\right)+\rho\left(M_{\hat{\mathcal{R}}_{j}^{n-1}}, \phi\right)+\rho\left(M_{\mathcal{R}_{j}^{n-2}}^{1}, \phi\right)+\rho\left(M_{\mathcal{R}_{j}^{n-2}}^{2}, \phi\right)\right. \\
\left.+\cdots+\rho\left(M_{\mathcal{R}_{j}^{1}}^{1}, \phi\right)+\rho\left(M_{\mathcal{R}_{j}^{1}}^{2}, \phi\right)+\rho\left(M_{K_{0}}^{j, 1}, \phi\right)+\rho\left(M_{K_{0}}^{j, 2}, \phi\right)\right) .
\end{aligned}
$$

Thus

$$
\begin{aligned}
& \sum_{j}\left(-\rho\left(M_{K_{0}}^{j, 1}, \phi\right)\right.\left.-\rho\left(M_{K_{0}}^{j, 2}, \phi\right)\right) \\
&=\sum_{j}\left(\rho\left(M_{\mathcal{R}_{j}^{n}}, \phi\right)+\rho\left(M_{\hat{\mathcal{R}}_{j}^{n-1}}, \phi\right)+\rho\left(M_{\mathcal{R}_{j}^{n-2}}^{1}, \phi\right)+\rho\left(M_{\mathcal{R}_{j}^{n-2}}^{2}, \phi\right)\right. \\
&\left.+\cdots+\rho\left(M_{\mathcal{R}_{j}^{1}}^{1}, \phi\right)+\rho\left(M_{\mathcal{R}_{j}^{1}}^{2}, \phi\right)-\sum_{j} \sigma^{2}\left(E_{n}^{j}, \phi\right)\right) .
\end{aligned}
$$

By [10, Lemma 5.1, parts (1)-(3)], and (16), we know $\rho\left(M_{K_{0}}^{j, i}, \phi\right)=\rho_{0}\left(M_{K_{0}}\right)$ or 0 and we are guaranteed at least $m$ incidents where $\rho\left(M_{K_{0}, i}^{j, \phi}\right)=\rho_{0}\left(M_{K_{0}}\right)$. Thus,

$$
\begin{aligned}
-k \rho_{0}\left(M_{K_{0}}\right)=\sum_{j}\left(\rho\left(M_{\mathcal{R}_{j}^{n}}, \phi\right)+\right. & \rho\left(M_{\widehat{\mathcal{R}}_{j}^{n-1}}, \phi\right)+\rho\left(M_{\mathcal{R}_{j}^{n-2}}^{1}, \phi\right)+\rho\left(M_{\mathcal{R}_{j}^{n-2}}^{2}, \phi\right) \\
& \left.+\cdots+\rho\left(M_{\mathcal{R}_{j}^{1}}^{1}, \phi\right)+\rho\left(M_{\mathcal{R}_{j}^{1}}^{2}, \phi\right)-\sum_{j} \sigma^{2}\left(E_{n}^{j}, \phi\right)\right),
\end{aligned}
$$

where $m \leq k$ and $k \in \mathbb{Z}^{+}$. We can then conclude that

$$
\begin{aligned}
\left|k \rho_{0}\left(M_{K_{0}}\right)\right|=\mid \sum_{j}\left(\rho\left(M_{\mathcal{R}_{j}^{n}}, \phi\right)\right. & +\rho\left(M_{\hat{\mathcal{R}}_{j}^{n-1}}, \phi\right)+\rho\left(M_{\mathcal{R}_{j}^{n-2}}^{1}, \phi\right)+\rho\left(M_{\mathcal{R}_{j}^{n-2}}^{2}, \phi\right) \\
& \left.+\cdots+\rho\left(M_{\mathcal{R}_{j}^{1}}^{1}, \phi\right)+\rho\left(M_{\mathcal{R}_{j}^{1}}^{2}, \phi\right)-\sum_{j} \sigma^{2}\left(E_{n}^{j}, \phi\right)\right) \mid .
\end{aligned}
$$

Therefore,

$$
\begin{array}{r}
\left|k \rho_{0}\left(M_{K_{0}}\right)\right| \leq \sum_{j}\left(\left|\rho\left(M_{\mathcal{R}_{j}^{n}}, \phi\right)\right|+\left|\rho\left(M_{\hat{\mathcal{R}}_{j}^{n-1}}, \phi\right)\right|+\left|\rho\left(M_{\mathcal{R}_{j}^{n-2}}^{1}, \phi\right)\right|+\left|\rho\left(M_{\mathcal{R}_{j}^{n-2}}^{2}, \phi\right)\right|\right. \\
\left.+\cdots+\left|\rho\left(M_{\mathcal{R}_{j}^{1}}^{1}, \phi\right)\right|+\left|\rho\left(M_{\mathcal{R}_{j}^{1}}^{2}, \phi\right)\right|+\sum_{j}\left|\sigma^{2}\left(E_{n}^{j}, \phi\right)\right|\right) .
\end{array}
$$


We can then infer that

$$
m\left|\rho_{0}\left(M_{K_{0}}\right)\right| \leq m\left(C_{\mathcal{R}^{n}}+C_{\widehat{\mathcal{R}}^{n-1}}+C_{\mathcal{R}^{n-2}}+C_{\mathcal{R}^{n-2}}+\cdots+C_{\mathcal{R}^{1}}+C_{\mathcal{R}^{1}}+3\right) .
$$

By [4, Lemma 2.7], $\left|\sigma^{2}\left(E_{n}, \phi\right)\right| \leq \beta_{2}\left(E_{n}\right)=\beta_{2}\left(M_{\mathcal{R}^{n}}\right)+\beta_{2}\left(M_{L_{n-1}}\right)=1+2=3$, where the first equality is by part (4) of Lemma 2.1. (Note $H_{2}\left(M_{\mathcal{R}^{n}}\right)=\mathbb{Z}$ and $H_{2}\left(M_{L_{n-1}}\right)=\mathbb{Z} \oplus \mathbb{Z}$, where each $\mathbb{Z}$ summand is generated by a capped off Seifert surface for a component of $\mathcal{R}_{n}$ and $L_{n-1}$ respectively.)

Recall that $K_{0}$ was chosen so that $\left|\rho_{0}\left(M_{K_{0}}\right)\right|>C_{\mathcal{R}^{n}}+C_{\widehat{\mathcal{R}}^{n-1}}+C_{\mathcal{R}^{n-2}}+C_{\mathcal{R}^{n-2}}+$ $\cdots+C_{\mathcal{R}^{1}}+C_{\mathcal{R}^{1}}+3$. This is a contradiction. Thus $K$ cannot bound a $(n .5, \mathcal{M})-$ solution.

Theorem 6.4 For $n \geq 2$, there exists a knot, $K_{n}$, of infinite order in $\mathcal{F}_{n} / \mathcal{F}_{n .5}$ such that no multiple of $K_{n}$ is concordant to any knots created by two or more applications of nontrivial knot doubling operators to knots in $\mathcal{F}_{0}$. In particular,

$$
m K_{n} \notin \bigoplus_{\mathbb{P}_{n}} \mathbb{Z}^{\infty} \subset \mathcal{F}_{n} / \mathcal{F}_{n .5}
$$

for all $m \in \mathbb{Z}^{+}$. Thus, $m K_{n}$ is distinct from all $C H L$ knots for all $n$ and $m \in \mathbb{Z}^{+}$.

Proof By Theorem 6.2, for any $n$ there exists a knot $K_{n} \in \mathcal{F}_{n} / \mathcal{F}_{n .5}$ of infinite order in $\mathcal{F}_{n} / \mathcal{F}_{n .5}^{\mathcal{M}}$ and by Theorem 5.2 and Lemma 4.5 we know that for any knot, $J$, created by two or more applications of nontrivial knot doubling operators to a knot in $\mathcal{F}_{0}, J$ is trivial in $\mathcal{F}_{n} / \mathcal{F}_{n .5}^{\mathcal{M}}$. Since $\left\{\mathcal{F}_{n}^{(\mathcal{M})}\right\}$ is a filtration of the concordance group this implies that $K_{n}^{m}$ is not concordant to $J$ for all $m \neq 0$.

\section{References}

[1] A J Casson, CM Gordon, On slice knots in dimension three, from: "Algebraic and geometric topology, Part 2", (R J Milgram, editor), Proc. Sympos. Pure Math. 32, Amer. Math. Soc. (1978) 39-53 MR520521

[2] A J Casson, C M Gordon, Cobordism of classical knots, from: "Á la recherche de la topologie perdue", (L Guillou, A Marin, editors), Progr. Math. 62, Birkhäuser, Boston, MA (1986) 181-199 MR900252

[3] J C Cha, The structure of the rational concordance group of knots, Mem. Amer. Math. Soc. 885, Amer. Math. Soc. (2007) MR2343079

[4] J C Cha, Topological minimal genus and $L^{2}$-signatures, Algebr. Geom. Topol. 8 (2008) 885-909 MR2443100

[5] T D Cochran, Noncommutative knot theory, Algebr. Geom. Topol. 4 (2004) 347-398 MR2077670 
[6] T D Cochran, S Friedl, P Teichner, New constructions of slice links, Comment. Math. Helv. 84 (2009) 617-638 MR2507256

[7] T D Cochran, S Harvey, Homology and derived series of groups, II: Dwyer's theorem, Geom. Topol. 12 (2008) 199-232 MR2377249

[8] T D Cochran, S Harvey, C Leidy, Link concordance and generalized doubling operators, Algebr. Geom. Topol. 8 (2008) 1593-1646 MR2443256

[9] T D Cochran, S Harvey, C Leidy, Knot concordance and higher-order Blanchfield duality, Geom. Topol. 13 (2009) 1419-1482 MR2496049

[10] T D Cochran, S Harvey, C Leidy, Primary decomposition and the fractal nature of knot concordance, Math. Ann. 351 (2011) 443-508 MR2836668

[11] T D Cochran, KE Orr, P Teichner, Knot concordance, Whitney towers and $L^{2}-$ signatures, Ann. of Math. 157 (2003) 433-519 MR1973052

[12] T D Cochran, K E Orr, P Teichner, Structure in the classical knot concordance group, Comment. Math. Helv. 79 (2004) 105-123 MR2031301

[13] T D Cochran, P Teichner, Knot concordance and von Neumann $\rho$-invariants, Duke Math. J. 137 (2007) 337-379 MR2309149

[14] R H Fox, J W Milnor, Singularities of 2-spheres in 4-space and cobordism of knots, Osaka J. Math. 3 (1966) 257-267 MR0211392

[15] J A Hillman, Alexander ideals of links, Lecture Notes in Mathematics 895, Springer, Berlin (1981) MR653808

[16] B J Jiang, A simple proof that the concordance group of algebraically slice knots is infinitely generated, Proc. Amer. Math. Soc. 83 (1981) 189-192 MR620010

[17] M A Kervaire, J W Milnor, On 2-spheres in 4-manifolds, Proc. Nat. Acad. Sci. U.S.A. 47 (1961) 1651-1657 MR0133134

[18] S-G Kim, Polynomial splittings of Casson-Gordon invariants, Math. Proc. Cambridge Philos. Soc. 138 (2005) 59-78 MR2127228

[19] S-G Kim, T Kim, Polynomial splittings of metabelian von Neumann rho-invariants of knots, Proc. Amer. Math. Soc. 136 (2008) 4079-4087 MR2425750

[20] C Leidy, Higher-order linking forms, PhD thesis, Rice University (2004) arXiv: $1204.5167 \mathrm{v} 1$

[21] C Leidy, Higher-order linking forms for knots, Comment. Math. Helv. 81 (2006) 755-781 MR2271220

[22] J Levine, Knot cobordism groups in codimension two, Comment. Math. Helv. 44 (1969) 229-244 MR0246314

[23] J Levine, Knot modules, I, Trans. Amer. Math. Soc. 229 (1977) 1-50 MR0461518

[24] J Levine, Signature invariants of homology bordism with applications to links, from: “Knots 90”, (A Kawauchi, editor), de Gruyter, Berlin (1992) 395-406 MR1177436 
[25] D S Passman, The algebraic structure of group rings, Robert E Krieger Publishing Co., Melbourne, FL (1985) MR798076

[26] D Rolfsen, Knots and links, Mathematics Lecture Series 7, Publish or Perish, Houston, TX (1990) MR1277811

[27] N W Stoltzfus, Unraveling the integral knot concordance group, Mem. Amer. Math. Soc. 192, Amer. Math. Soc. (1977) MR0467764

Mathematics and Computer Science Department, Rhode Island College 600 Mount Pleasant Avenue, Providence, RI 02908-1991, USA jburke@ric.edu

Received: 9 August 2011 Revised: 15 August 2013 